
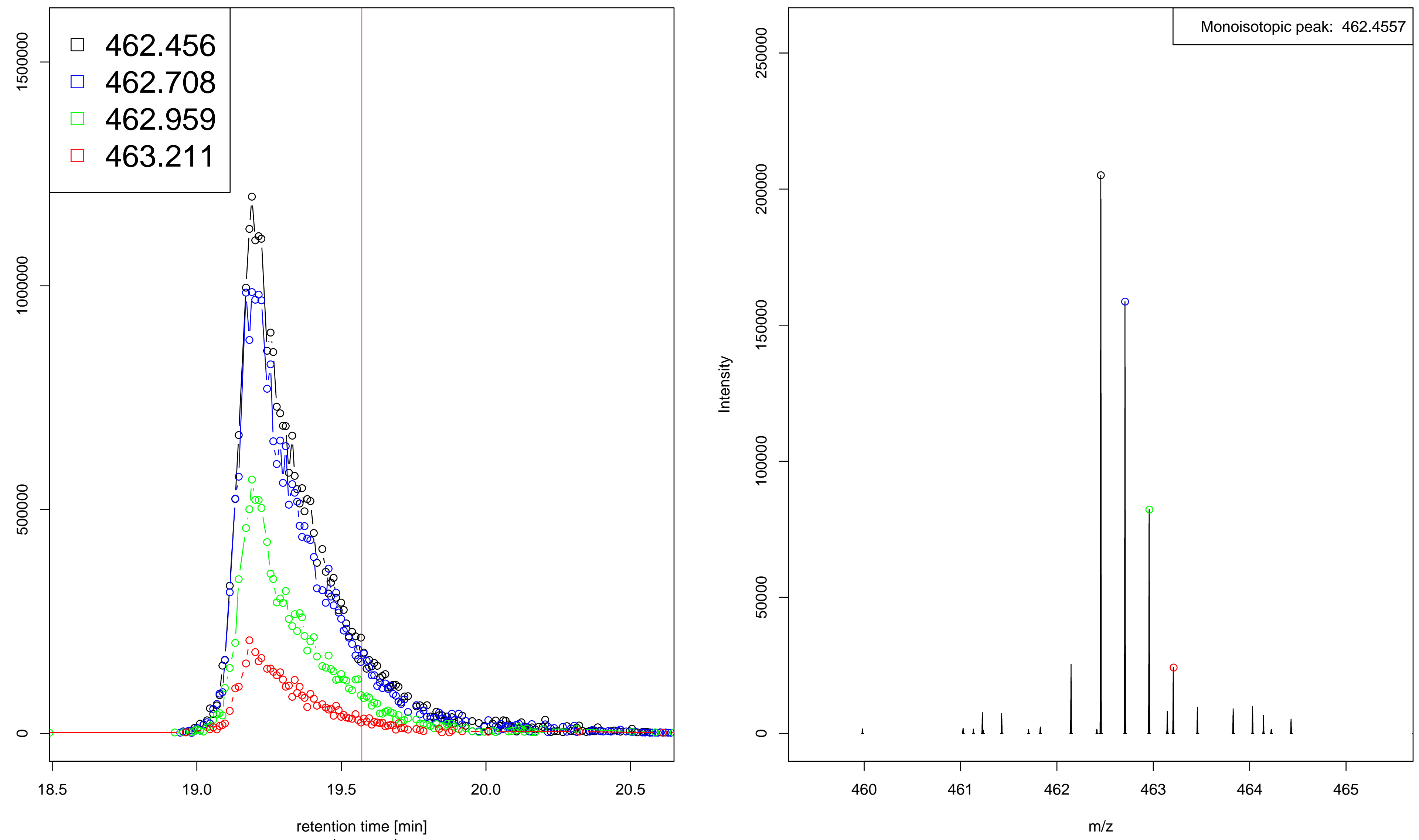

charge $=4$ 
XIC of MS1

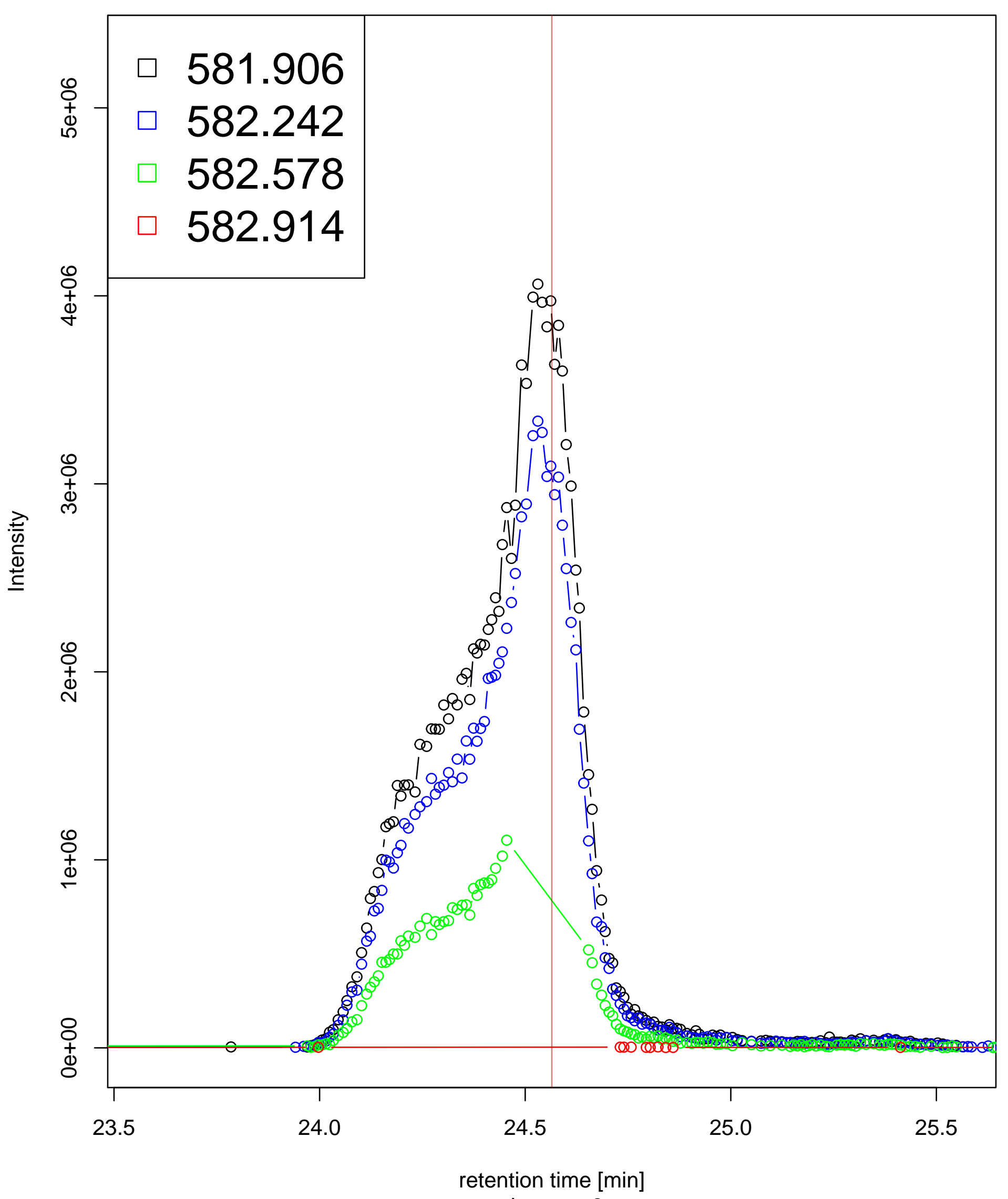

MS1 of ScanNo:3304

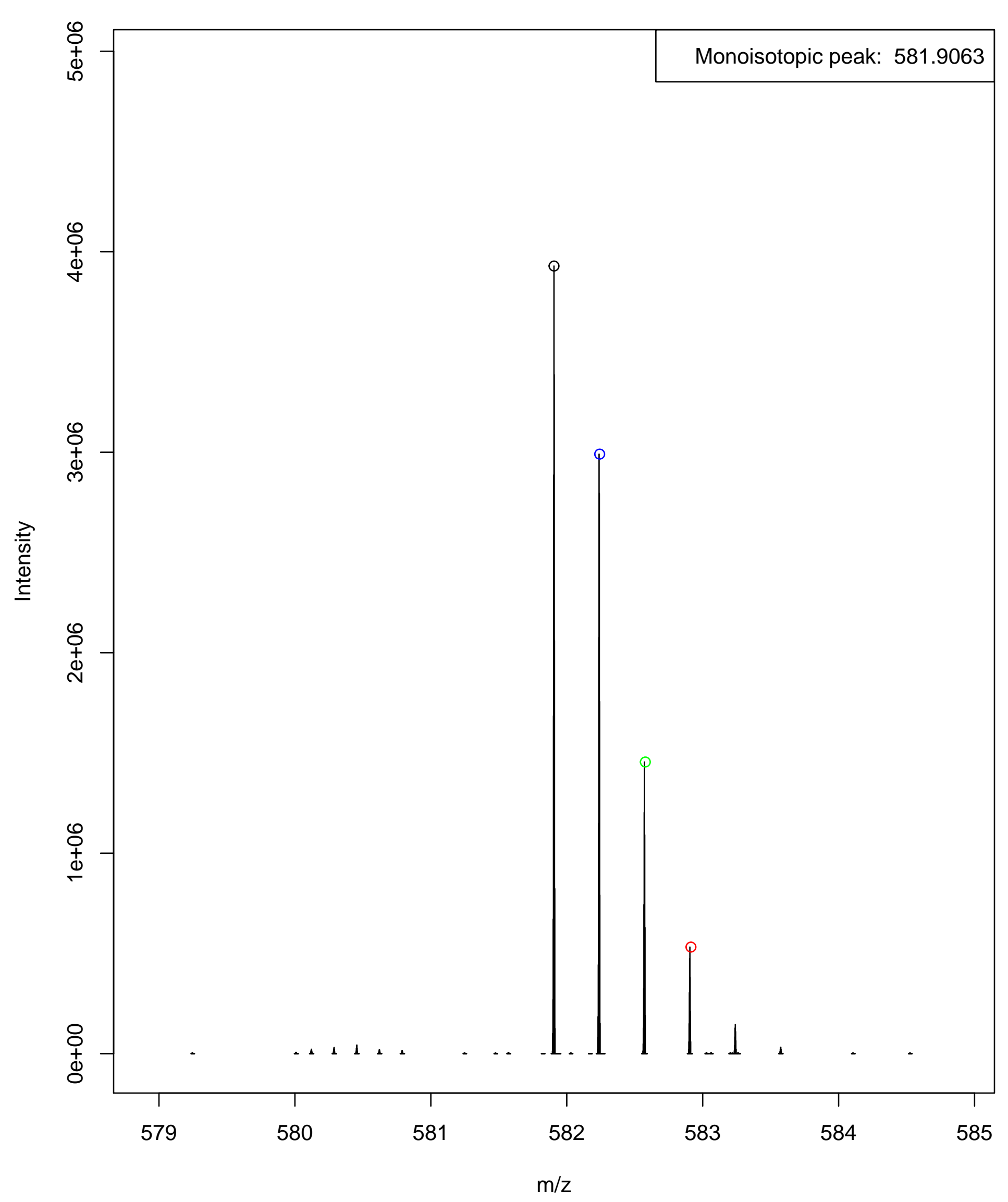


XIC of MS1

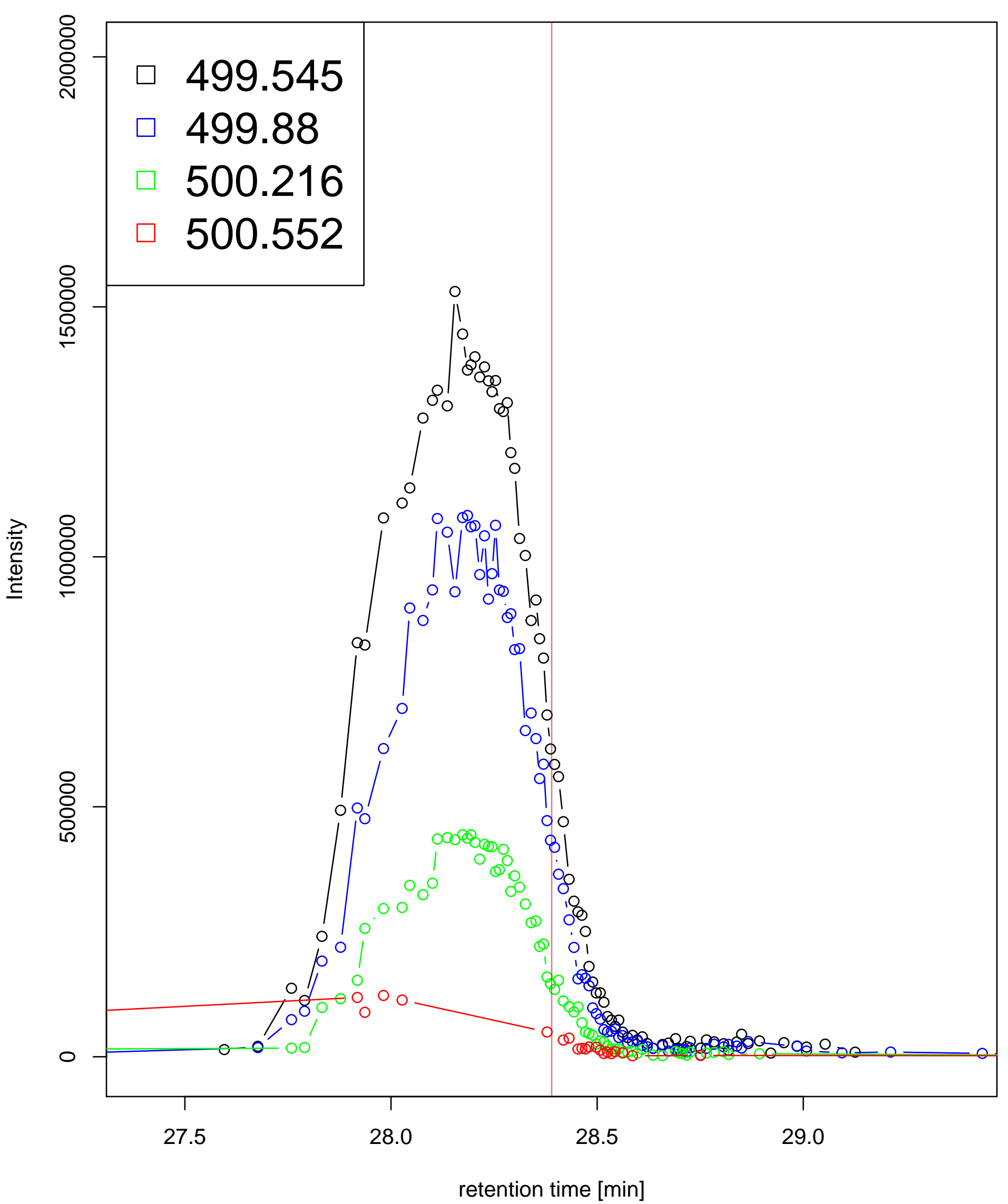

MS1 of ScanNo:4056

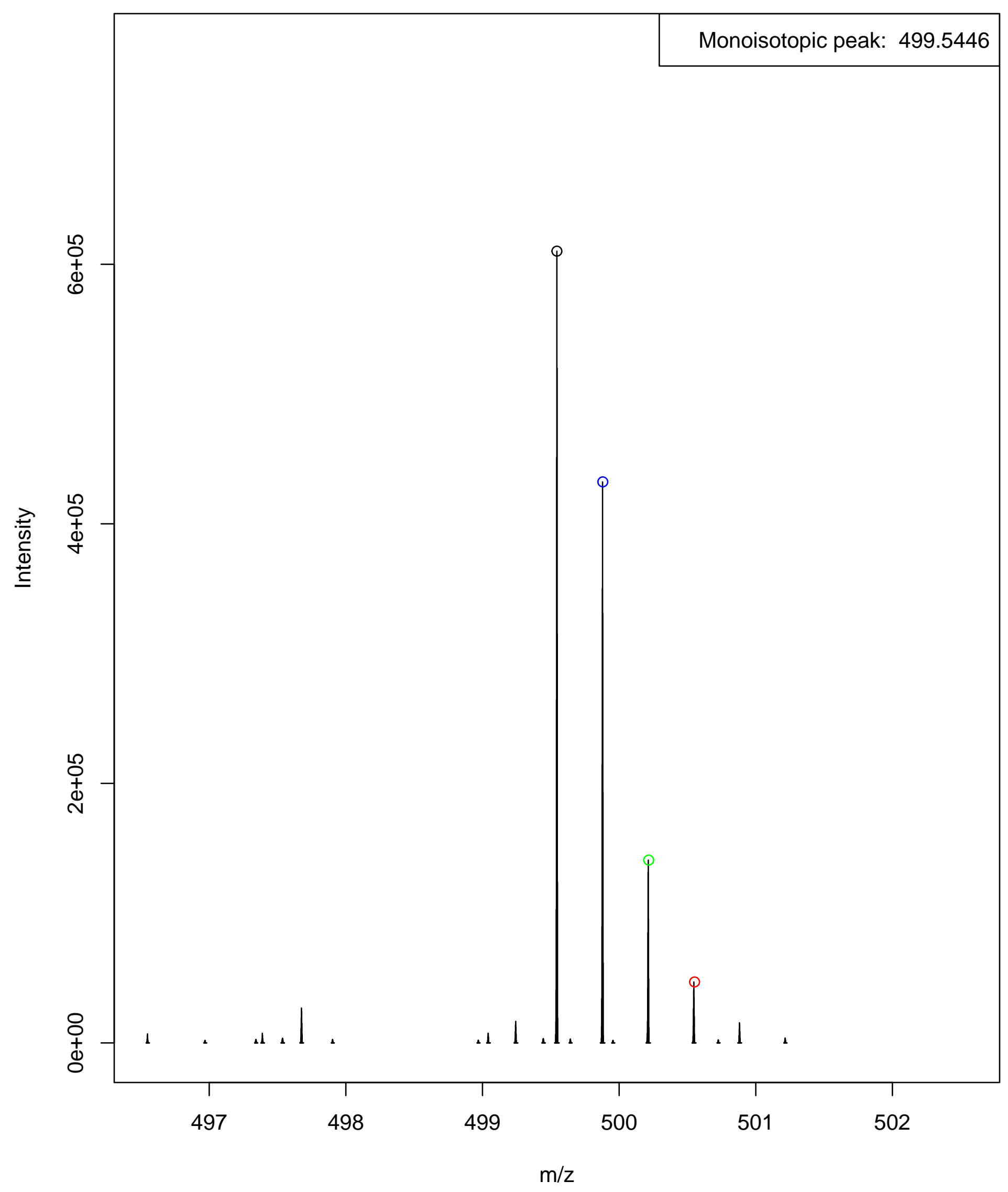
charge $=3$ 

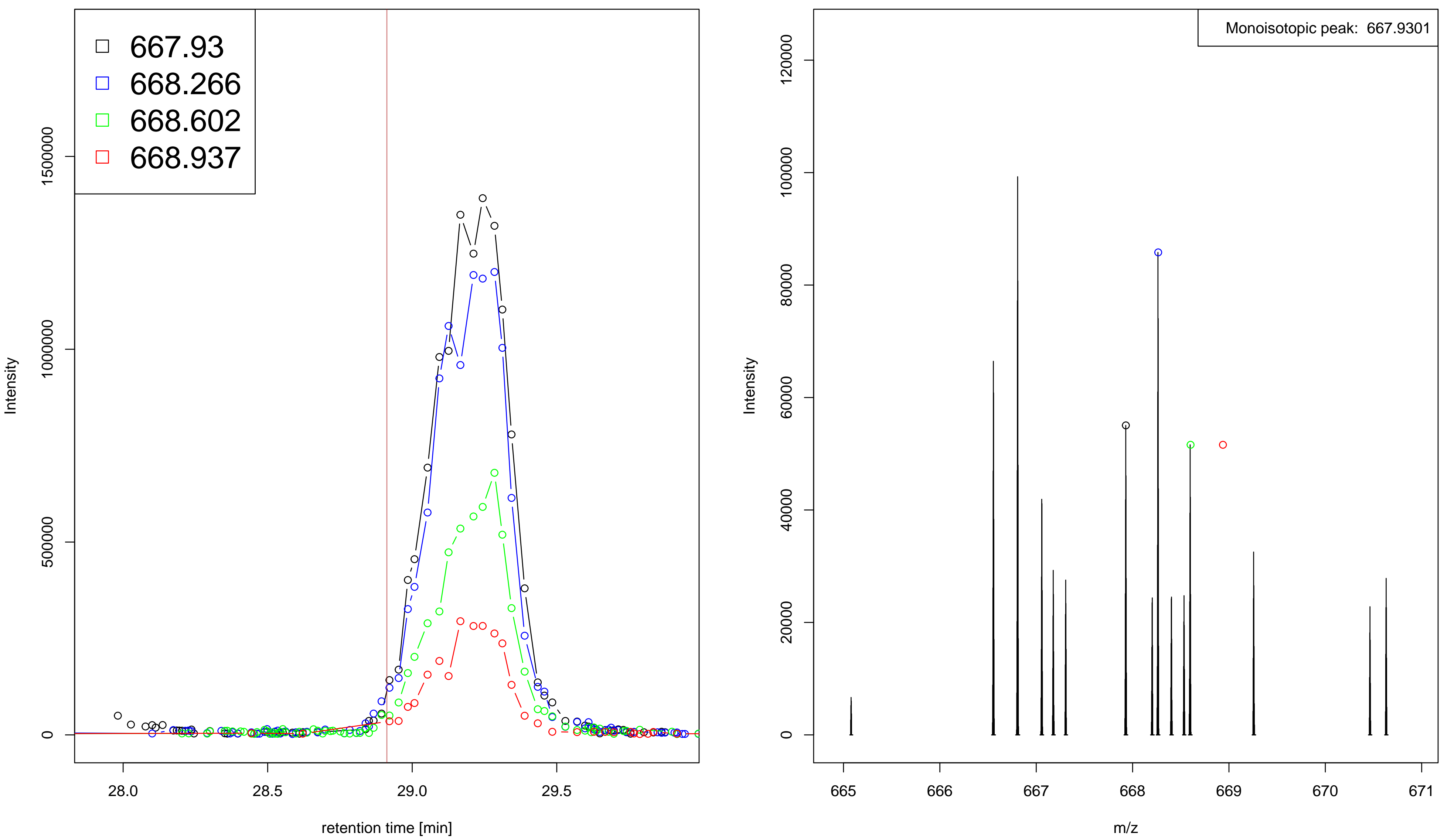

$$
\text { charge }=3
$$


XIC of MS1

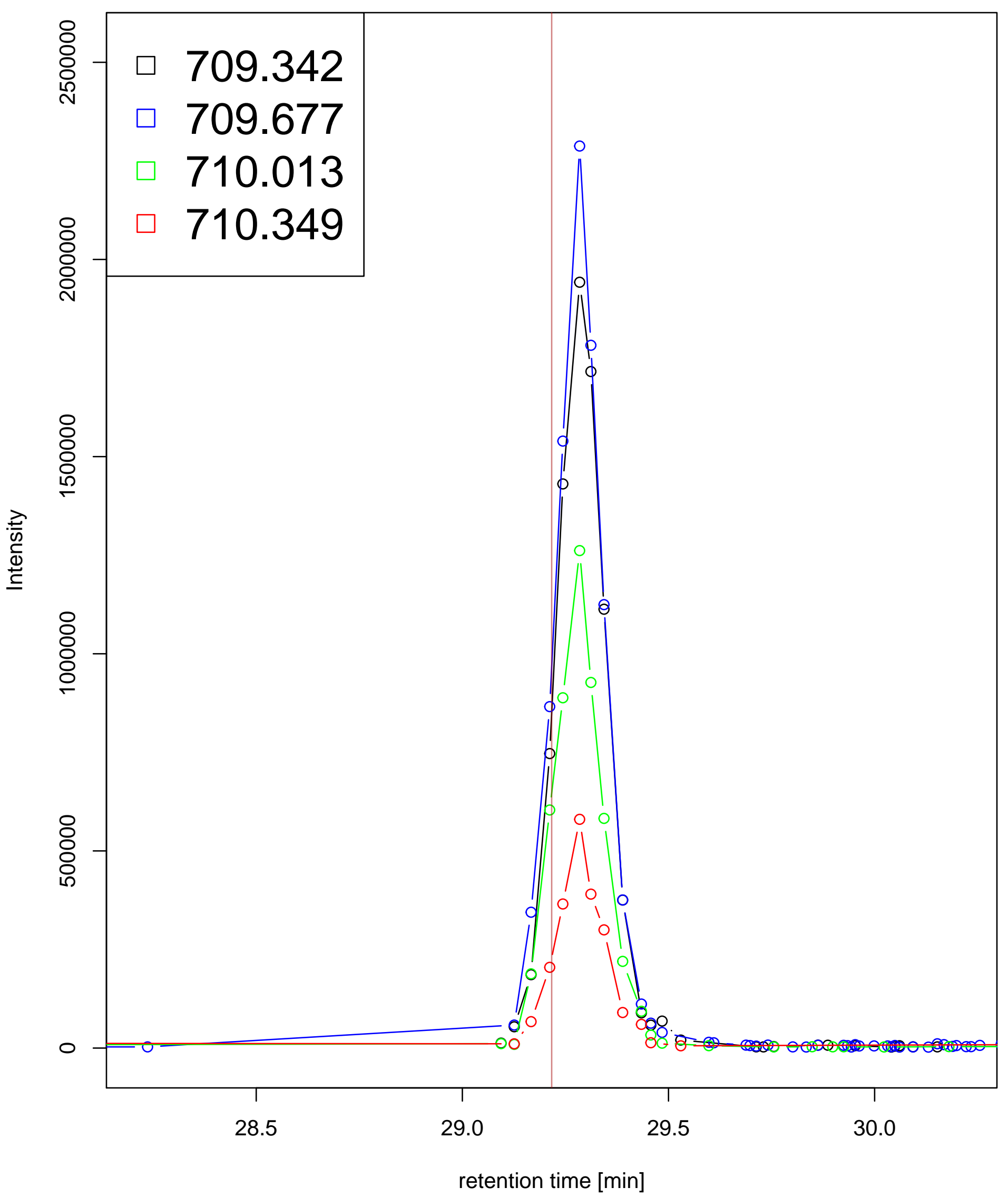

MS1 of ScanNo:4225

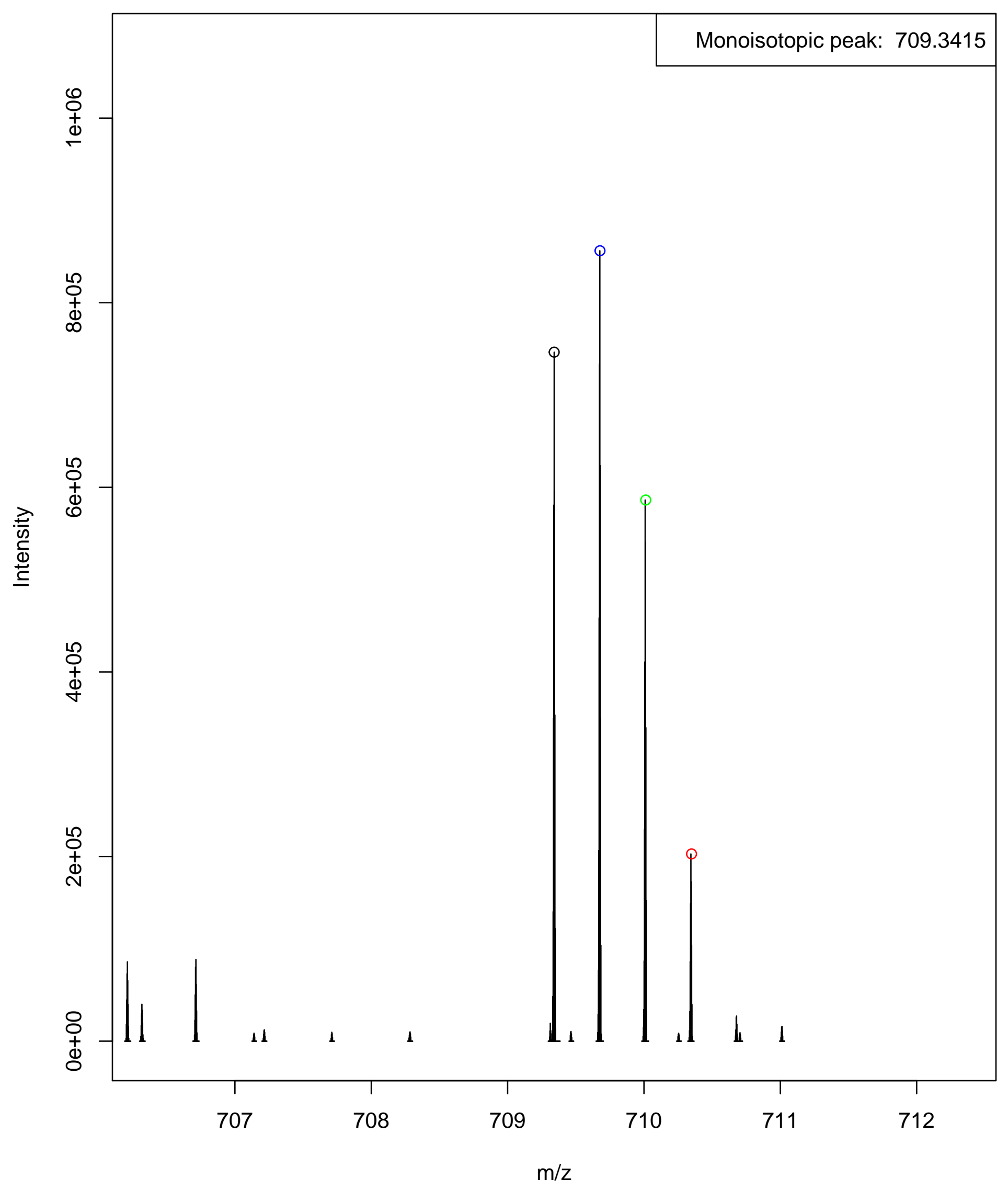


XIC of MS1

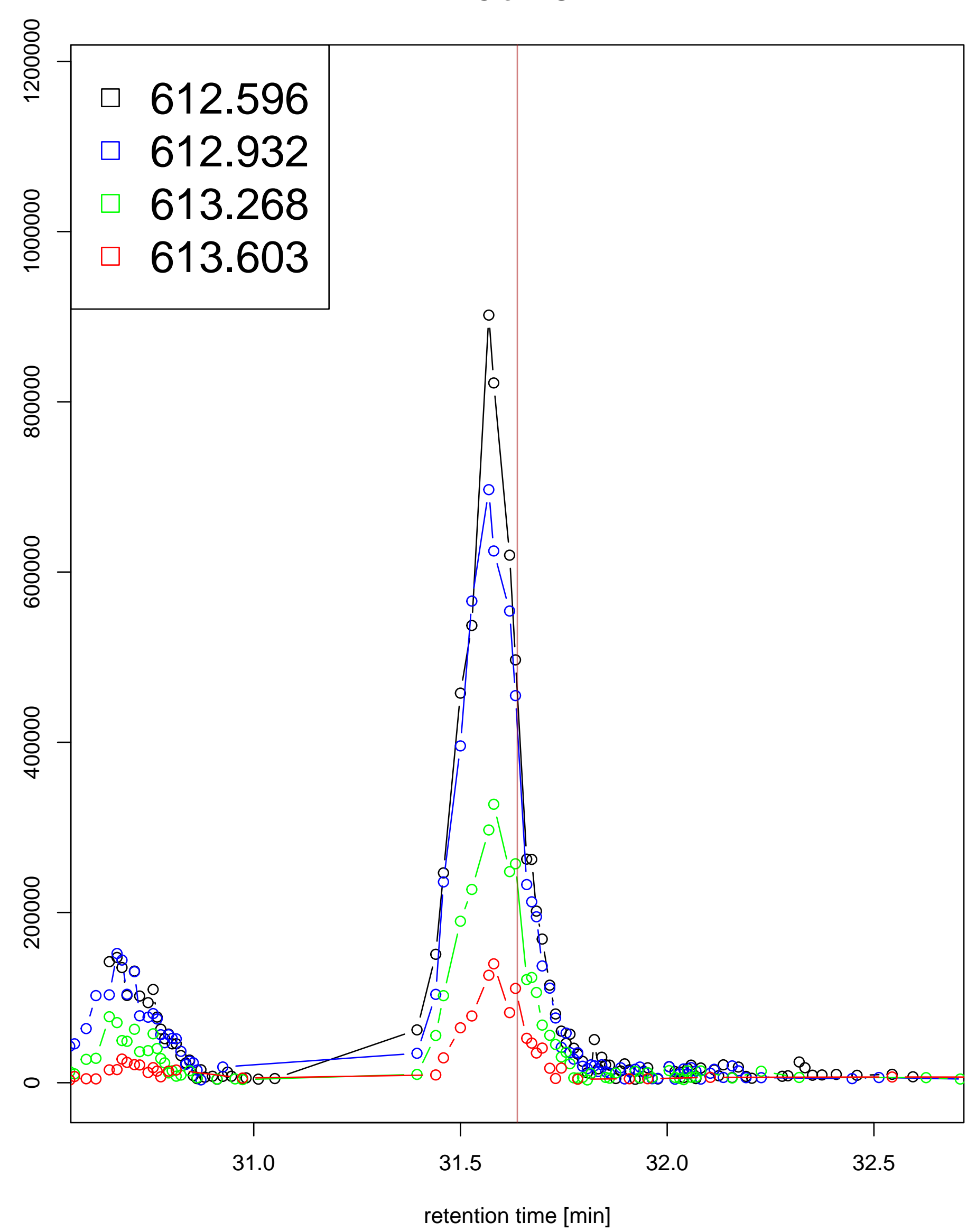

MS1 of ScanNo:4715

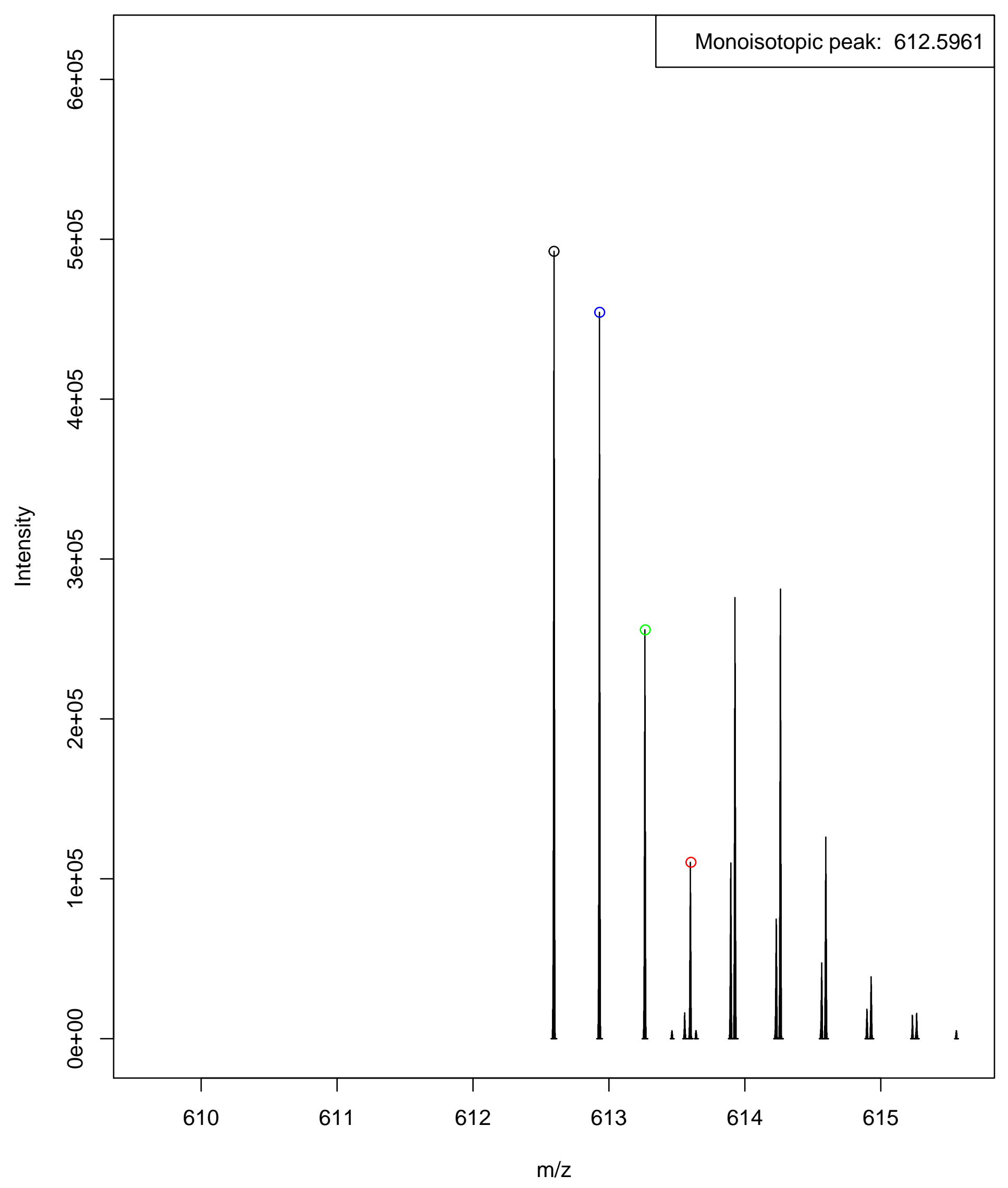
charge $=3$ 

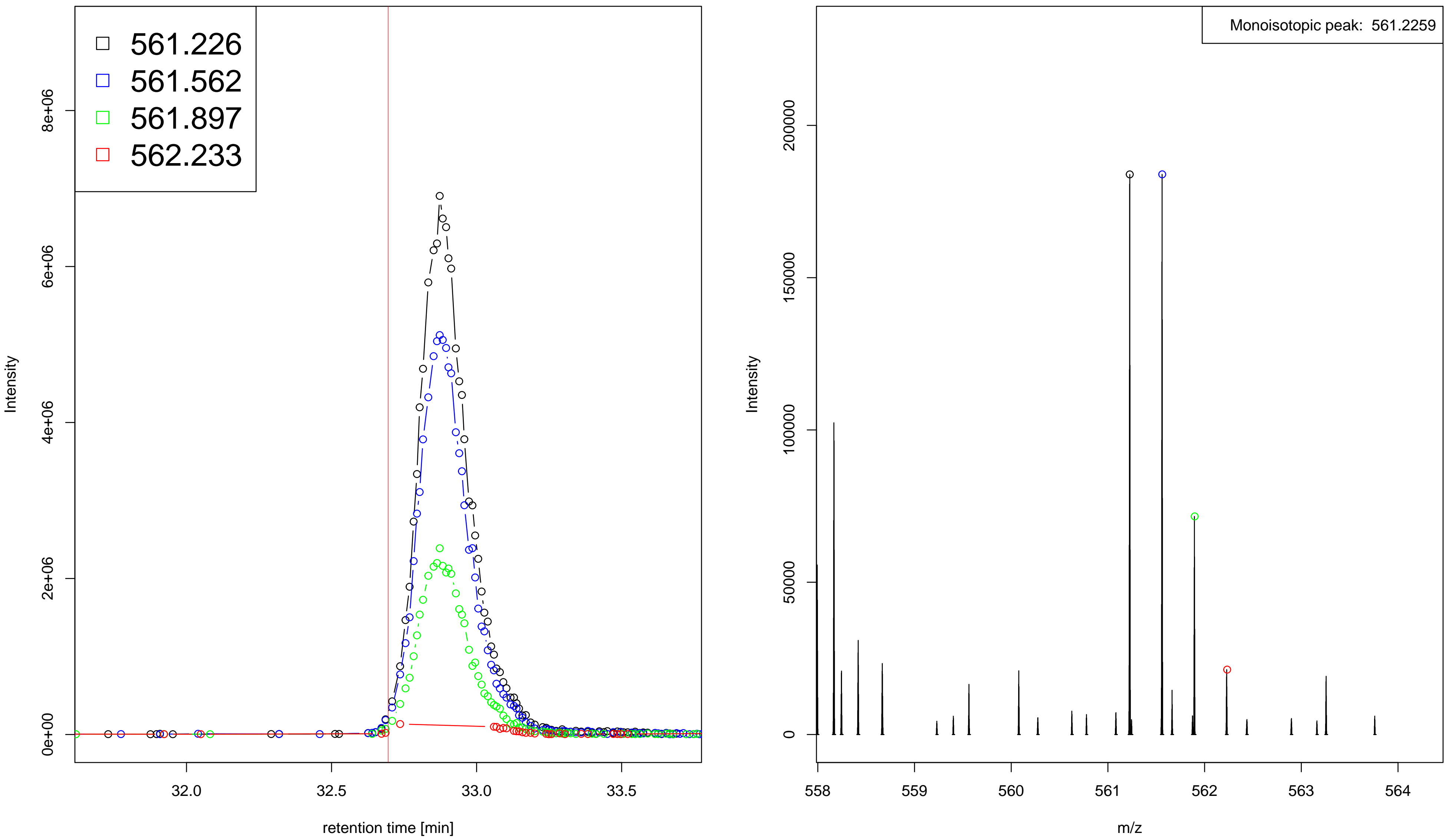

charge $=3$ 
XIC of MS1

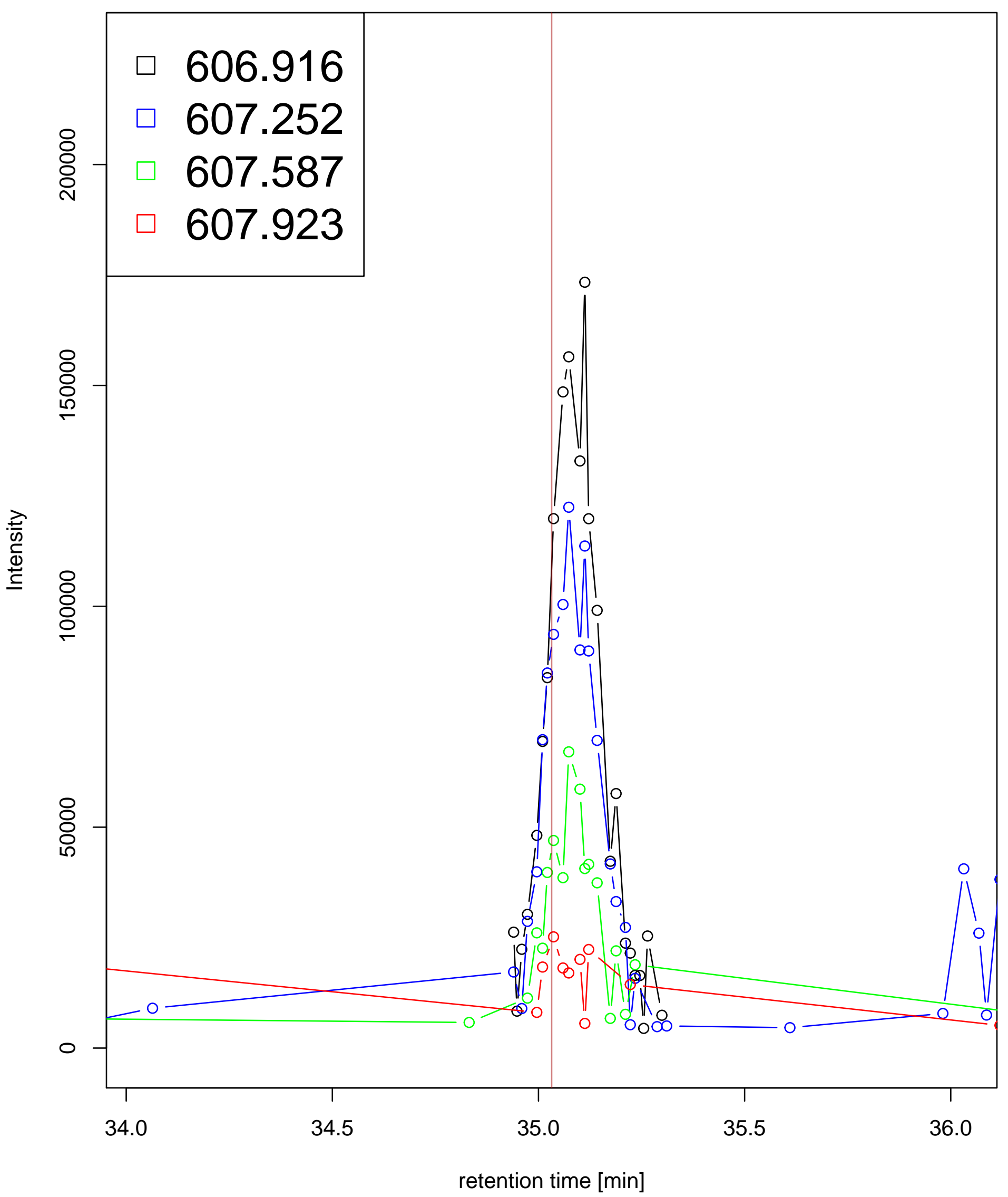

MS1 of ScanNo:5421

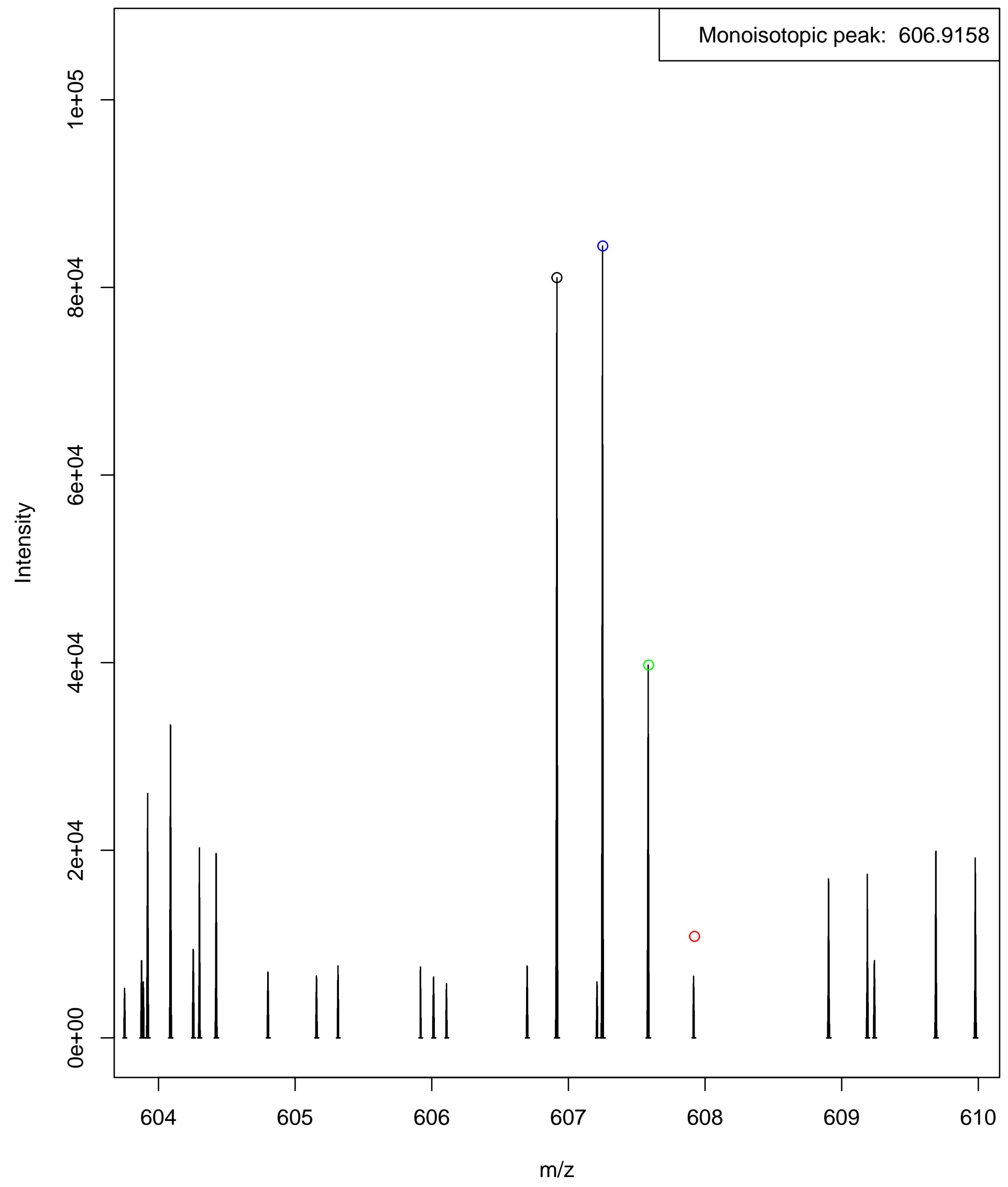


XIC of MS1

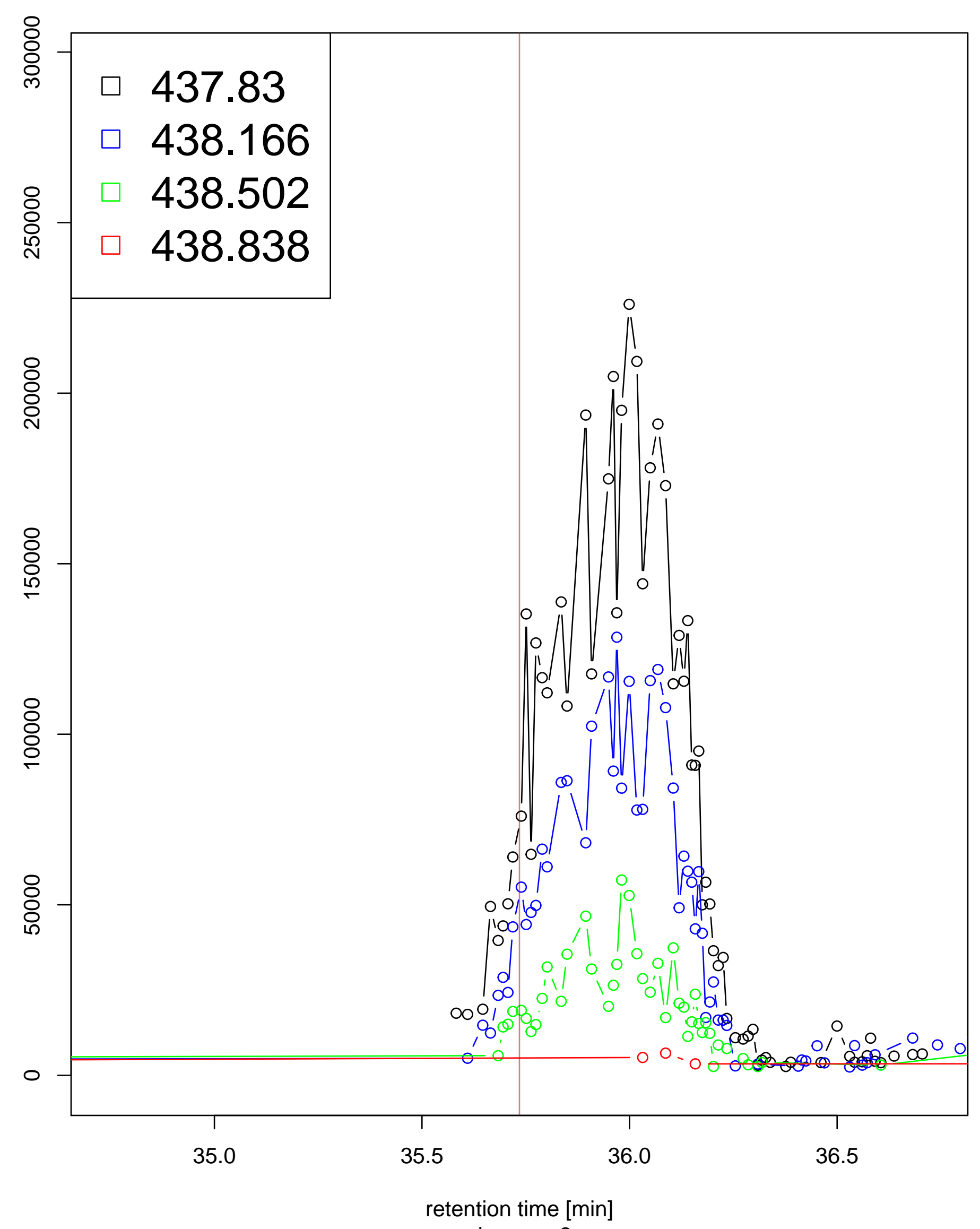

MS1 of ScanNo:5571

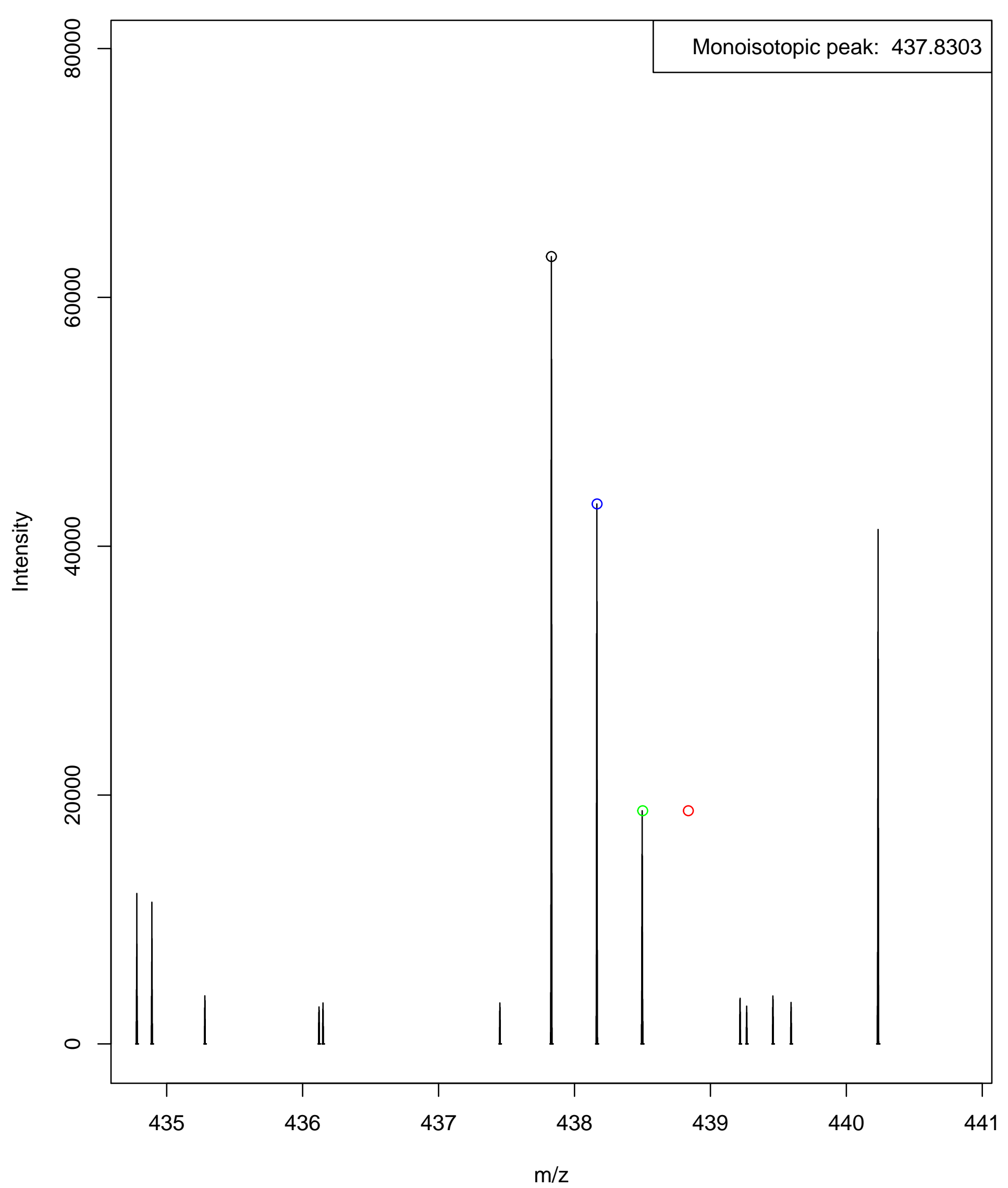



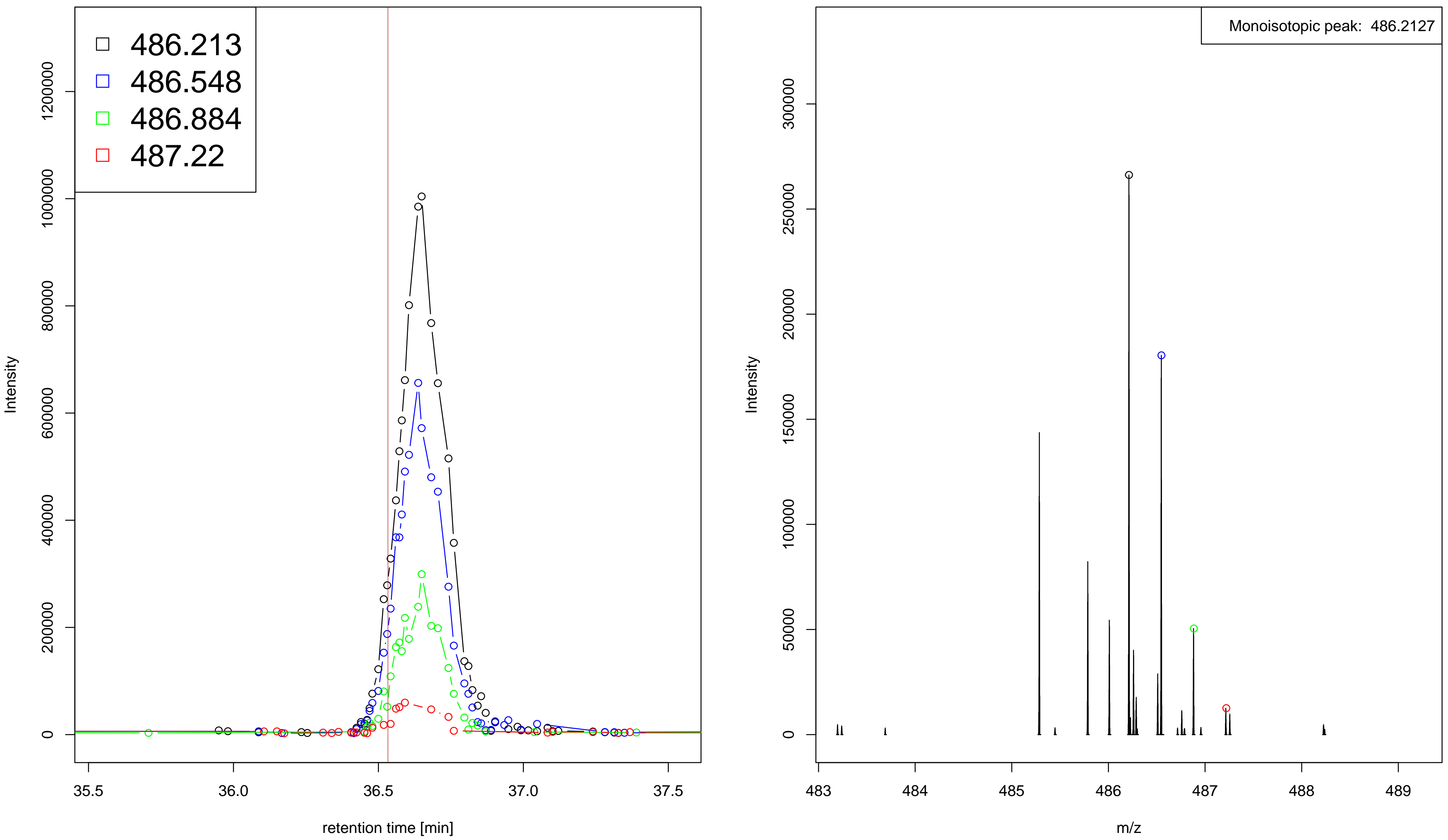
XIC of MS1

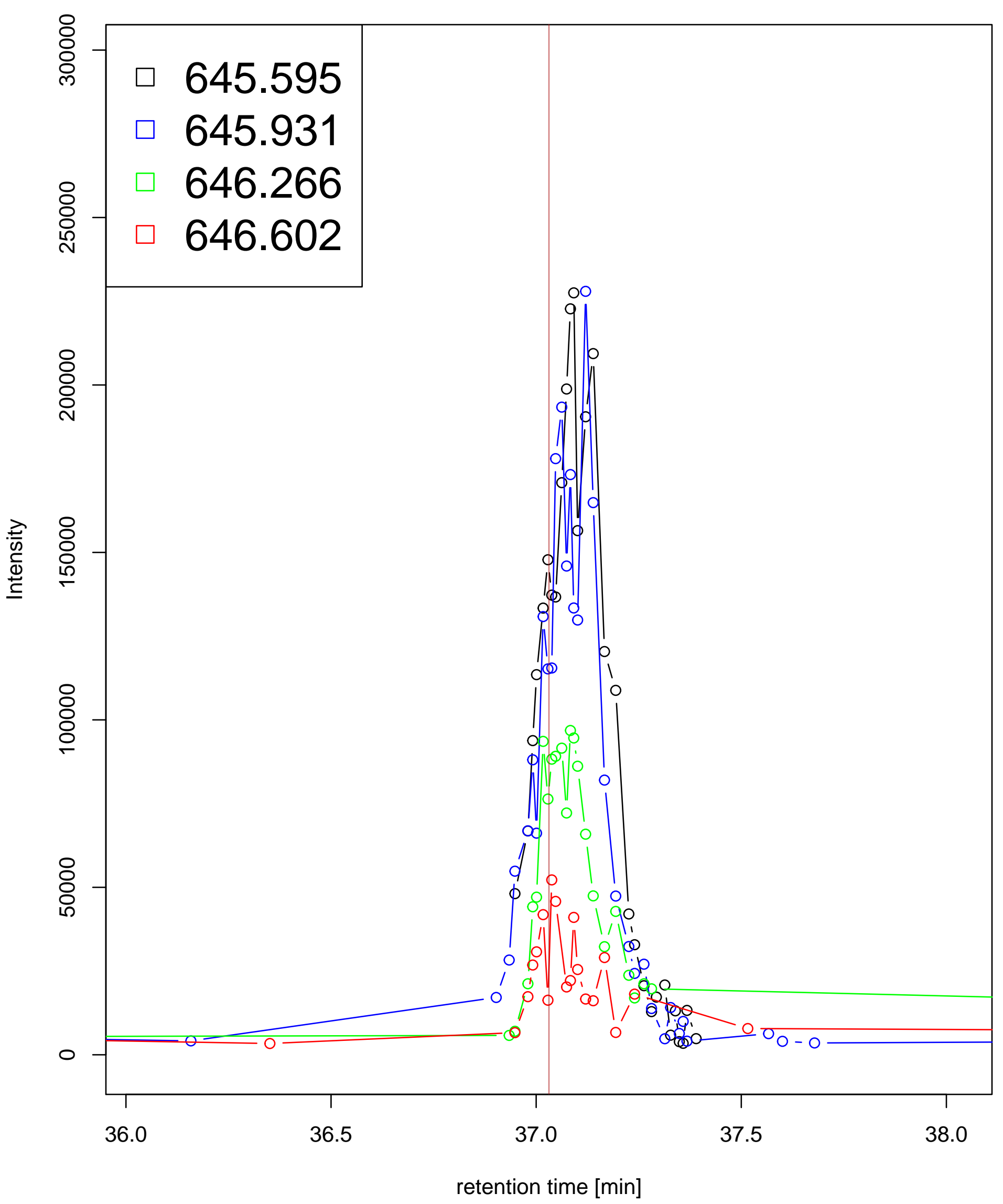

MS1 of ScanNo:5839

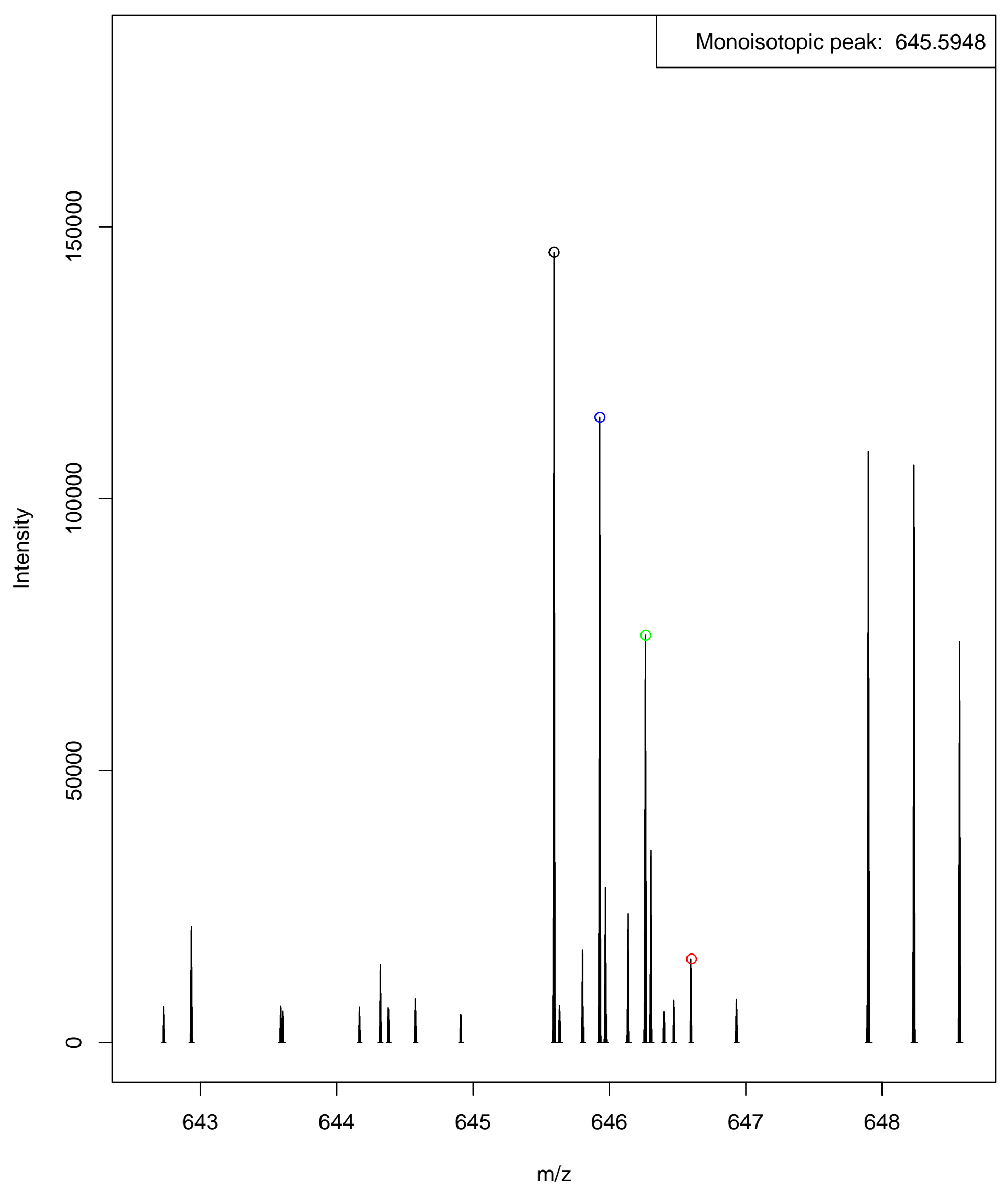


XIC of MS1

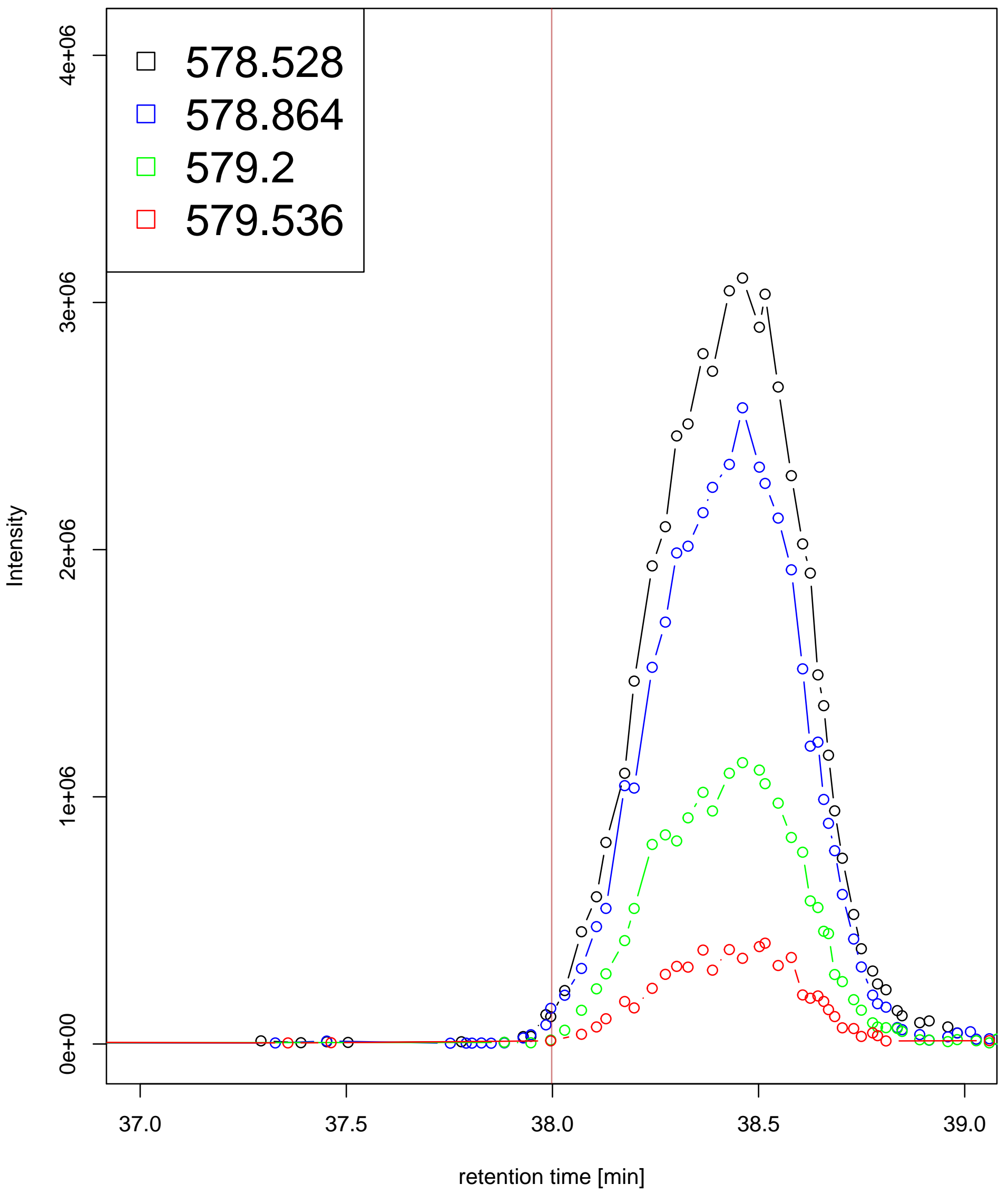

MS1 of ScanNo:6044

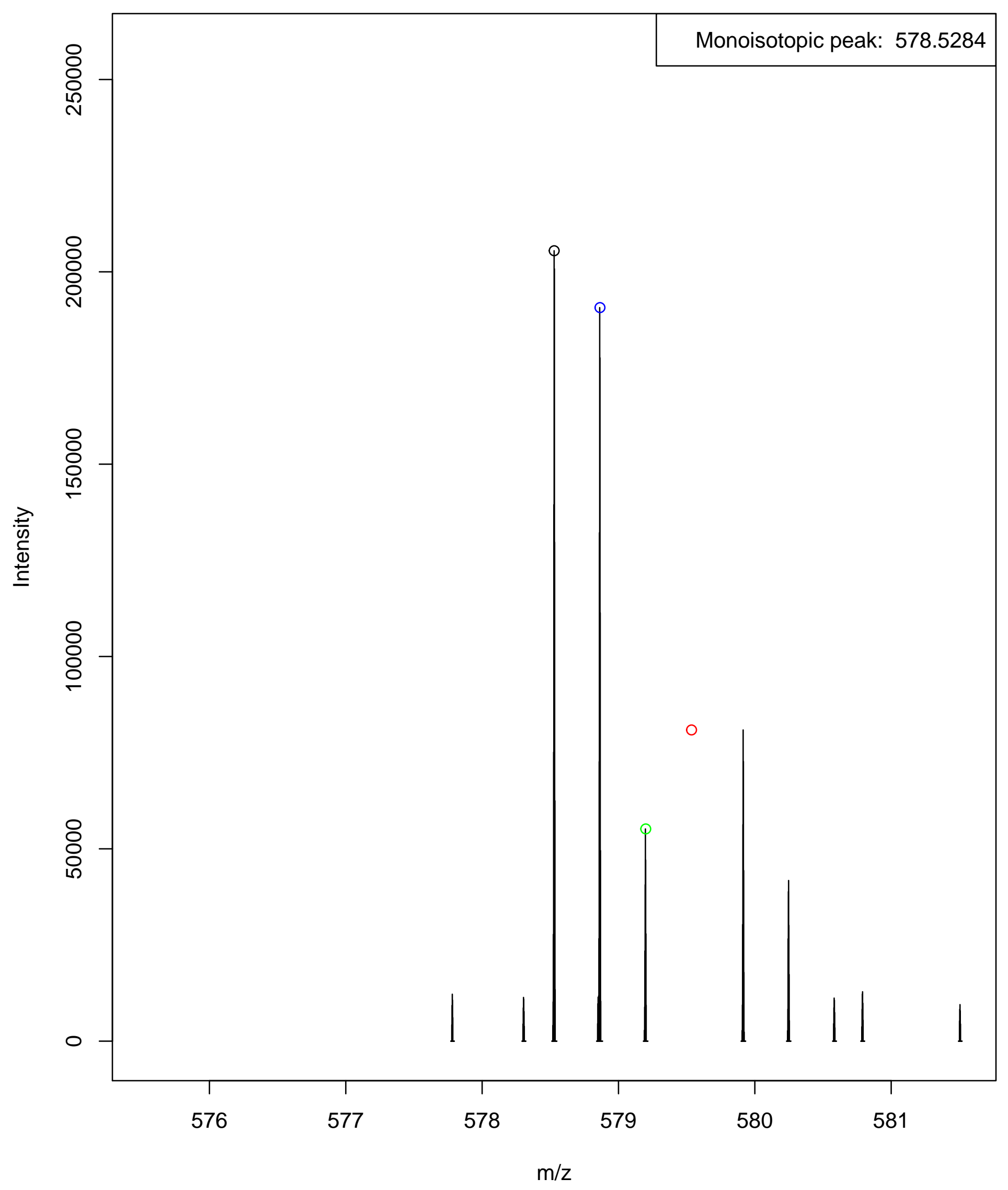


XIC of MS1

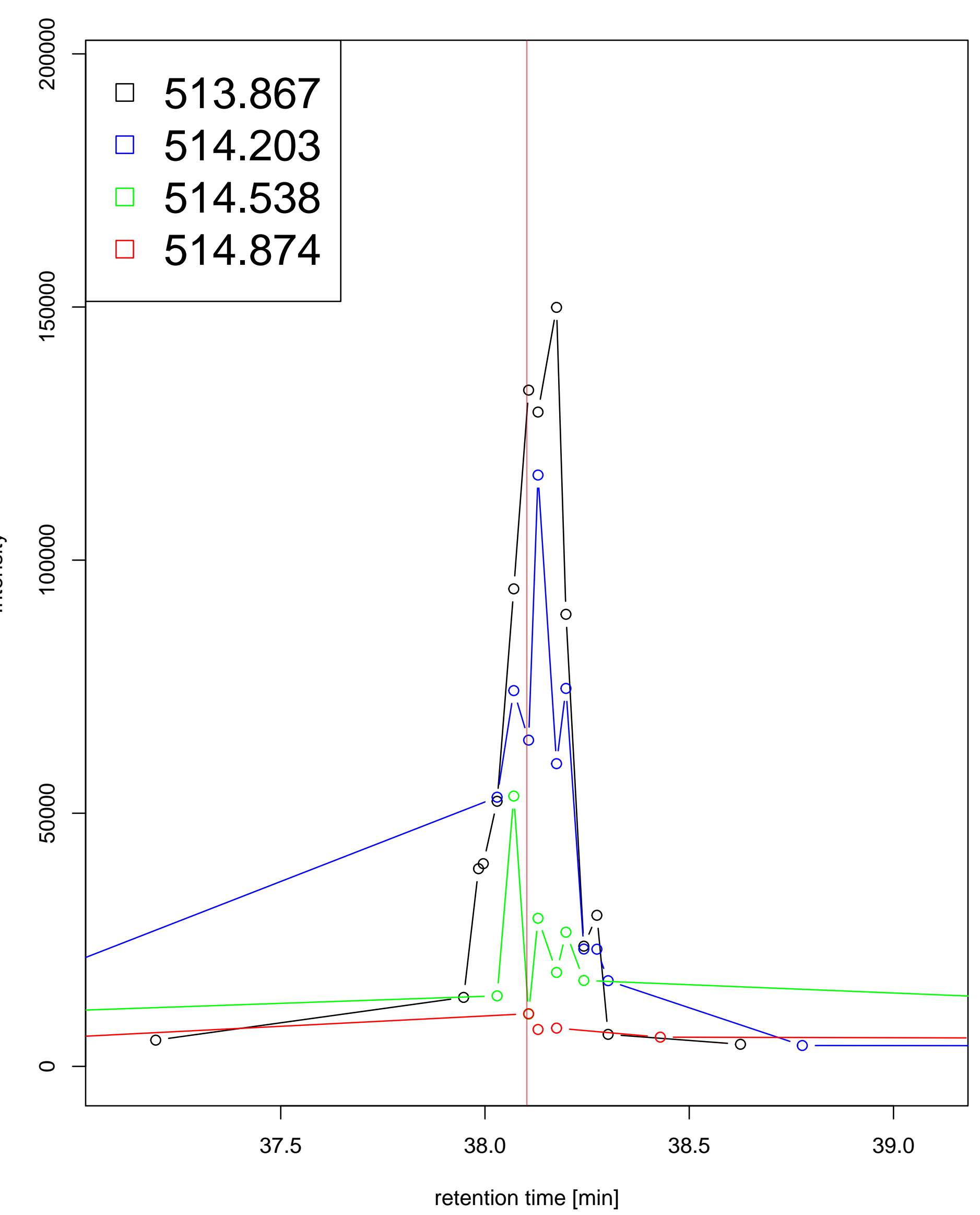

MS1 of ScanNo:6053

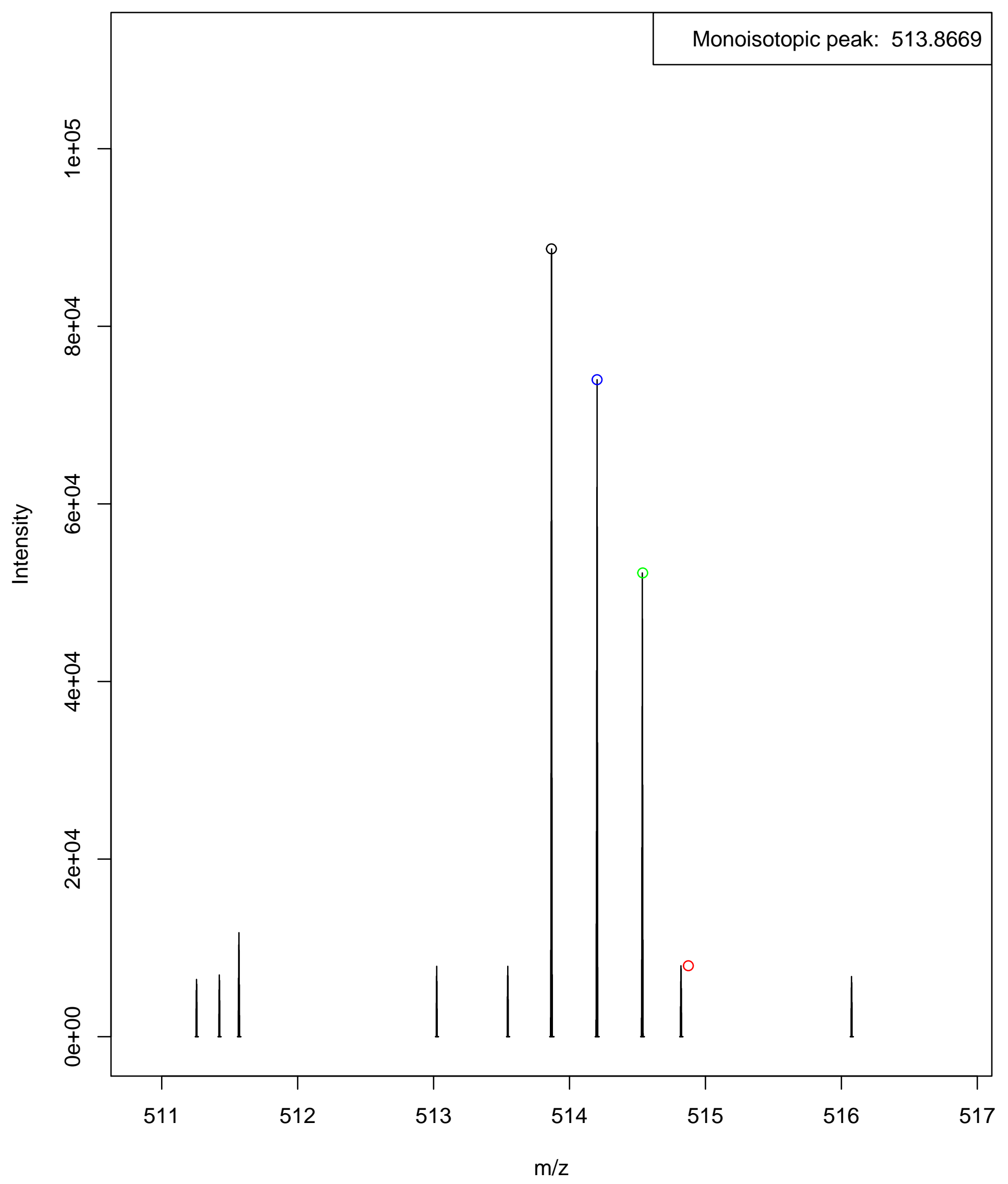
charge $=3$ 

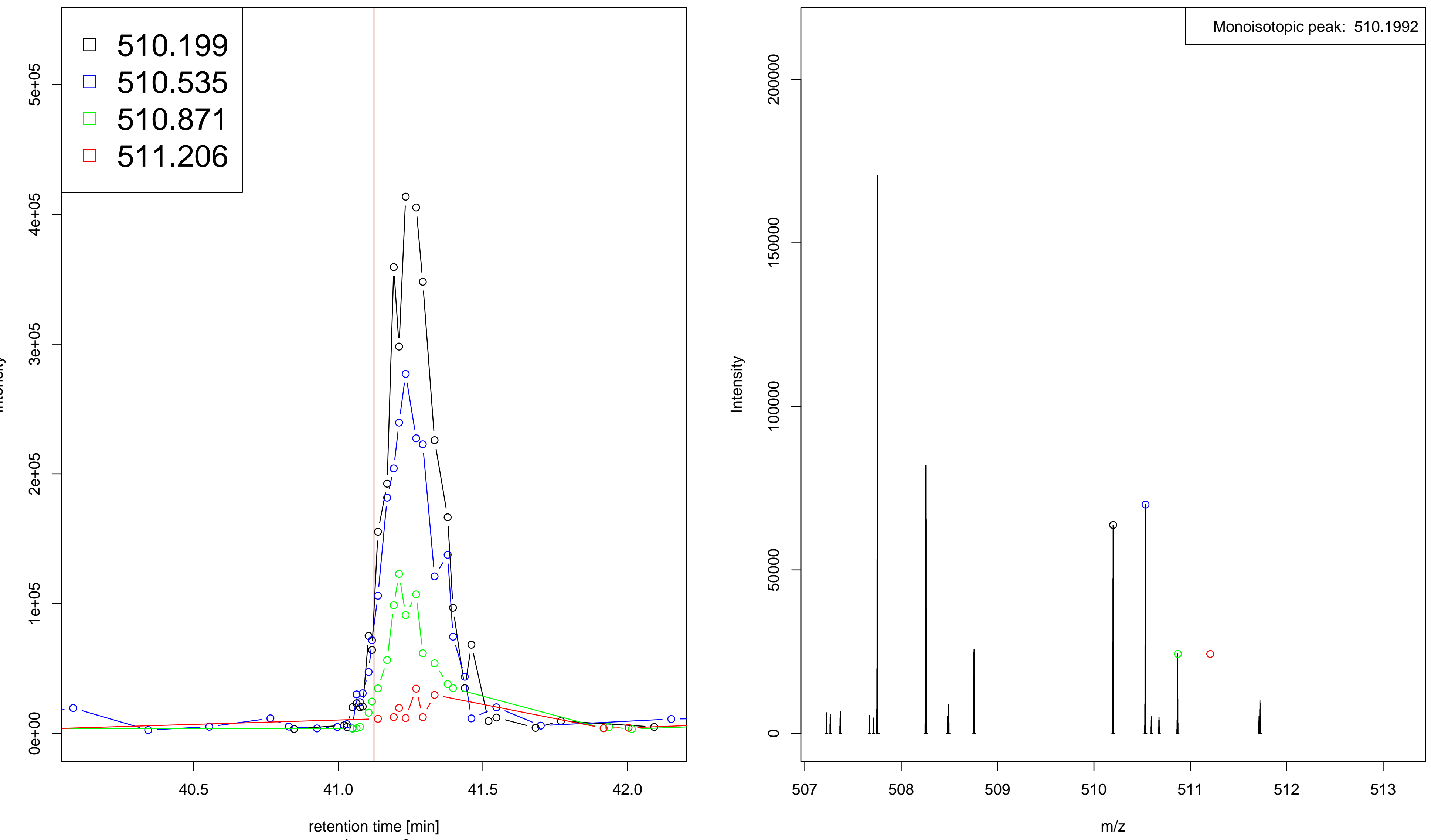

charge $=3$ 
XIC of MS1

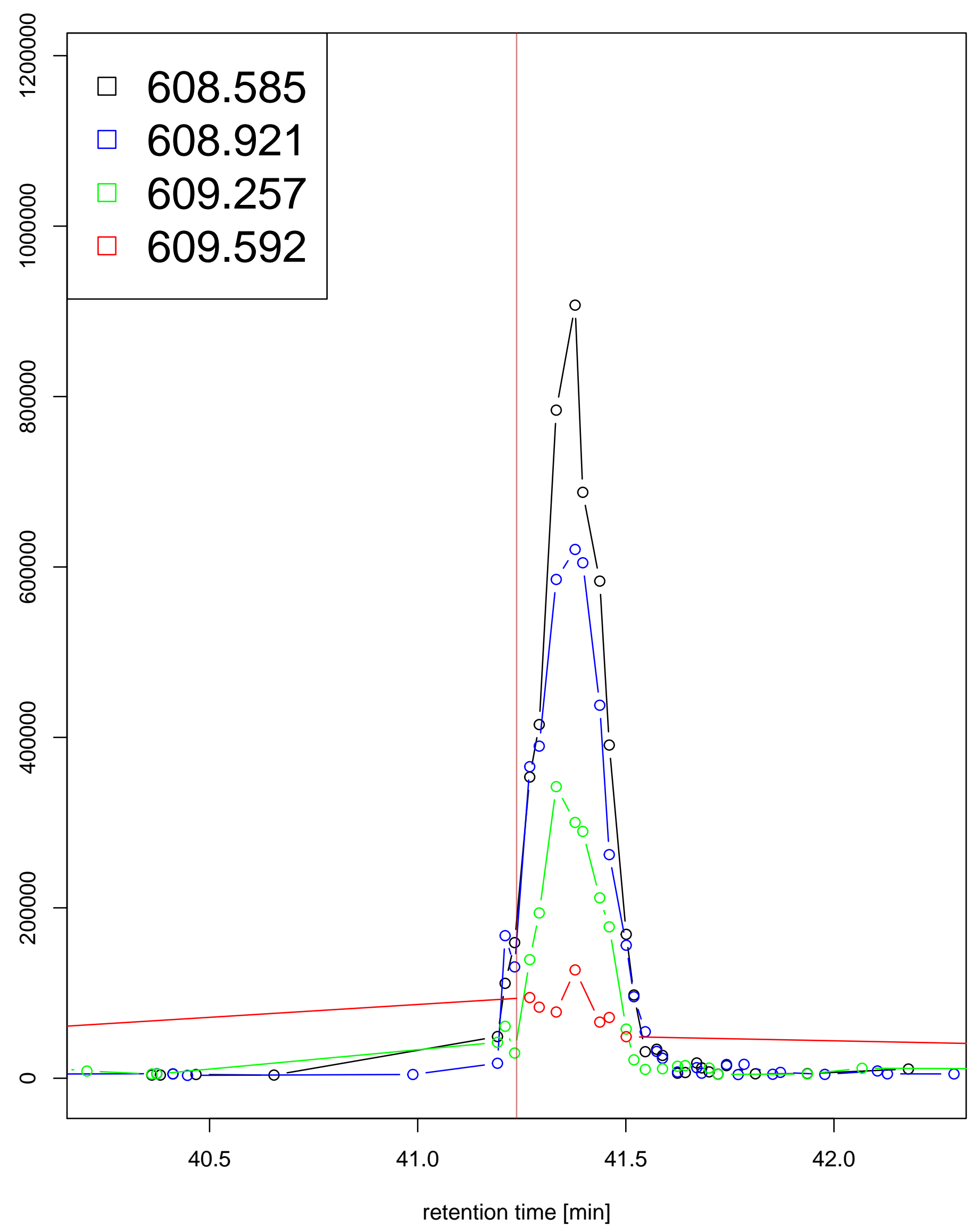

MS1 of ScanNo:6723

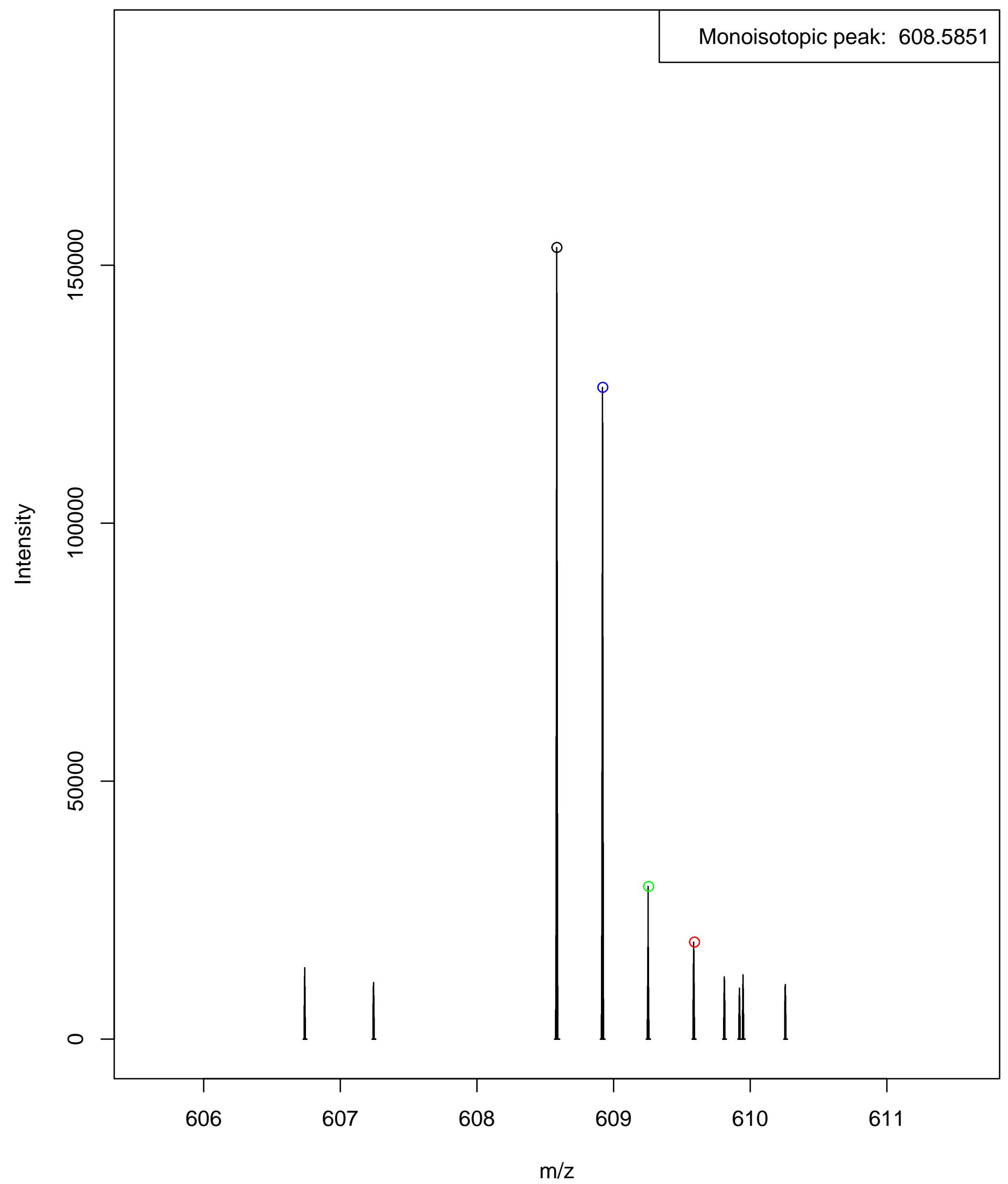


XIC of MS1

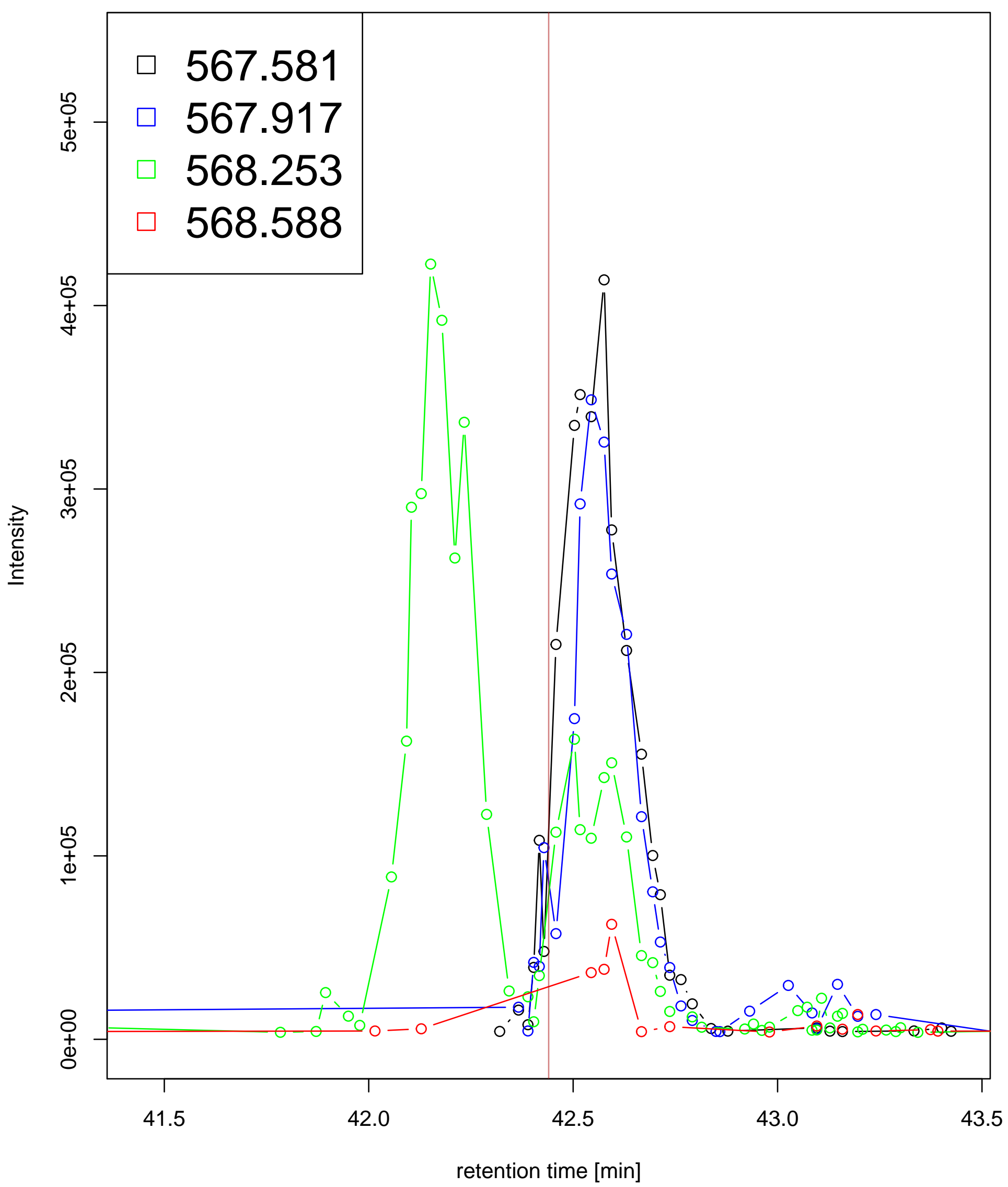

MS1 of ScanNo:6981

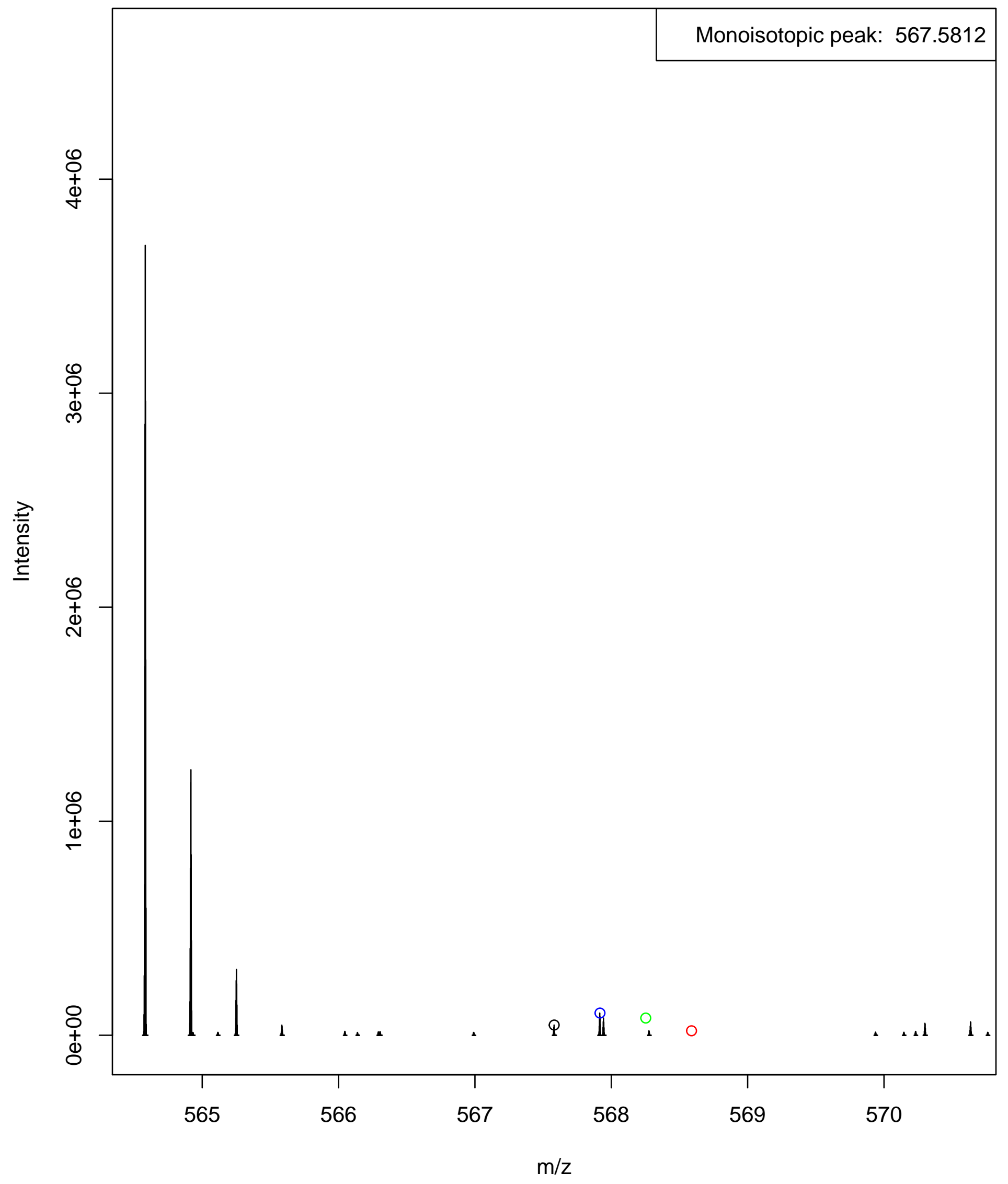


XIC of MS1

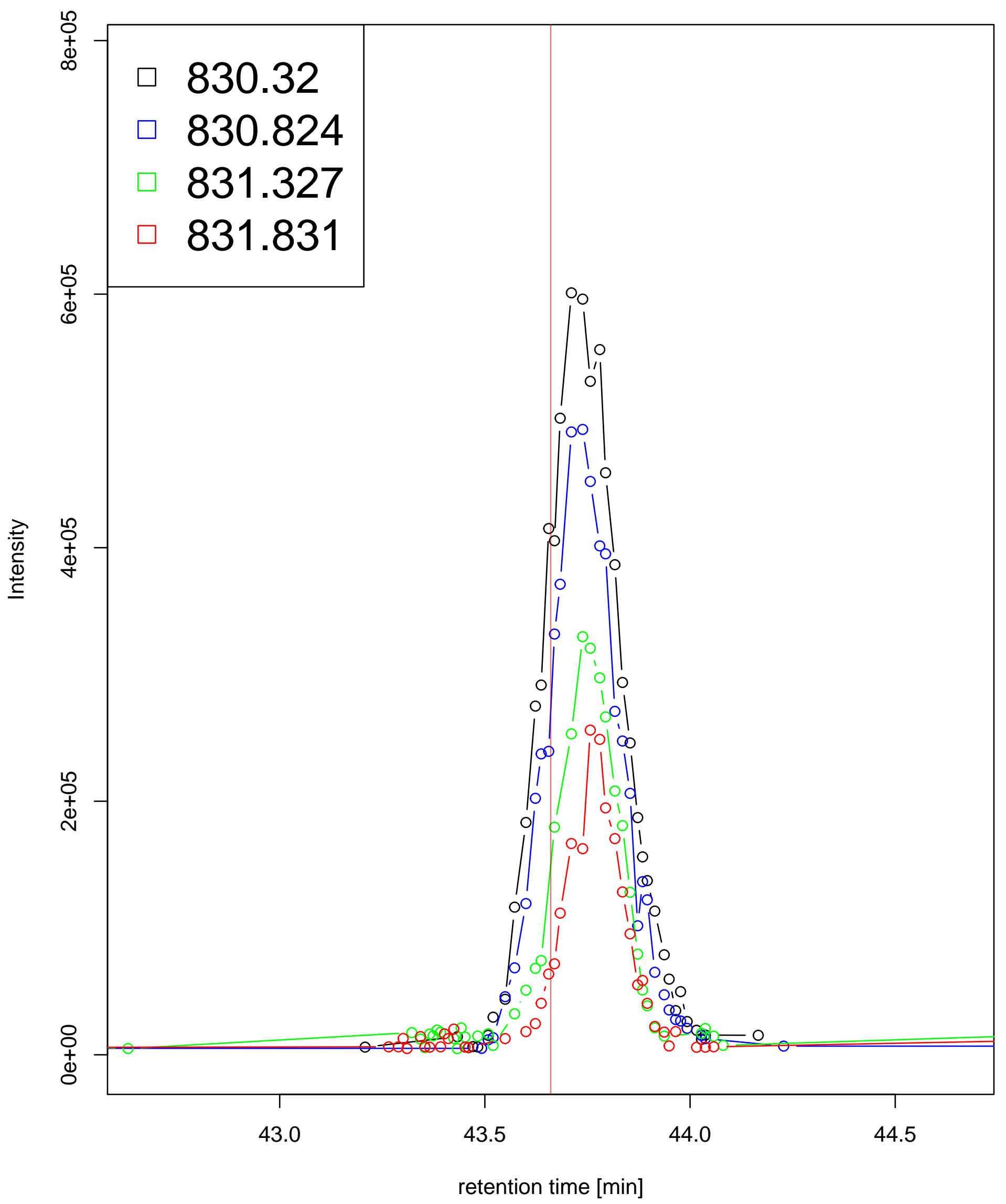

MS1 of ScanNo:7238

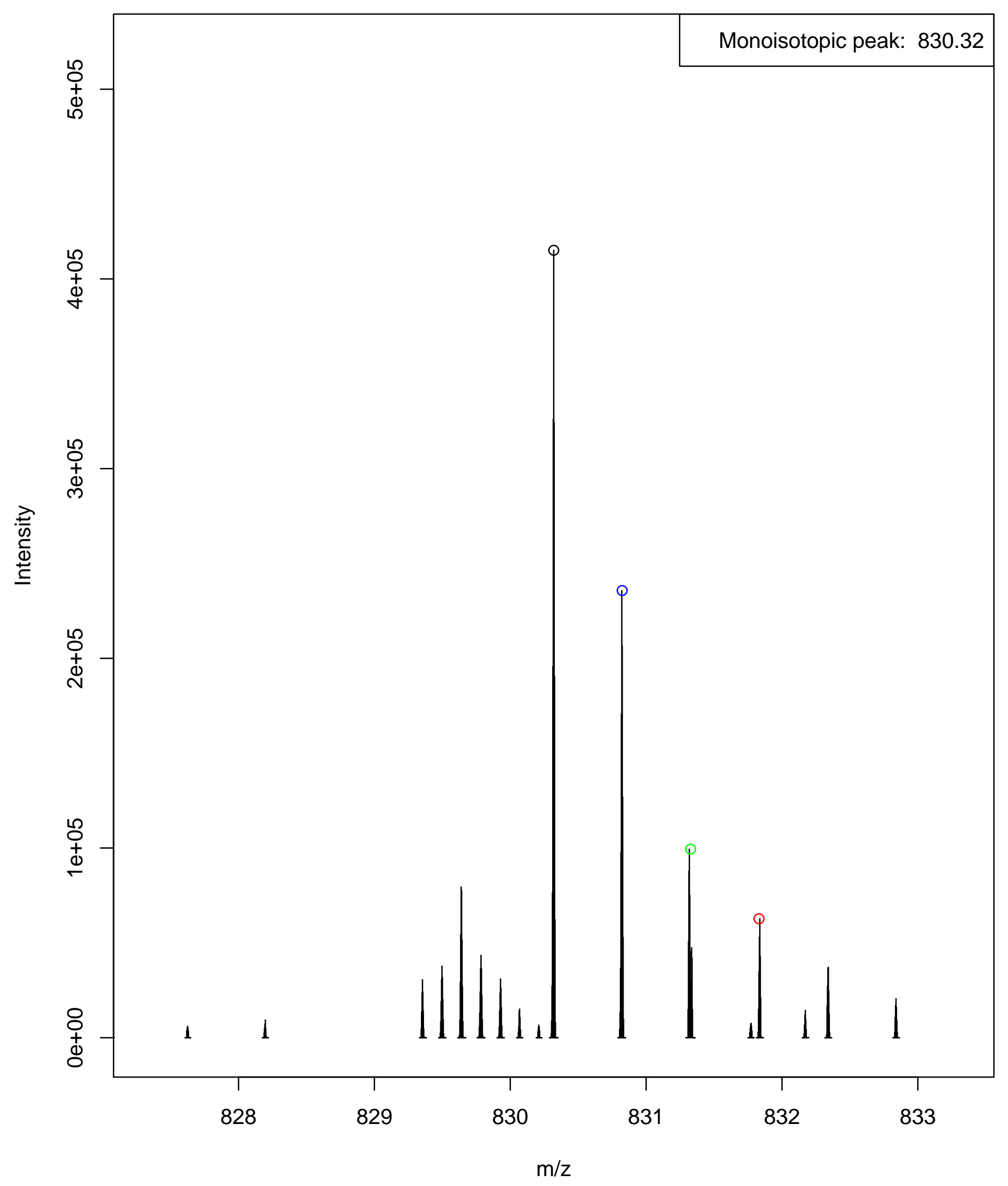
charge $=2$ 
XIC of MS1

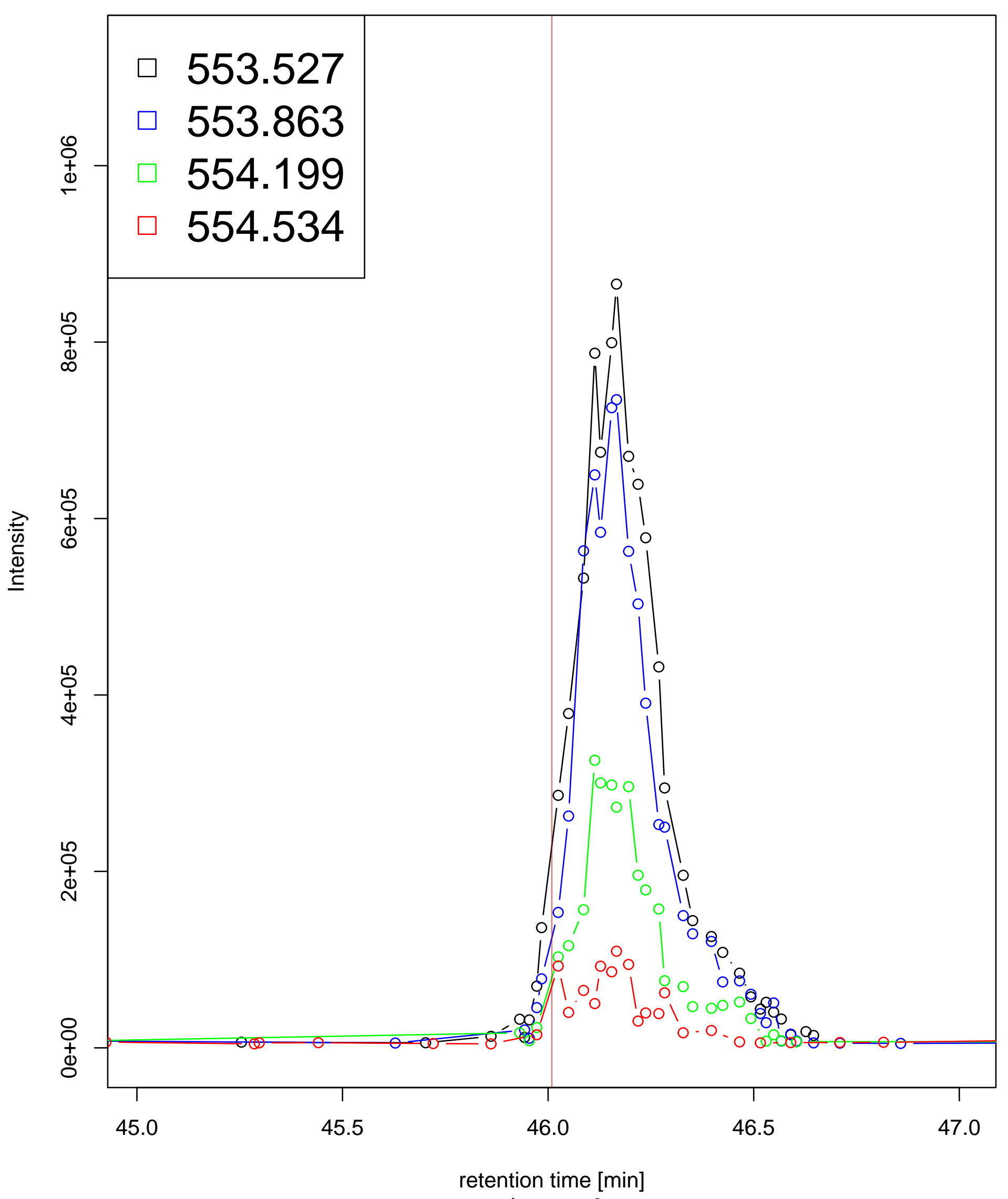

MS1 of ScanNo:7745

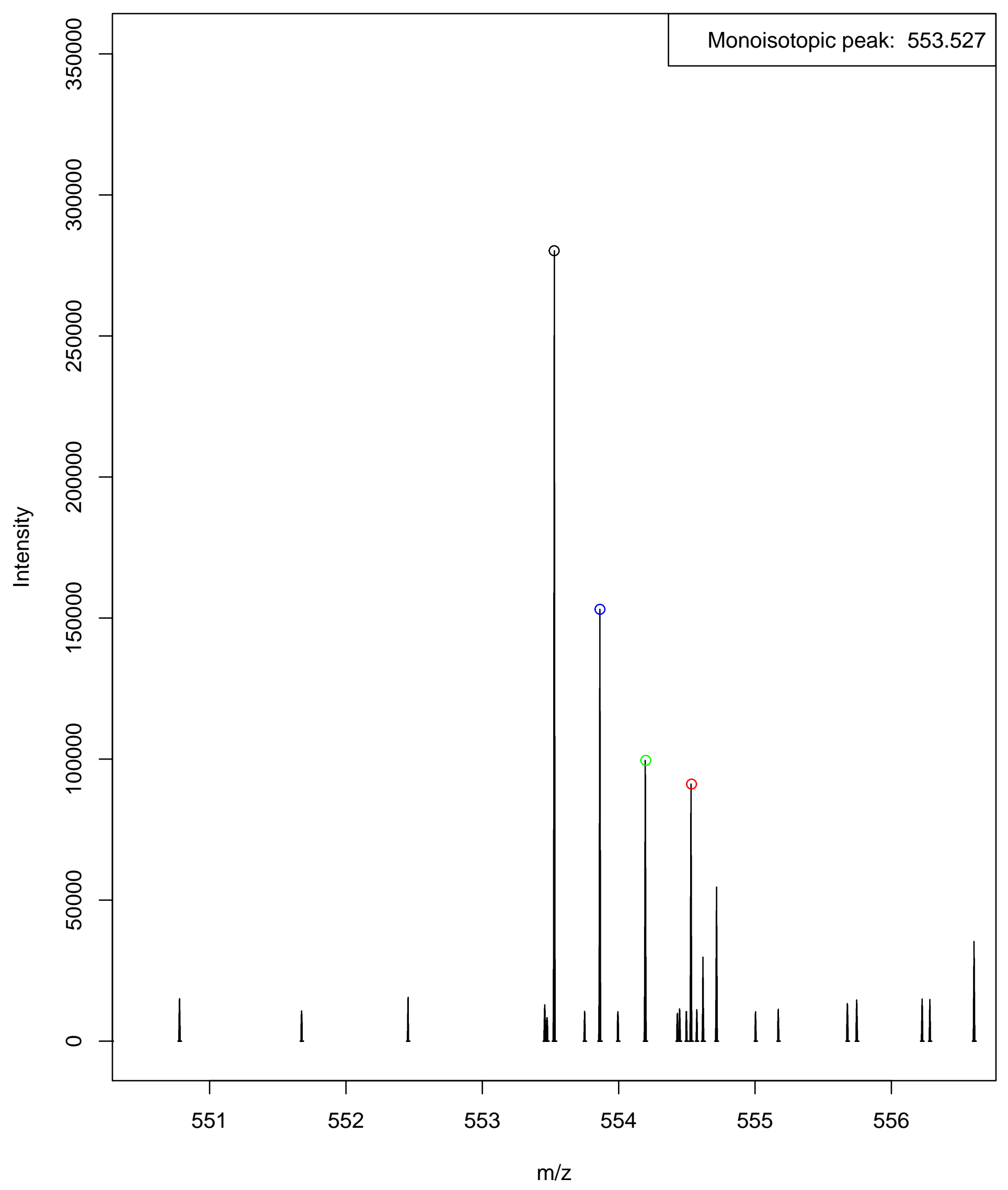


XIC of MS1

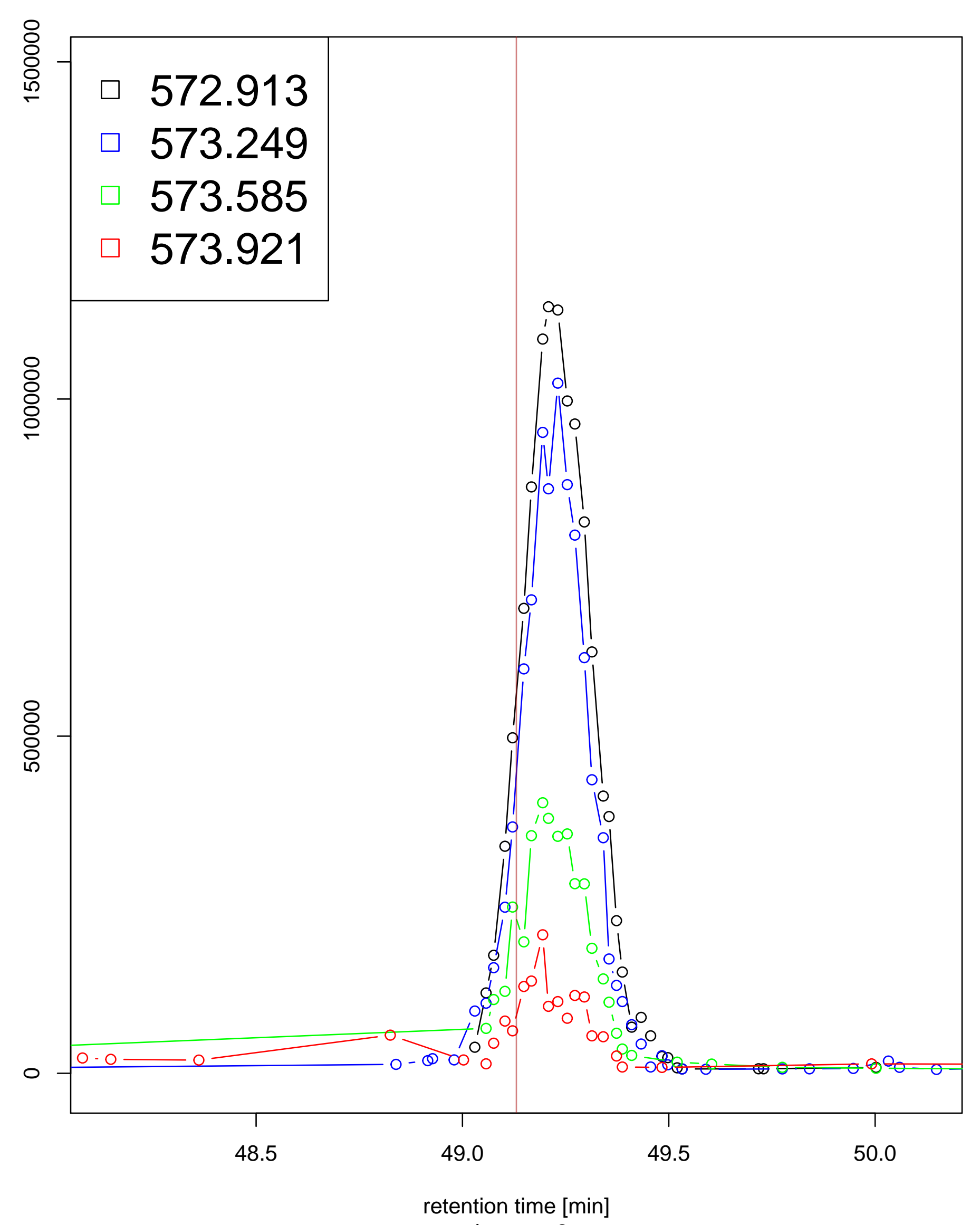

MS1 of ScanNo:8421

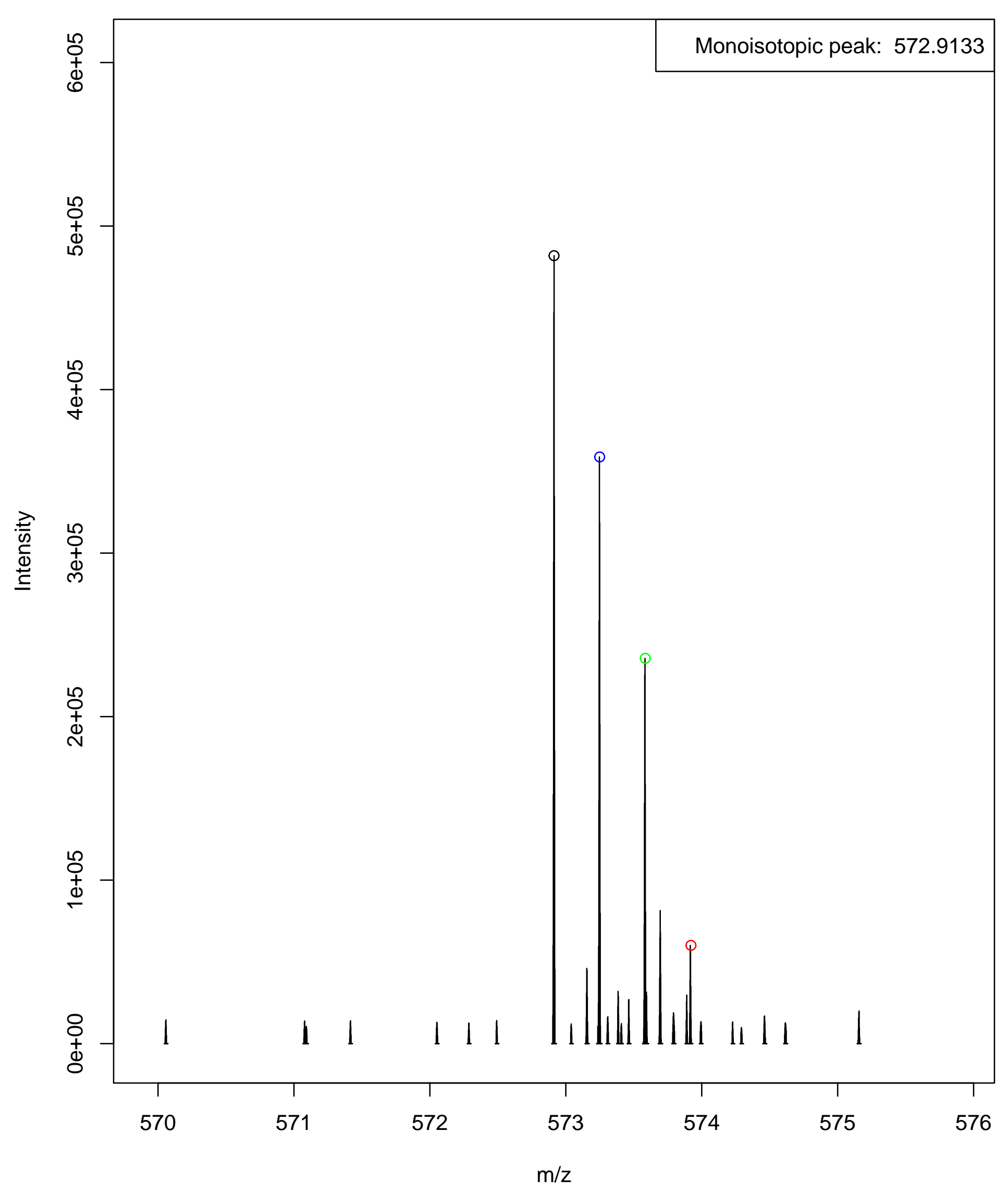
charge $=3$ 
XIC of MS1

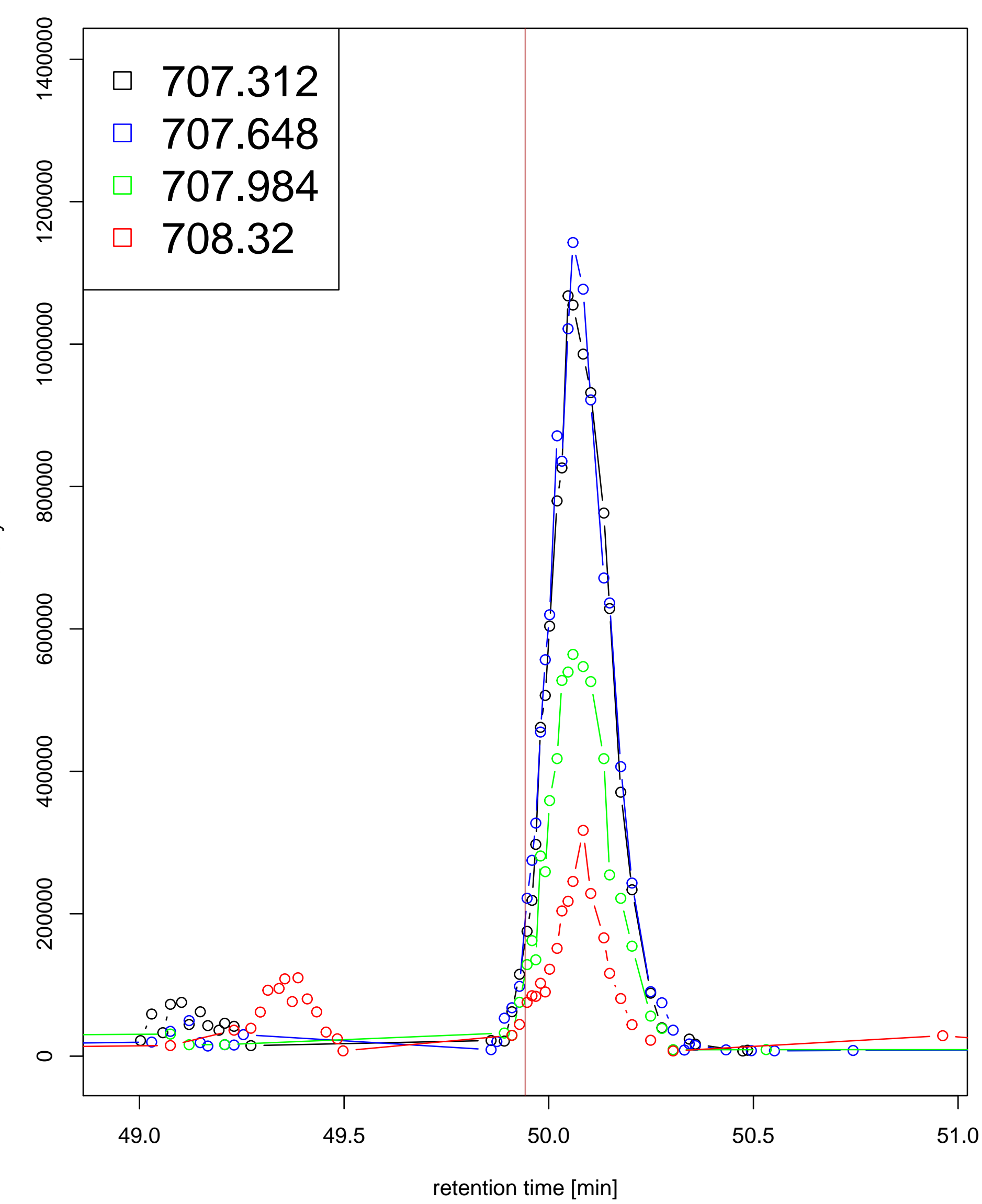

MS1 of ScanNo:8599

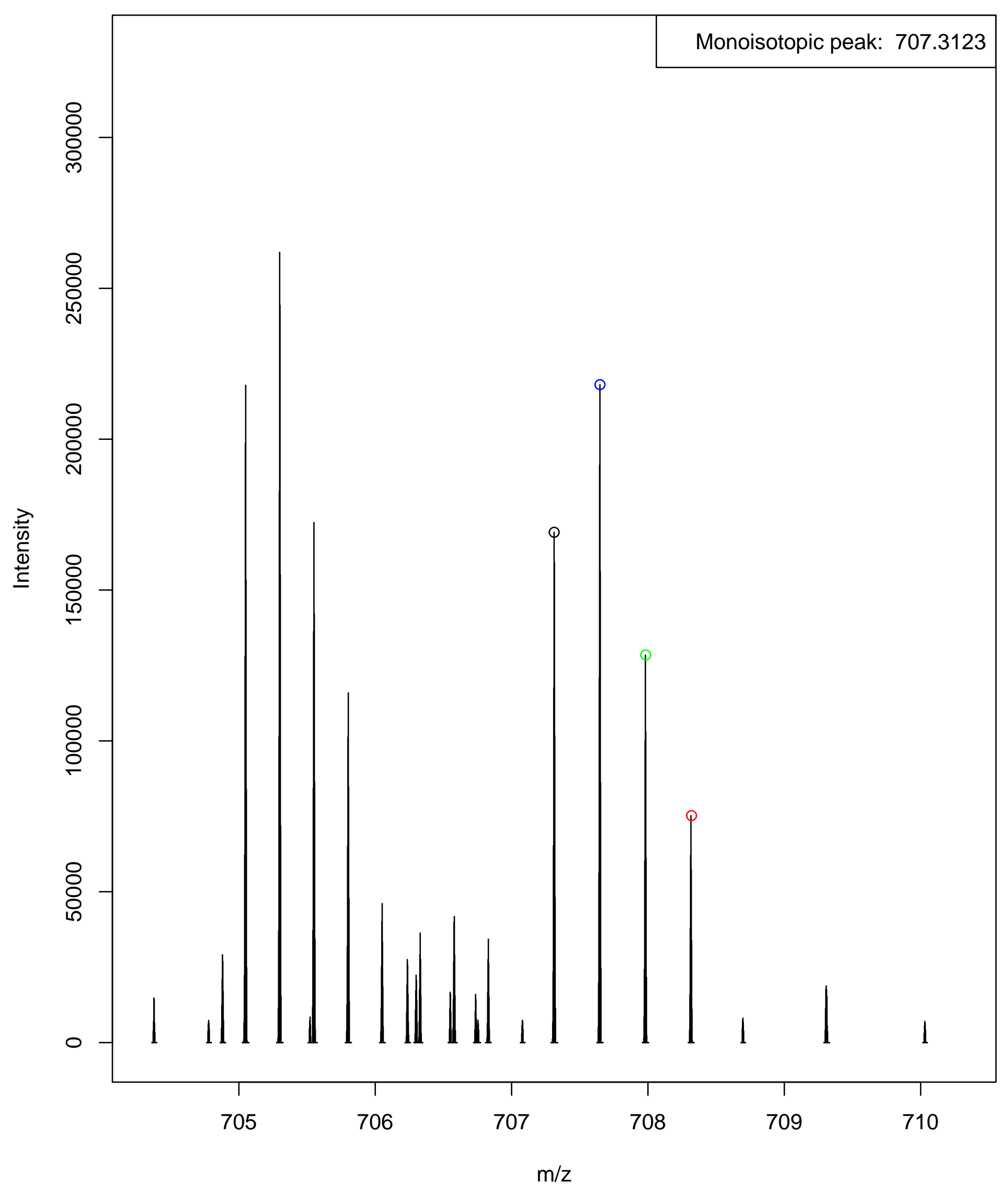


XIC of MS1

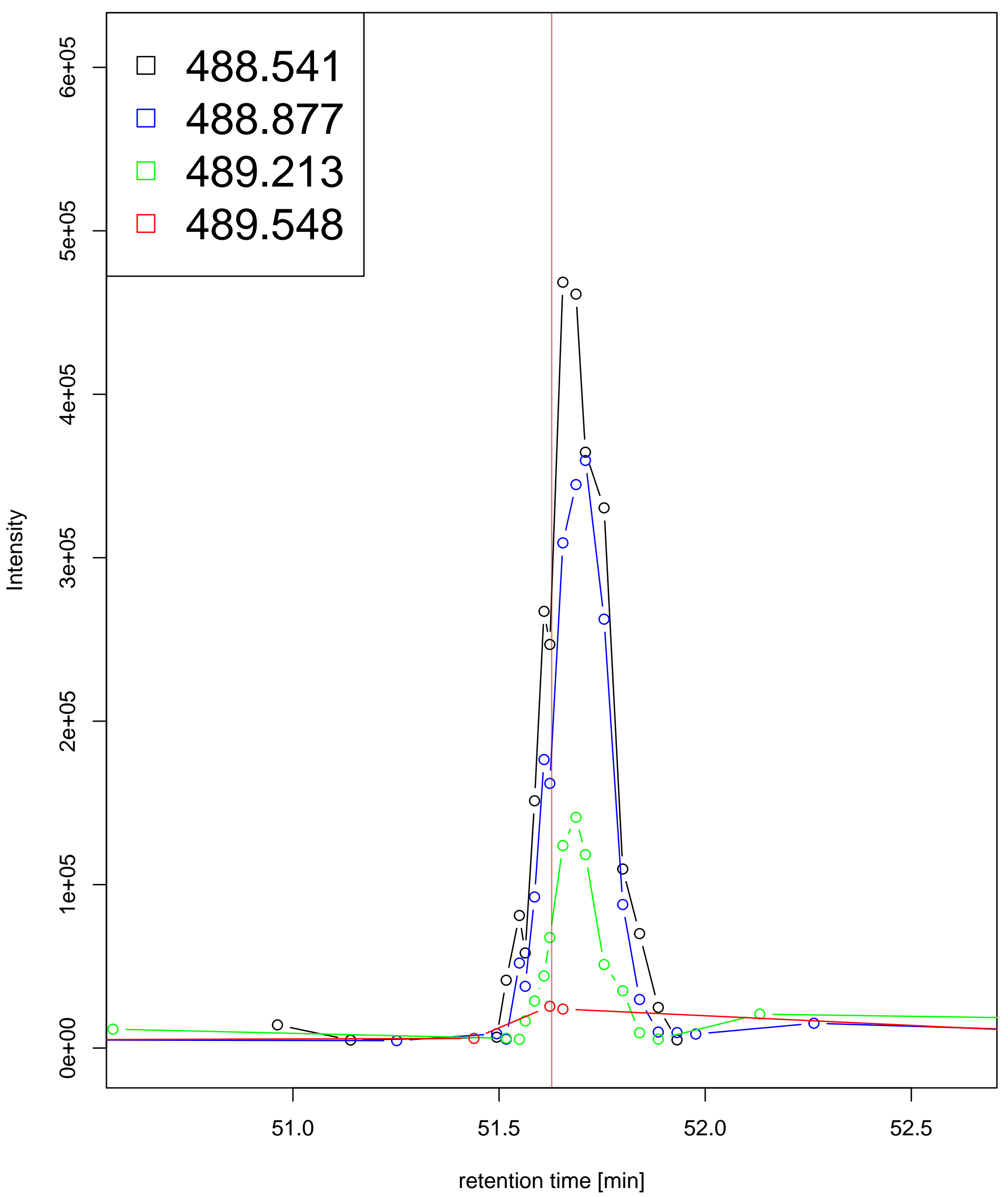

MS1 of ScanNo:8960

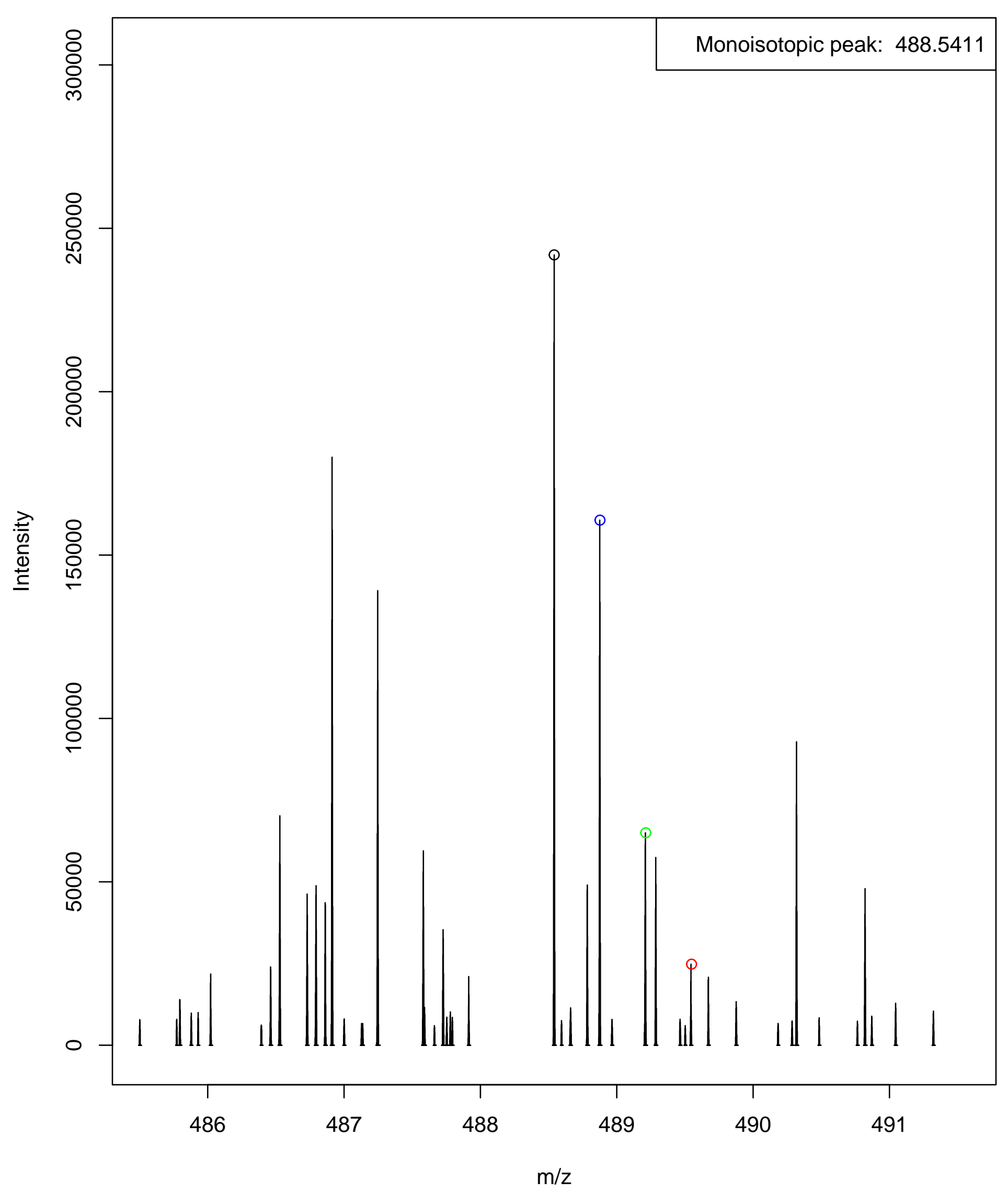
charge $=3$ 
XIC of MS1

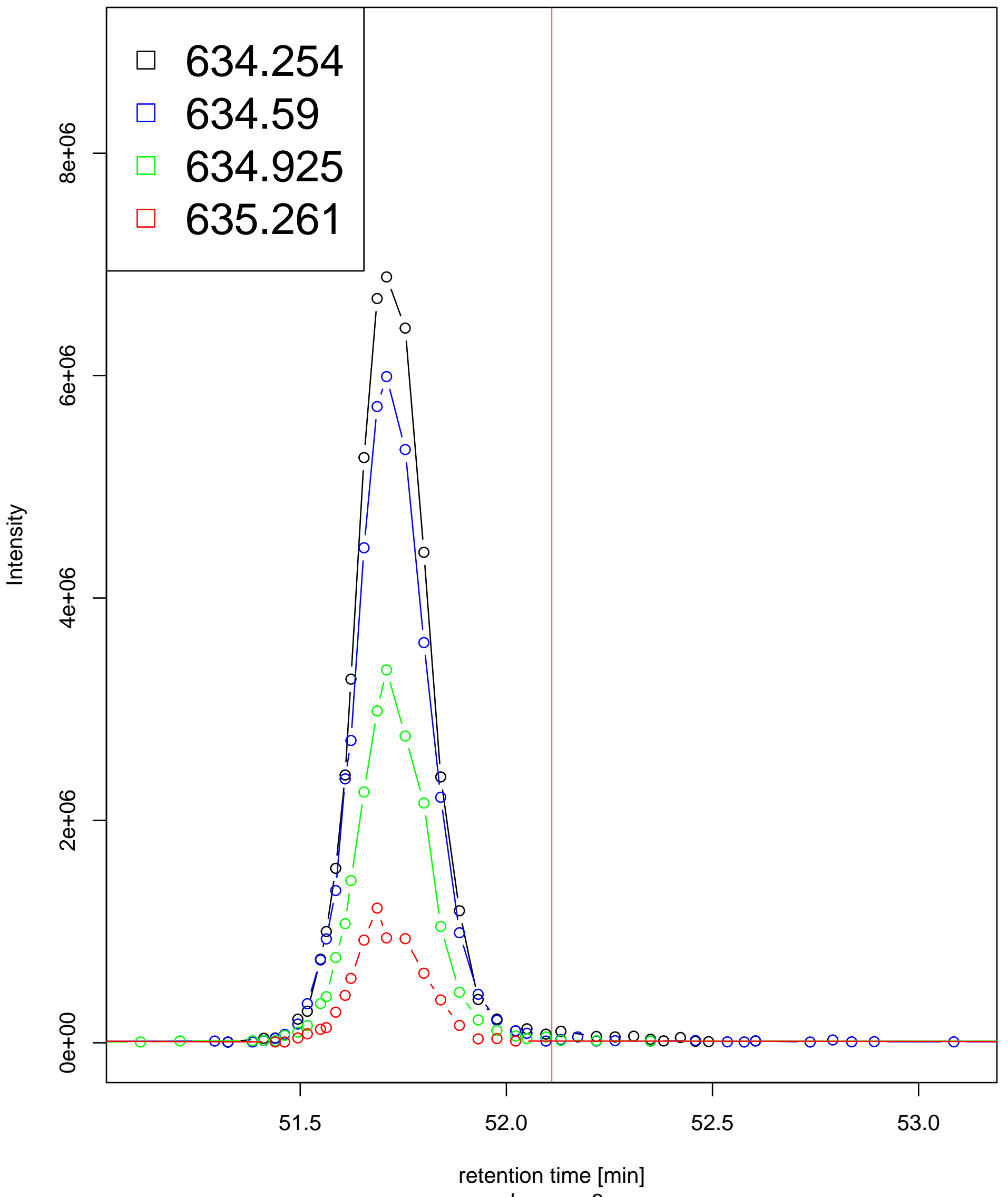

MS1 of ScanNo:9063

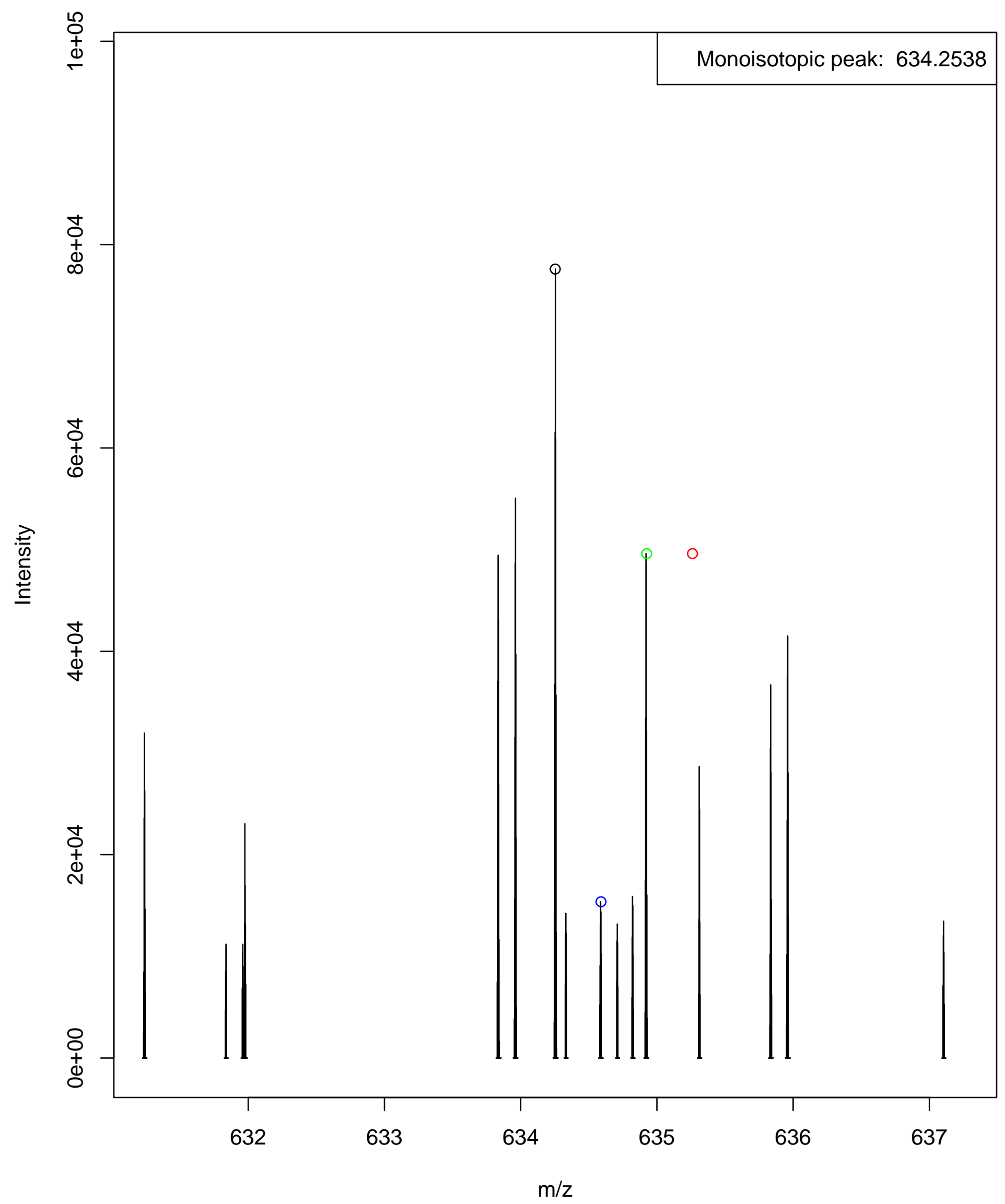

charge $=3$ 
XIC of MS1

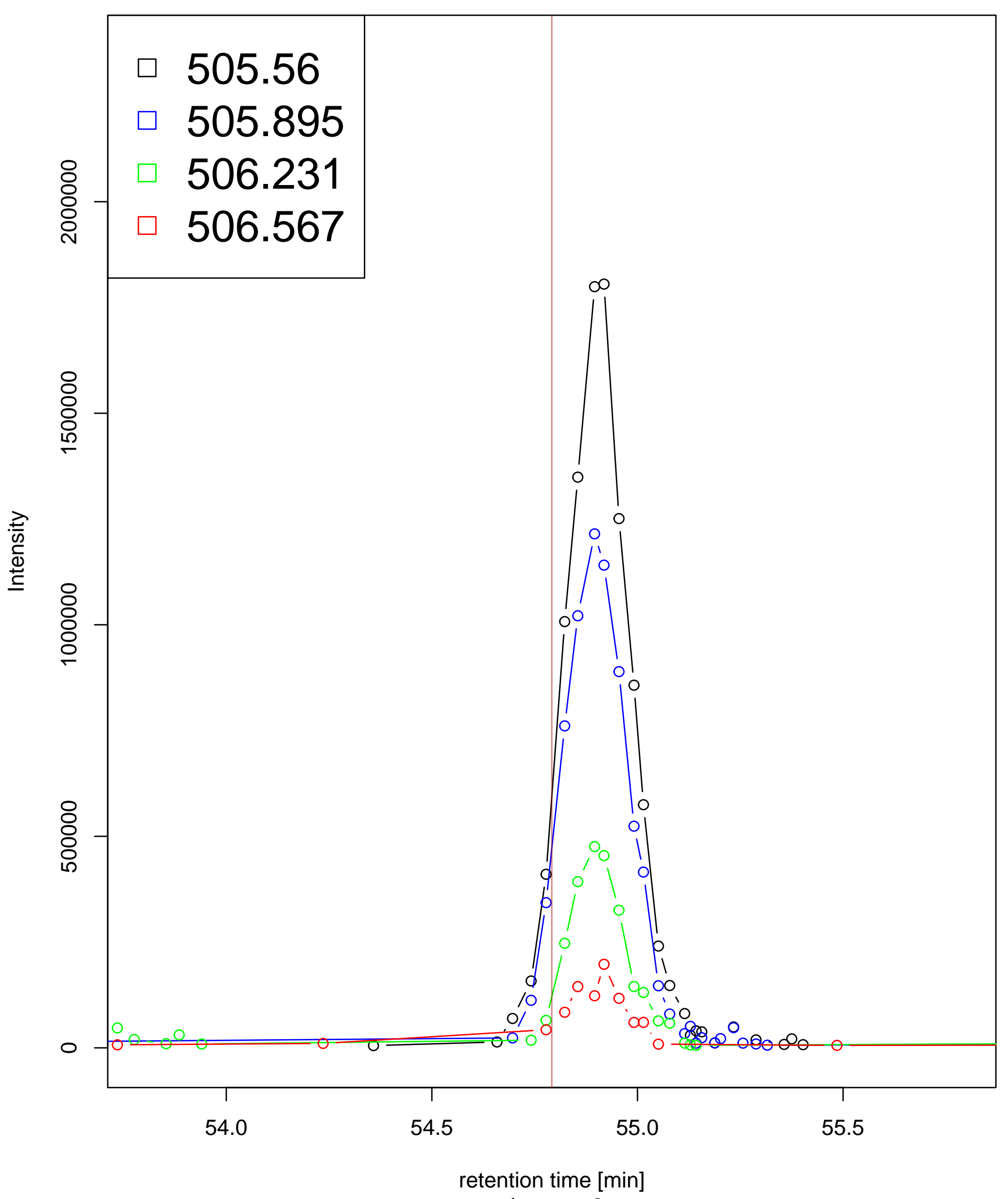

MS1 of ScanNo:9650

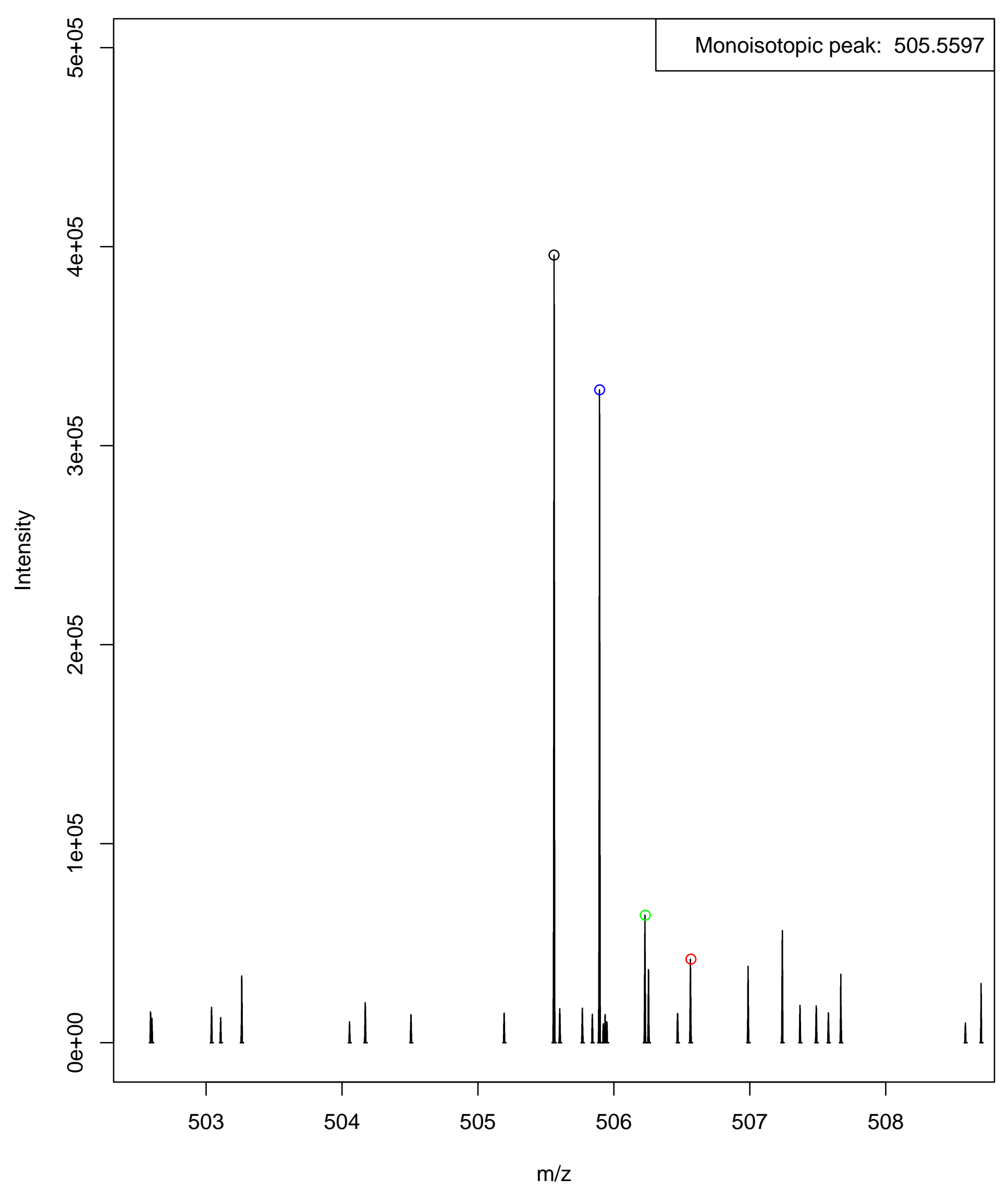


XIC of MS1

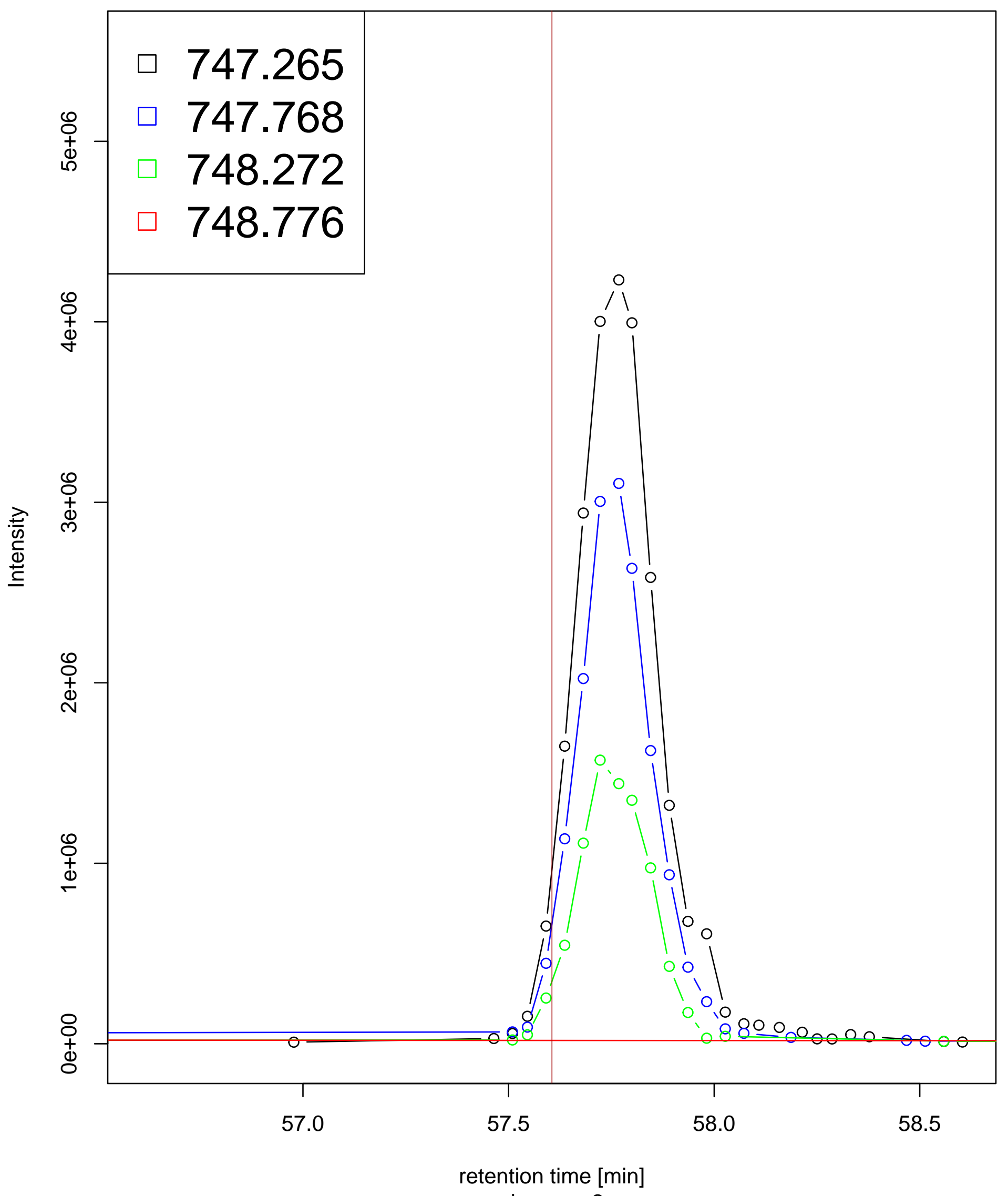

MS1 of ScanNo:10265

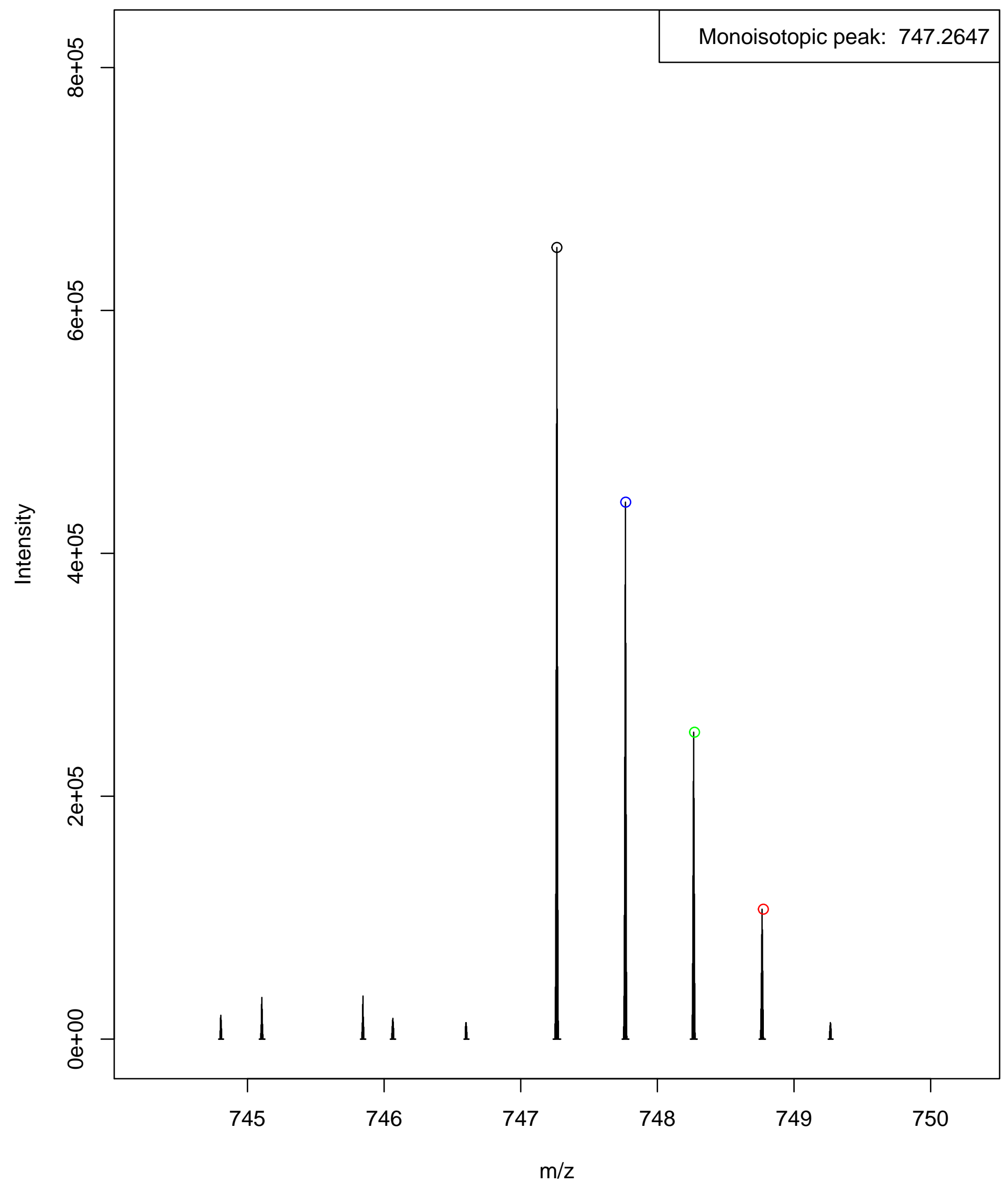


XIC of MS1

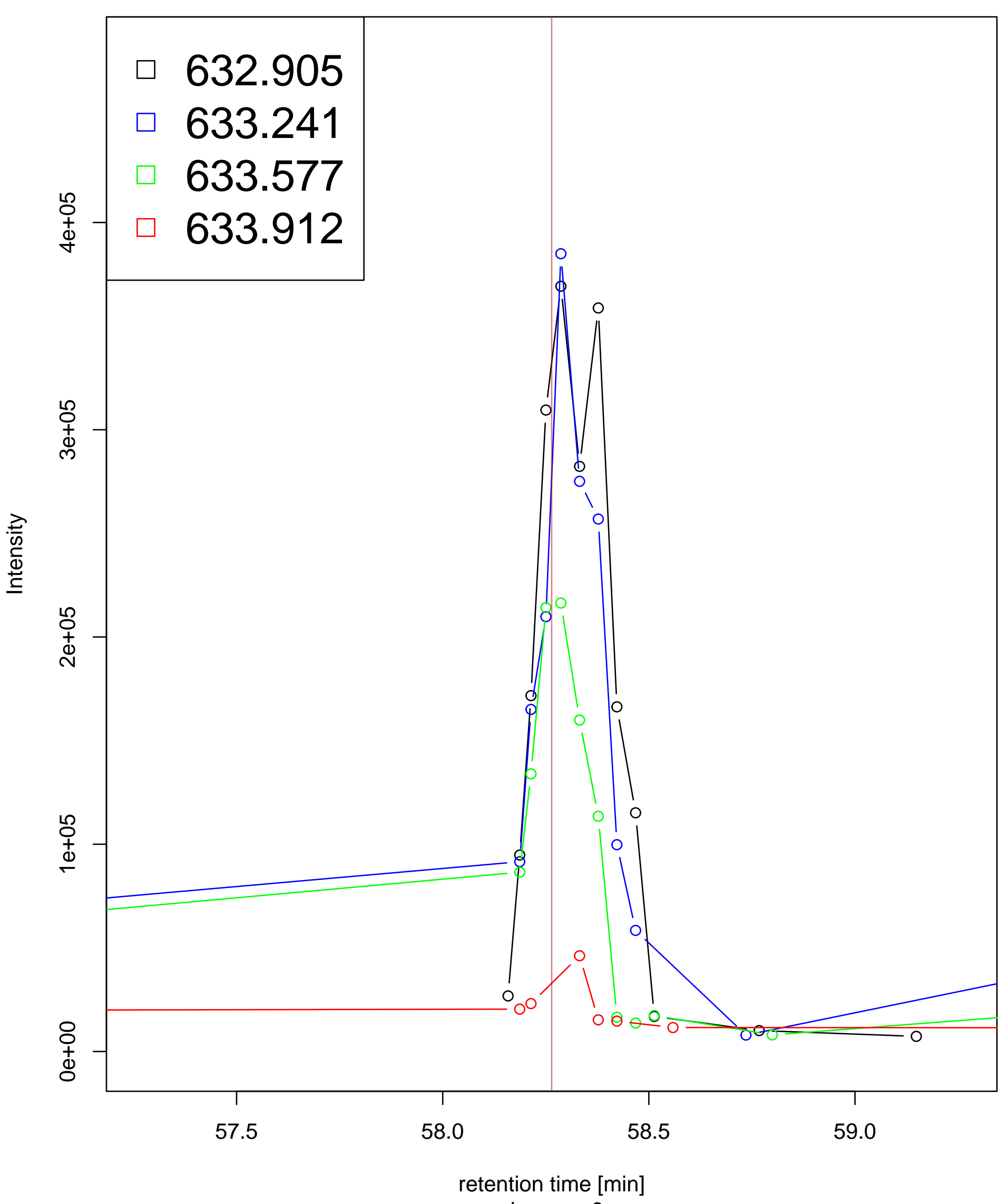

MS1 of ScanNo:10410

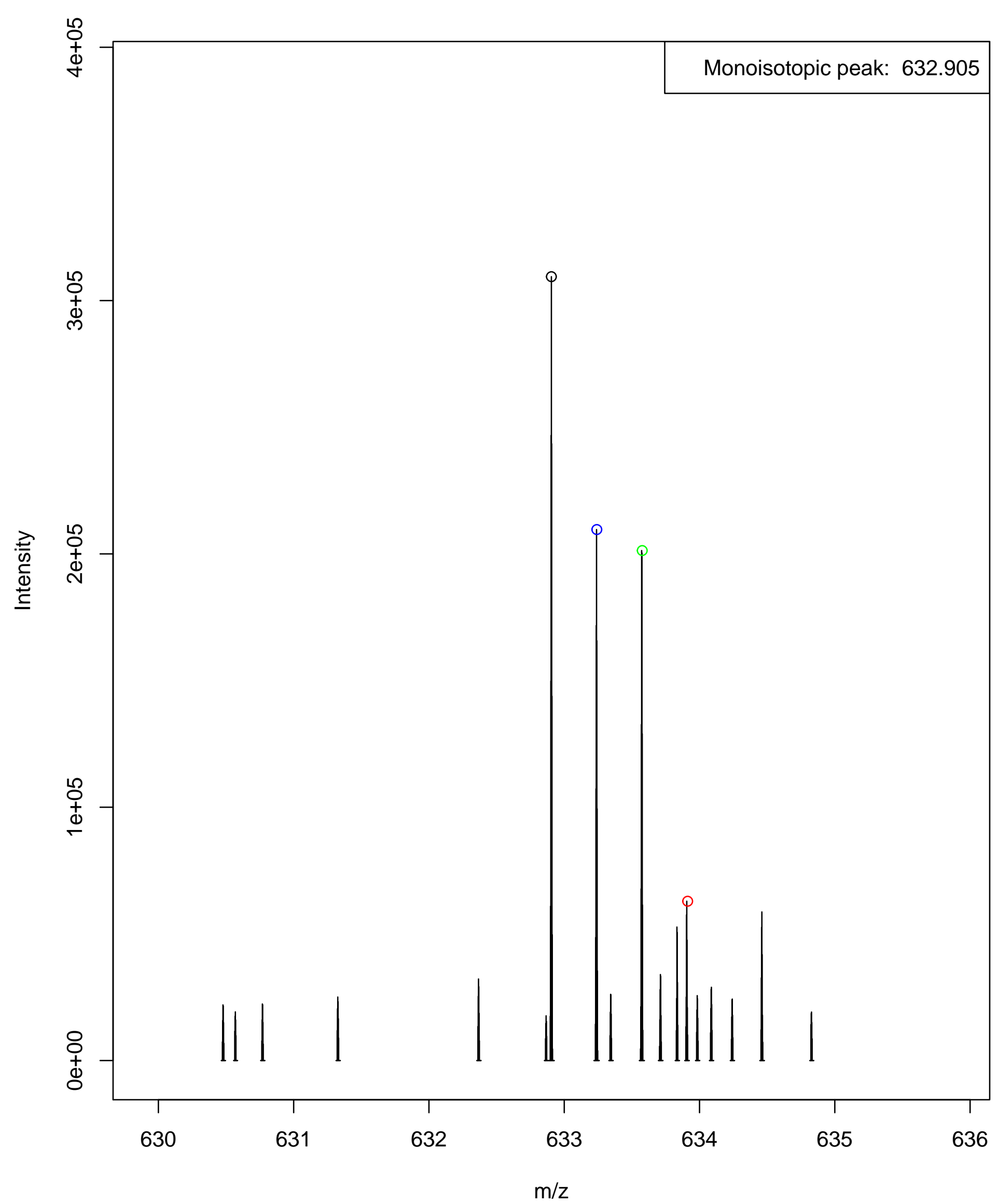


XIC of MS1

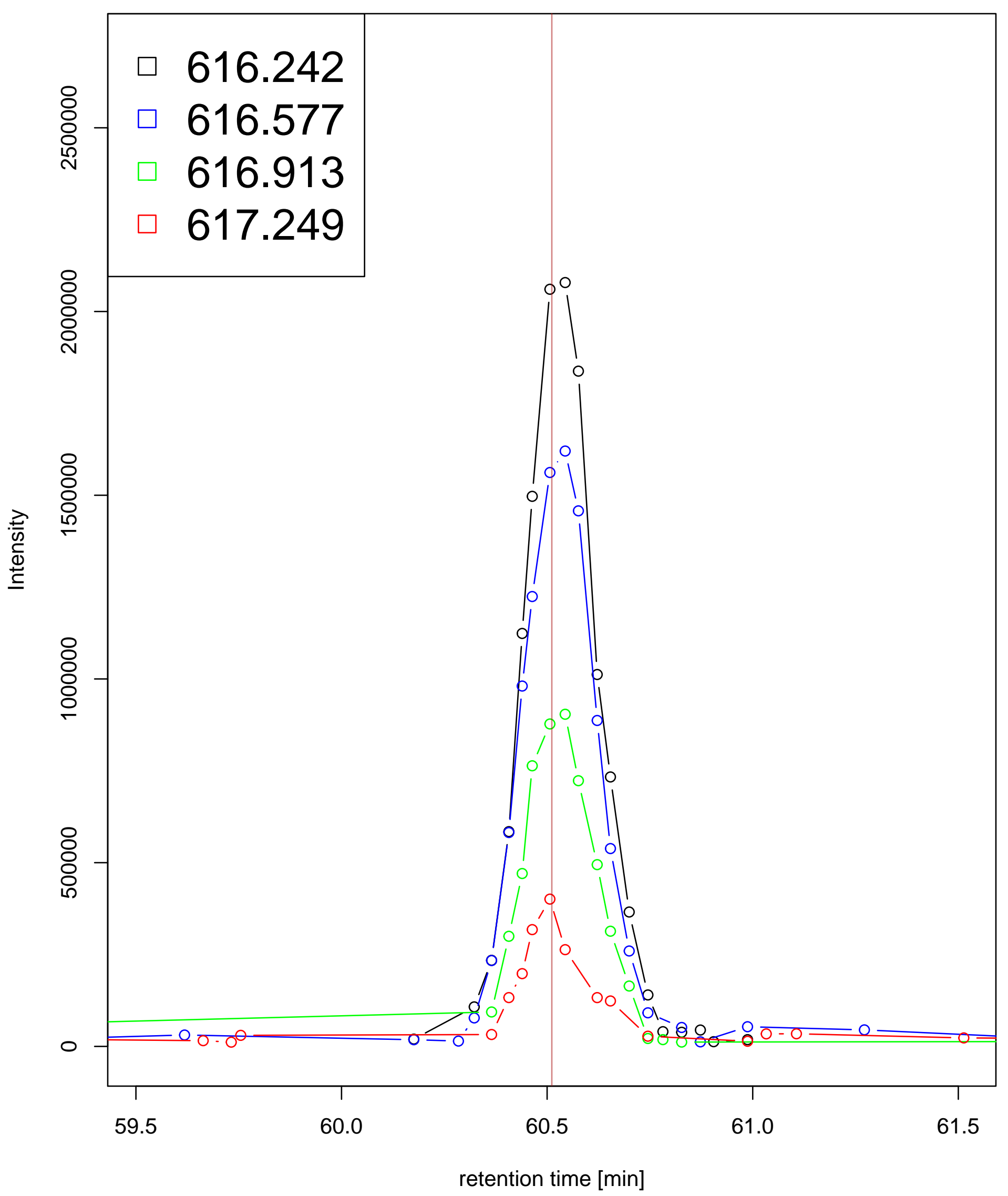

MS1 of ScanNo:10891

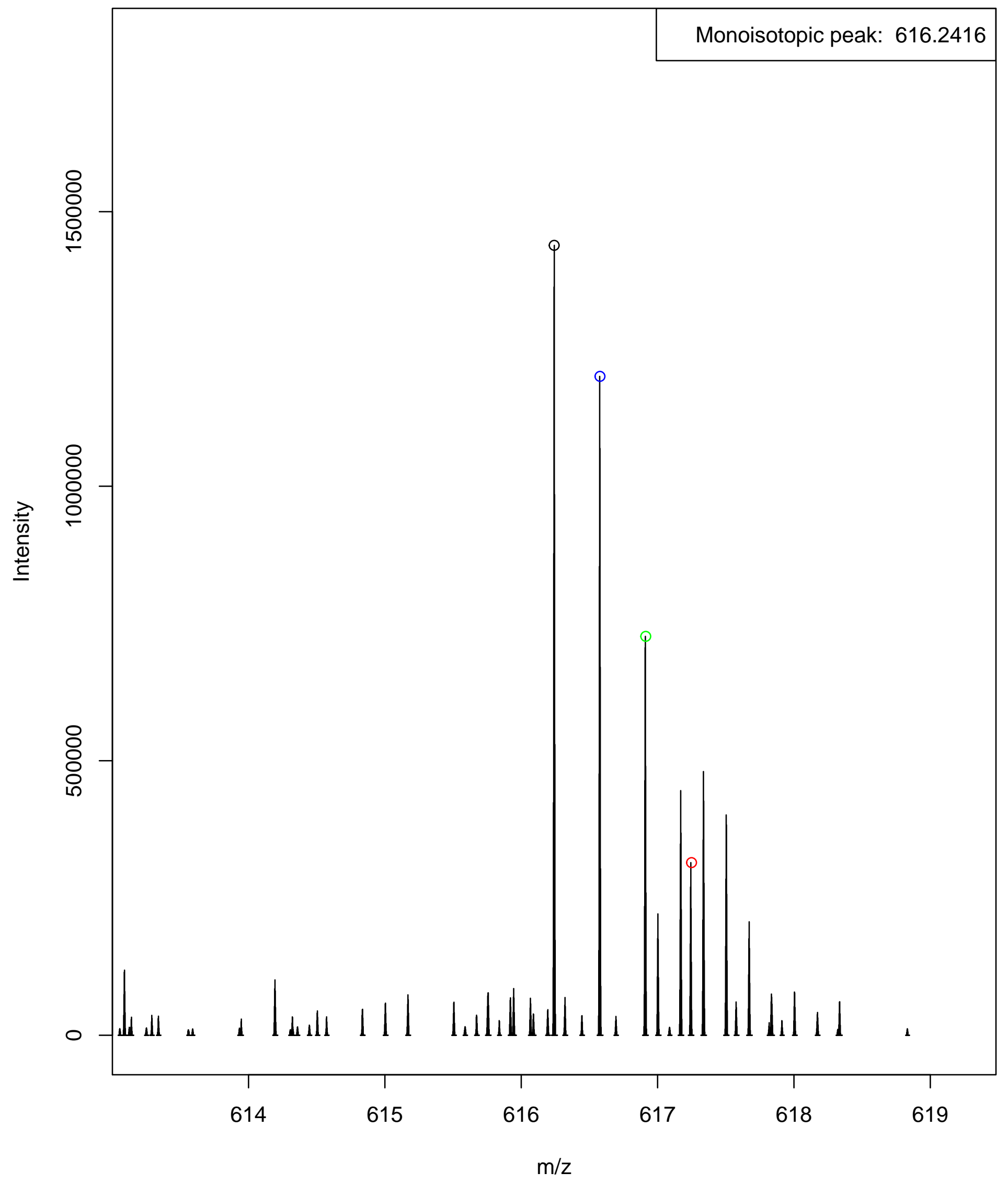


XIC of MS1

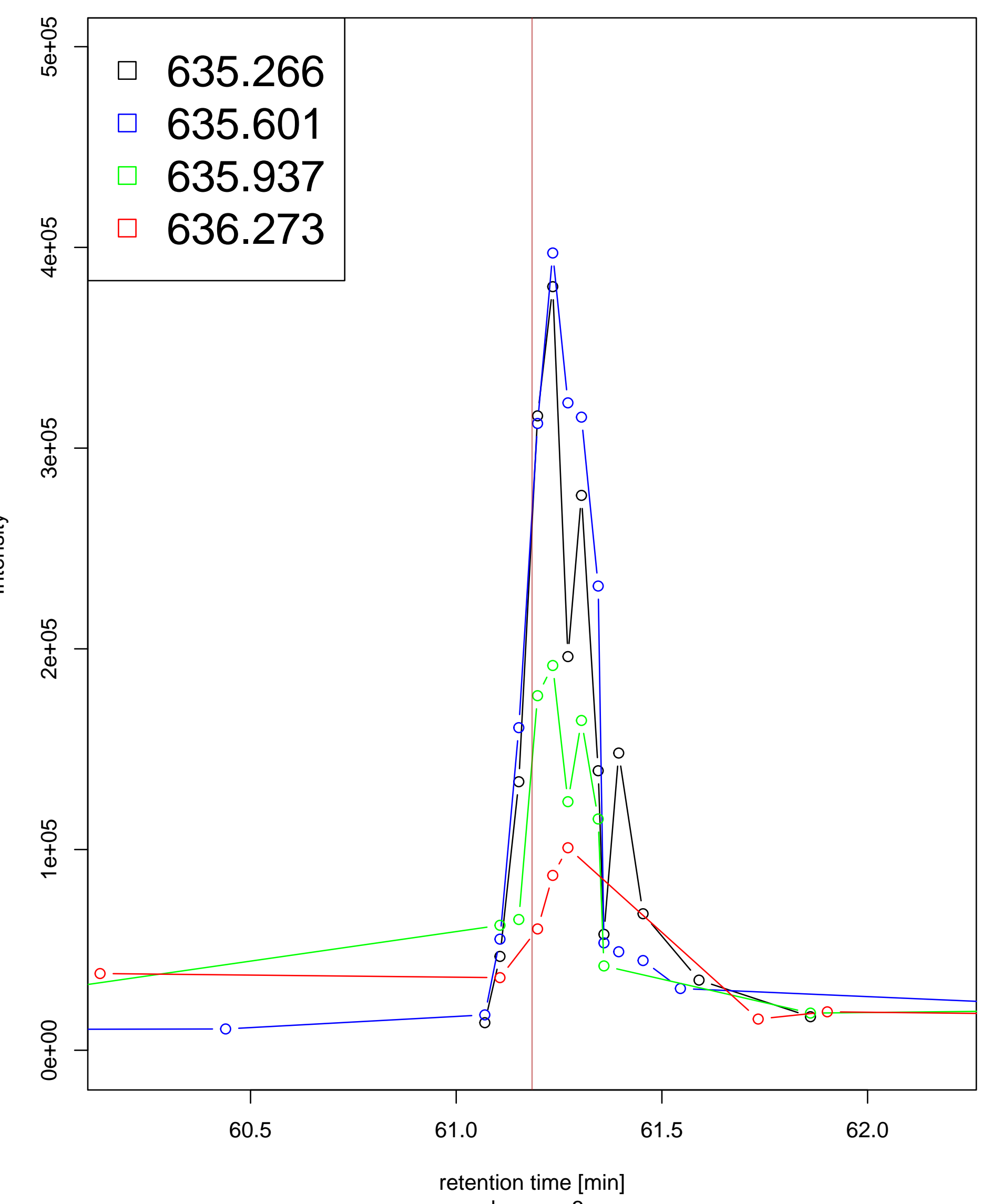

MS1 of ScanNo:11050

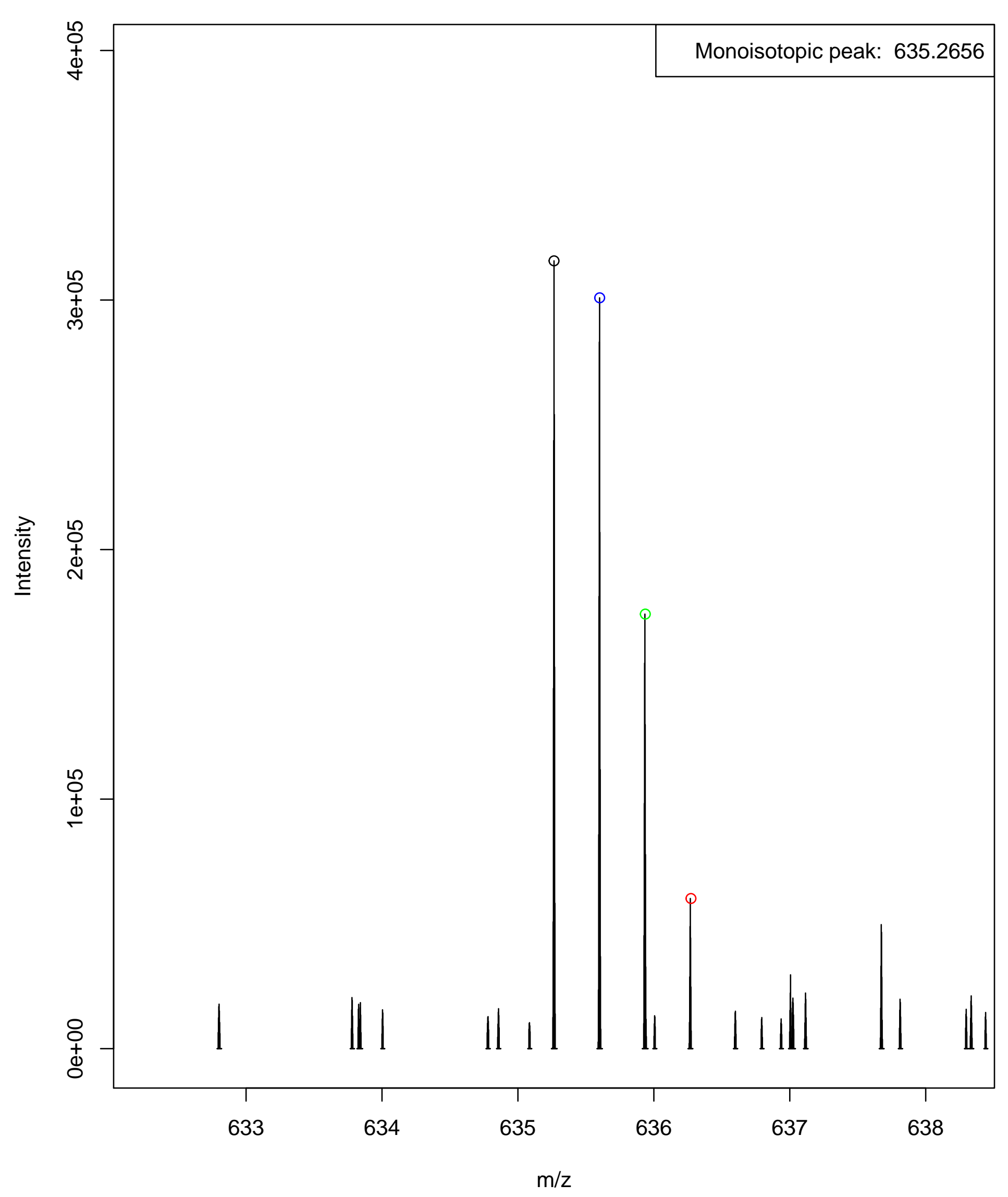

charge $=3$ 
XIC of MS1

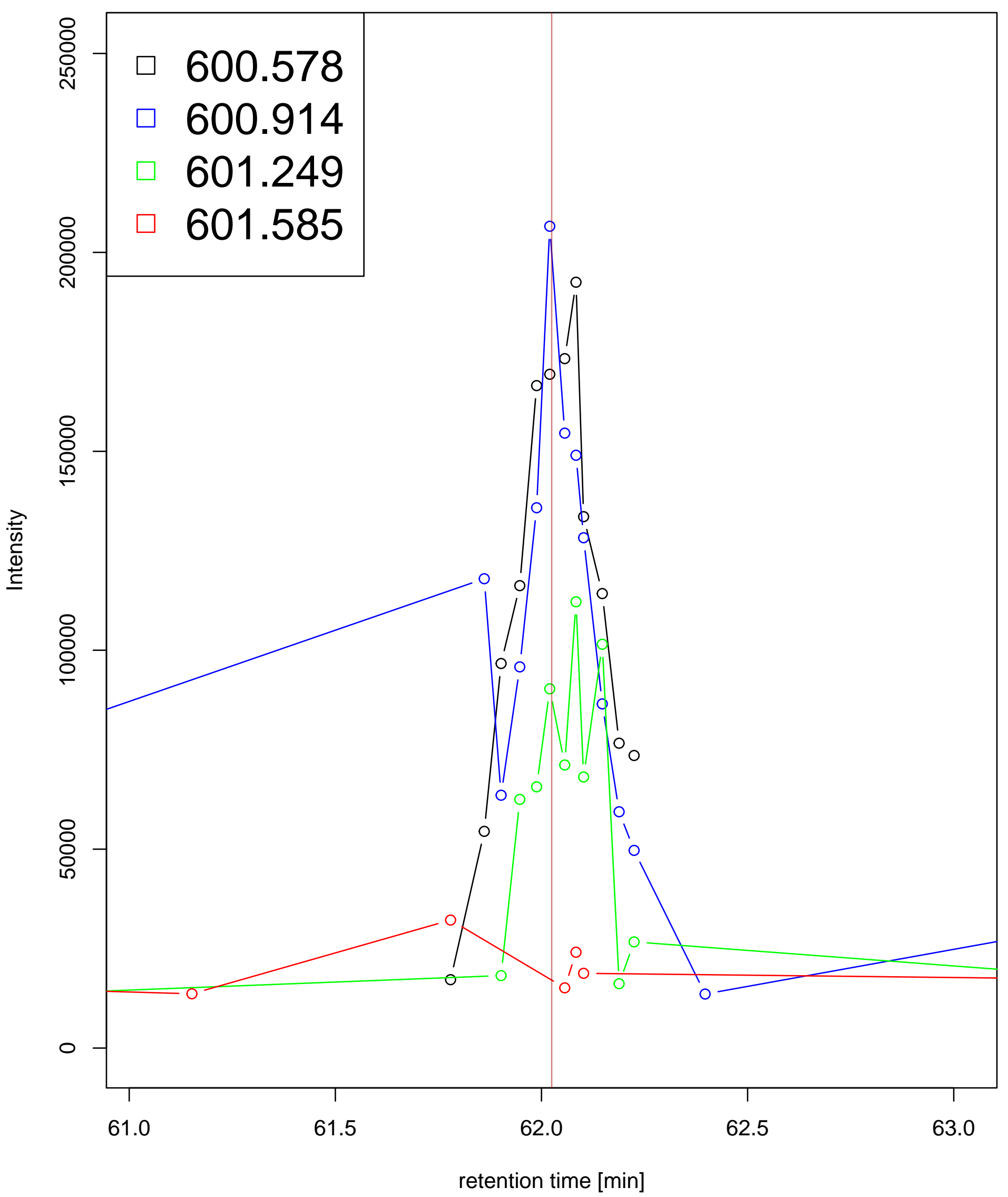

MS1 of ScanNo:11223

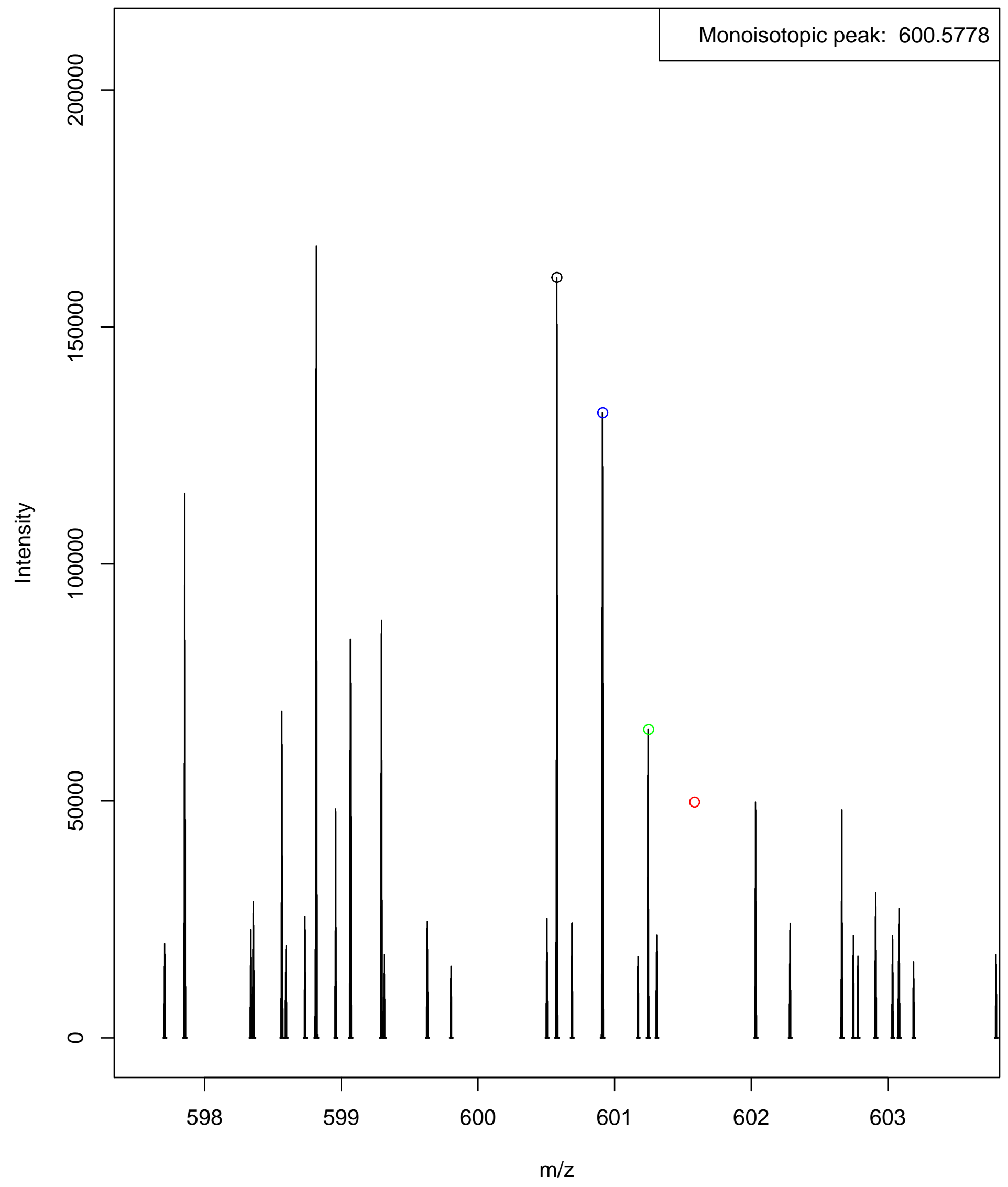

charge $=3$ 
XIC of MS1

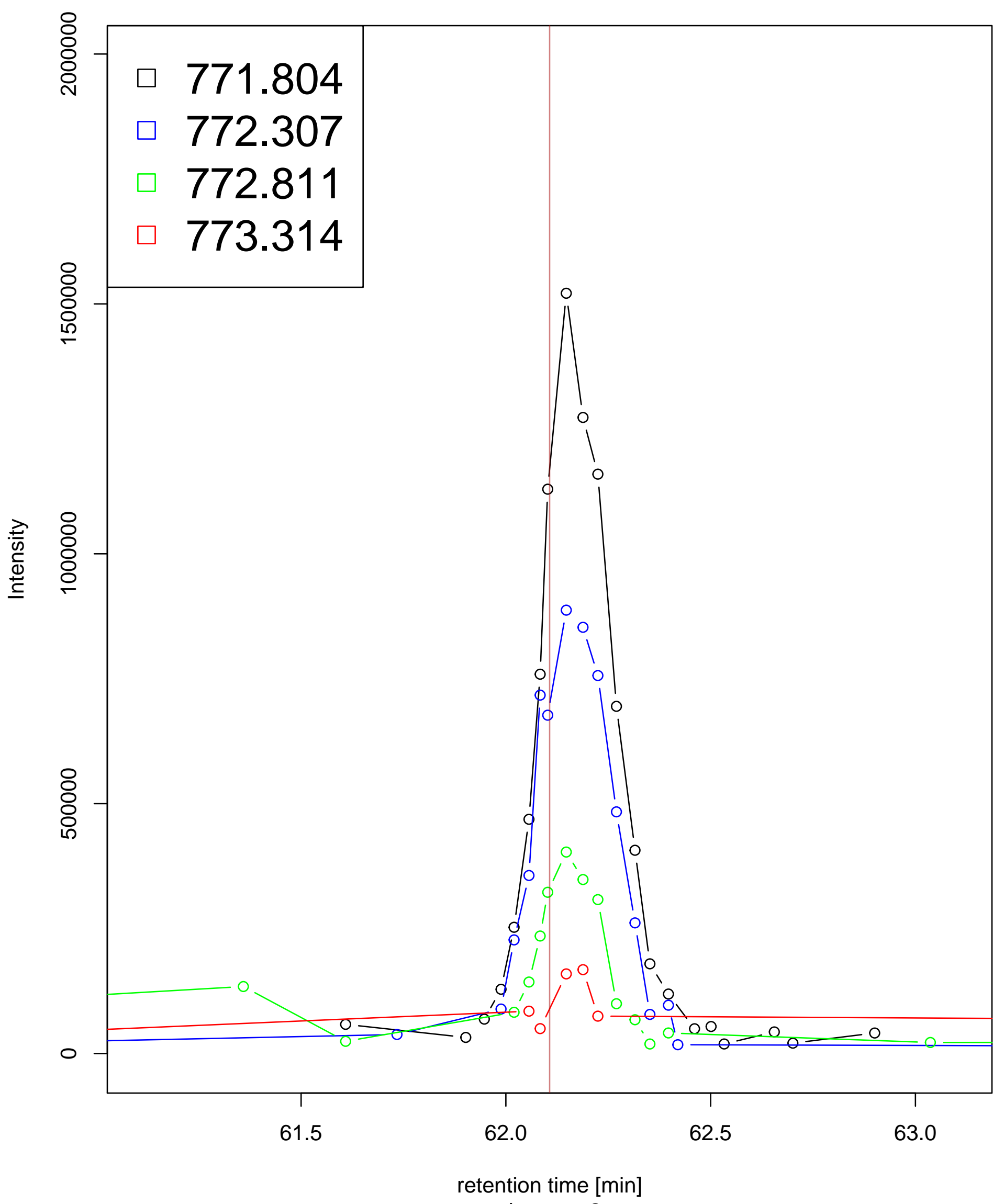

MS1 of ScanNo:11244

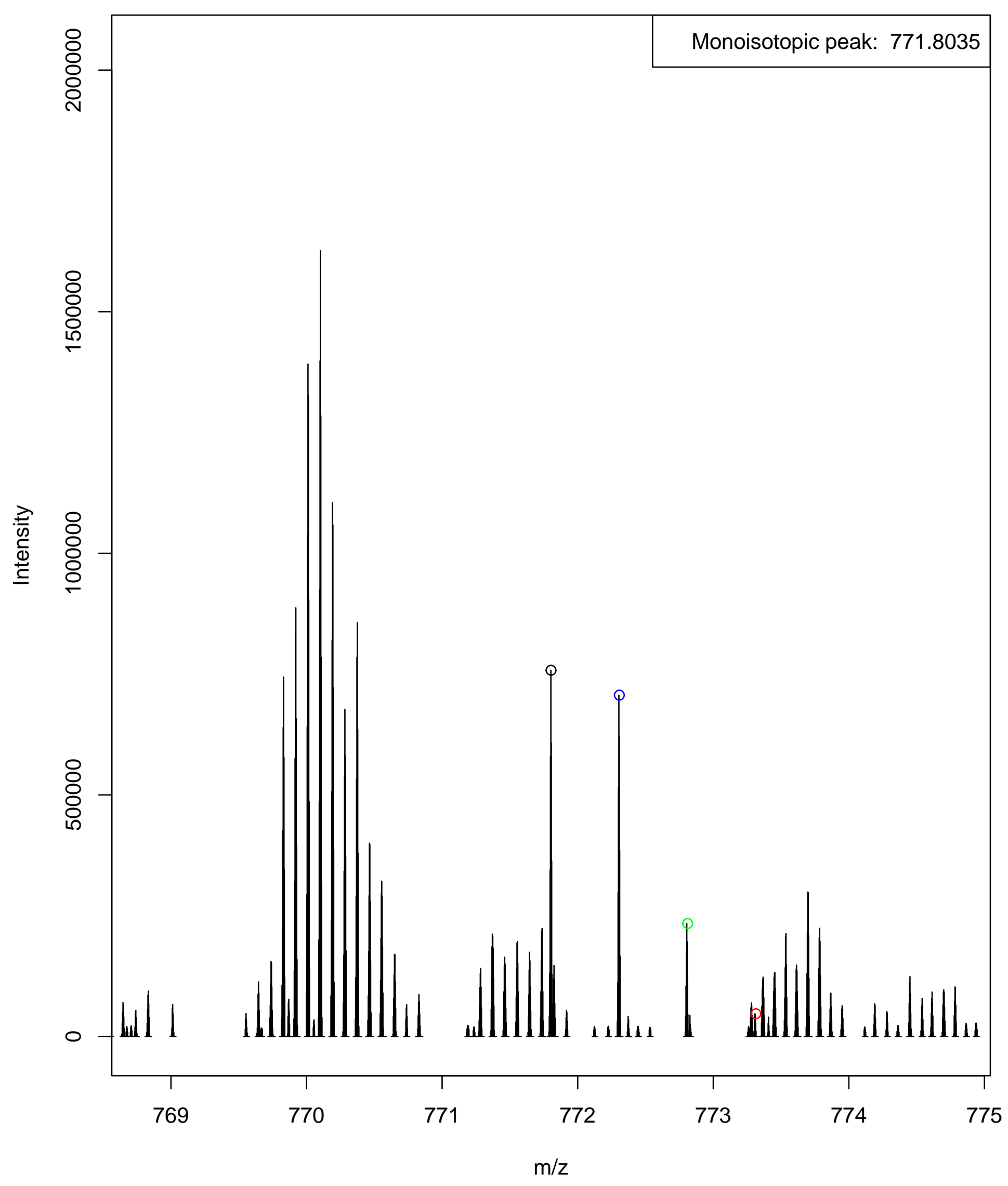


XIC of MS1

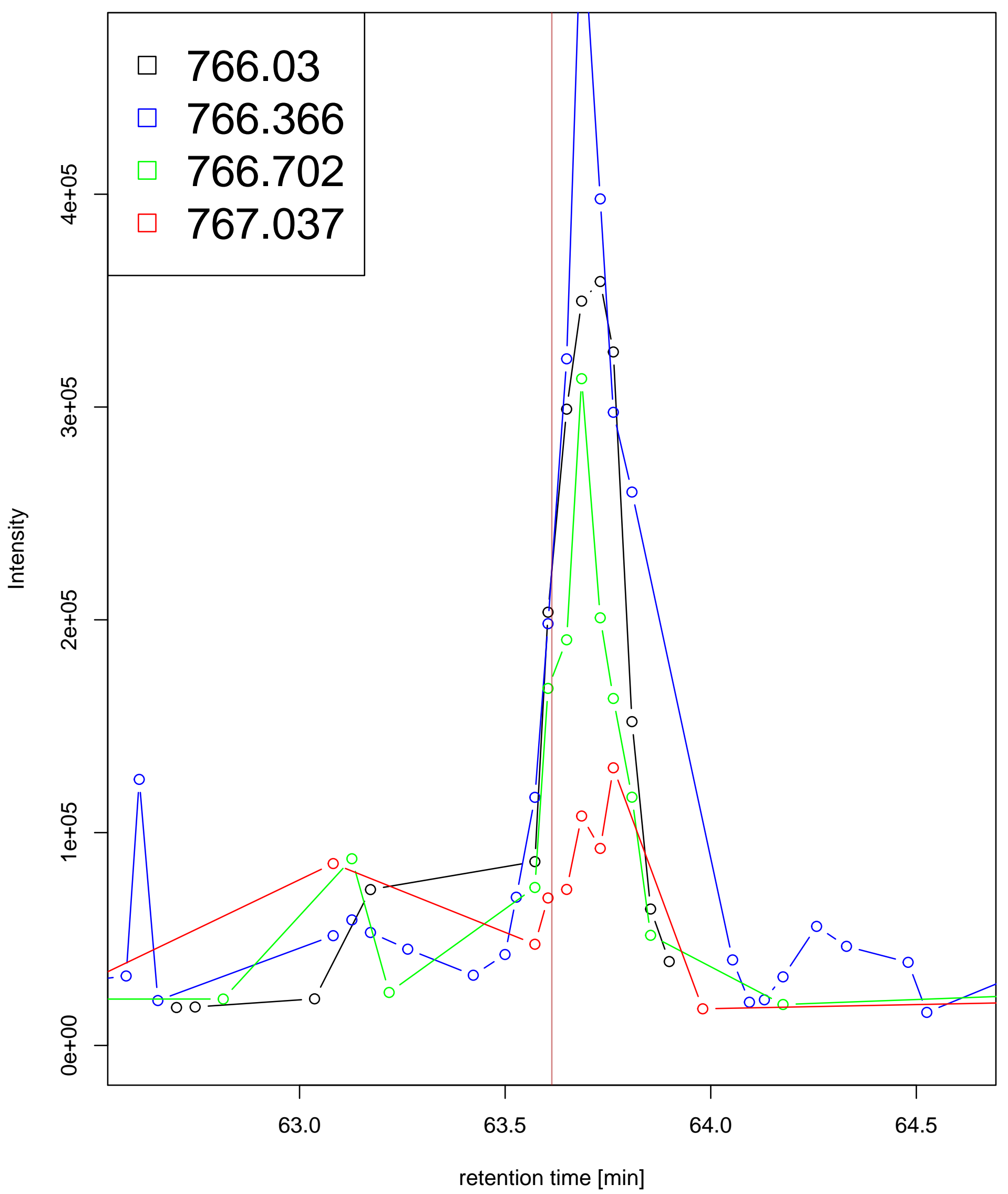

MS1 of ScanNo:11571

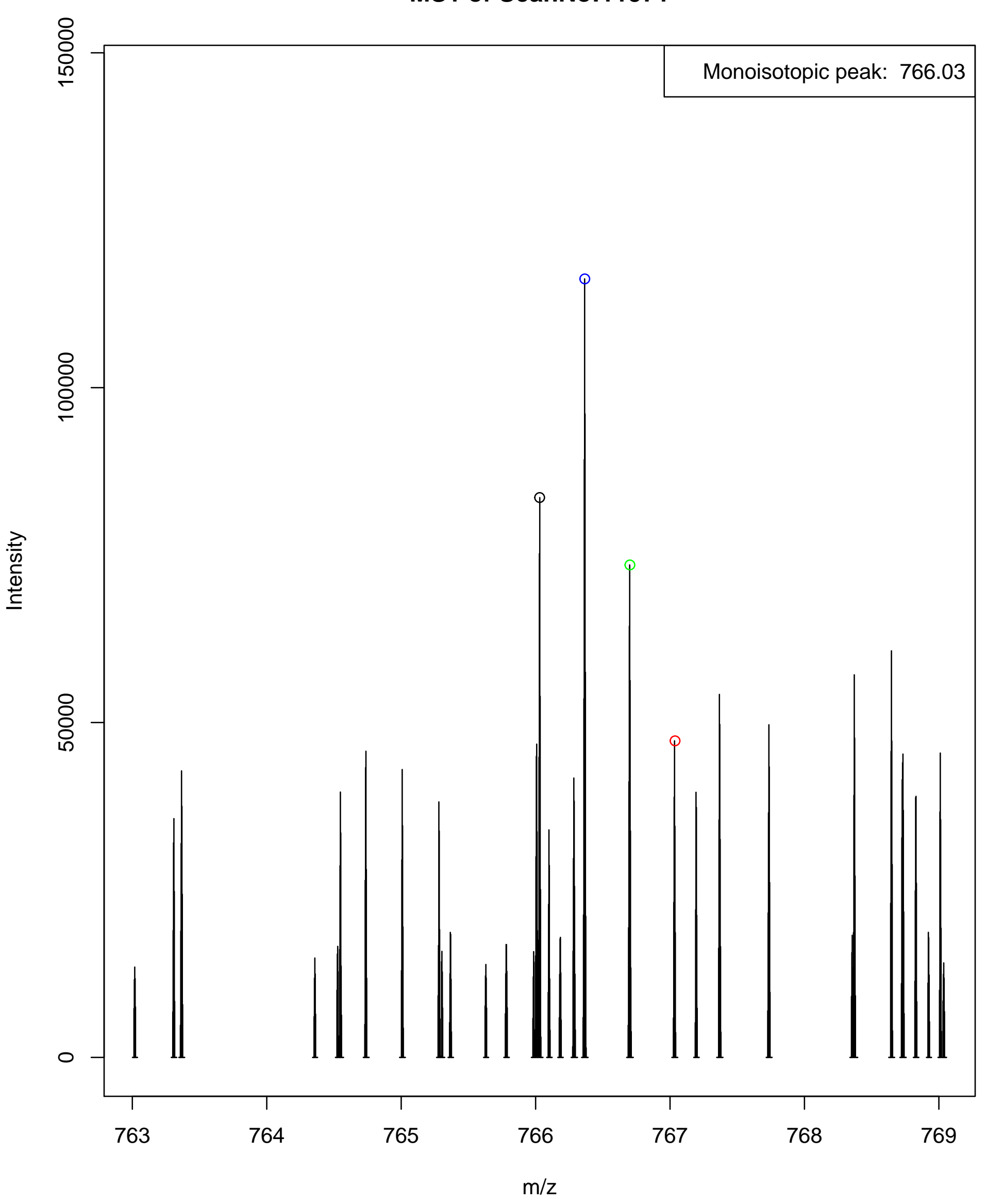

charge $=3$ 


\section{XIC of MS1}

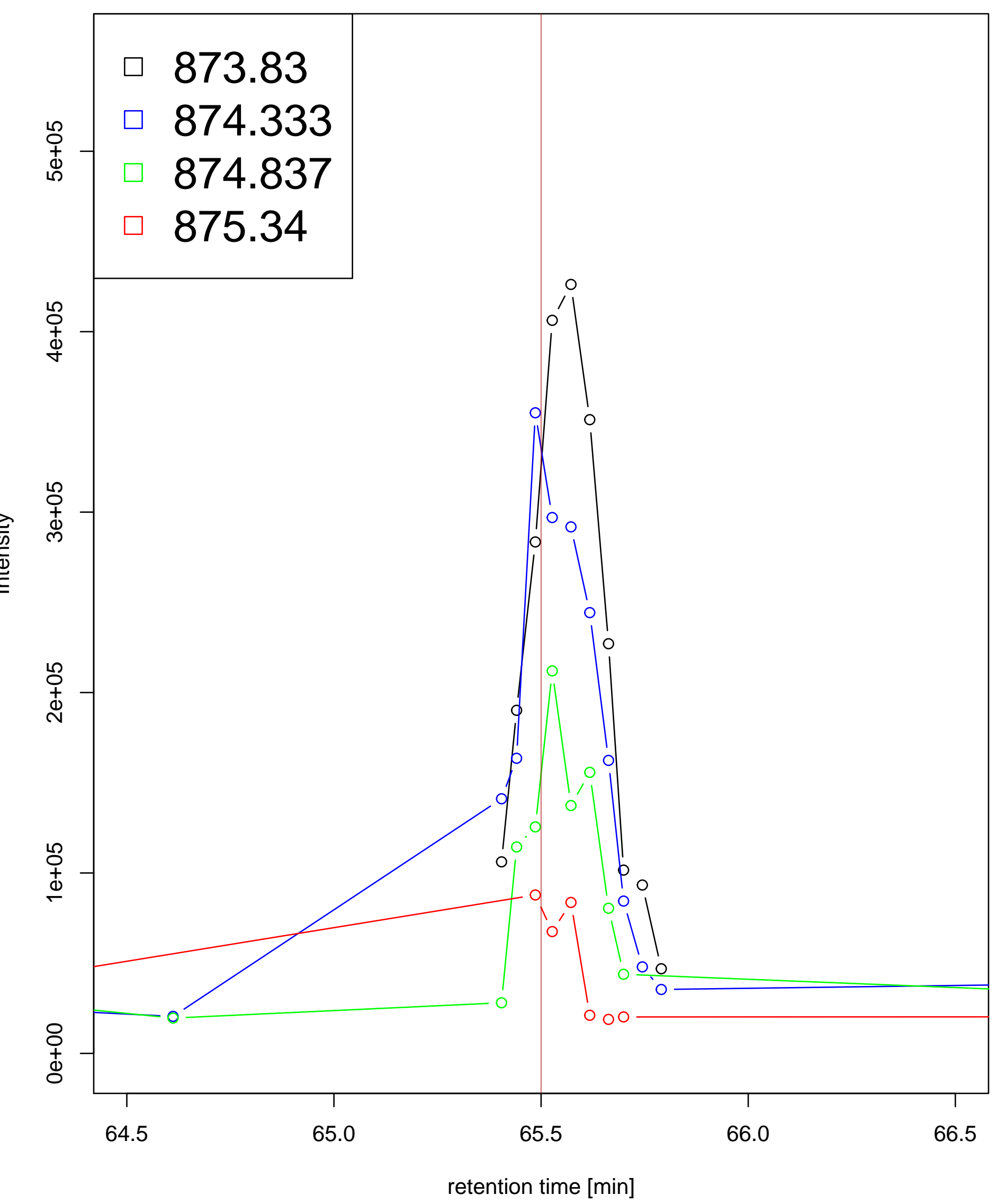

MS1 of ScanNo:11992

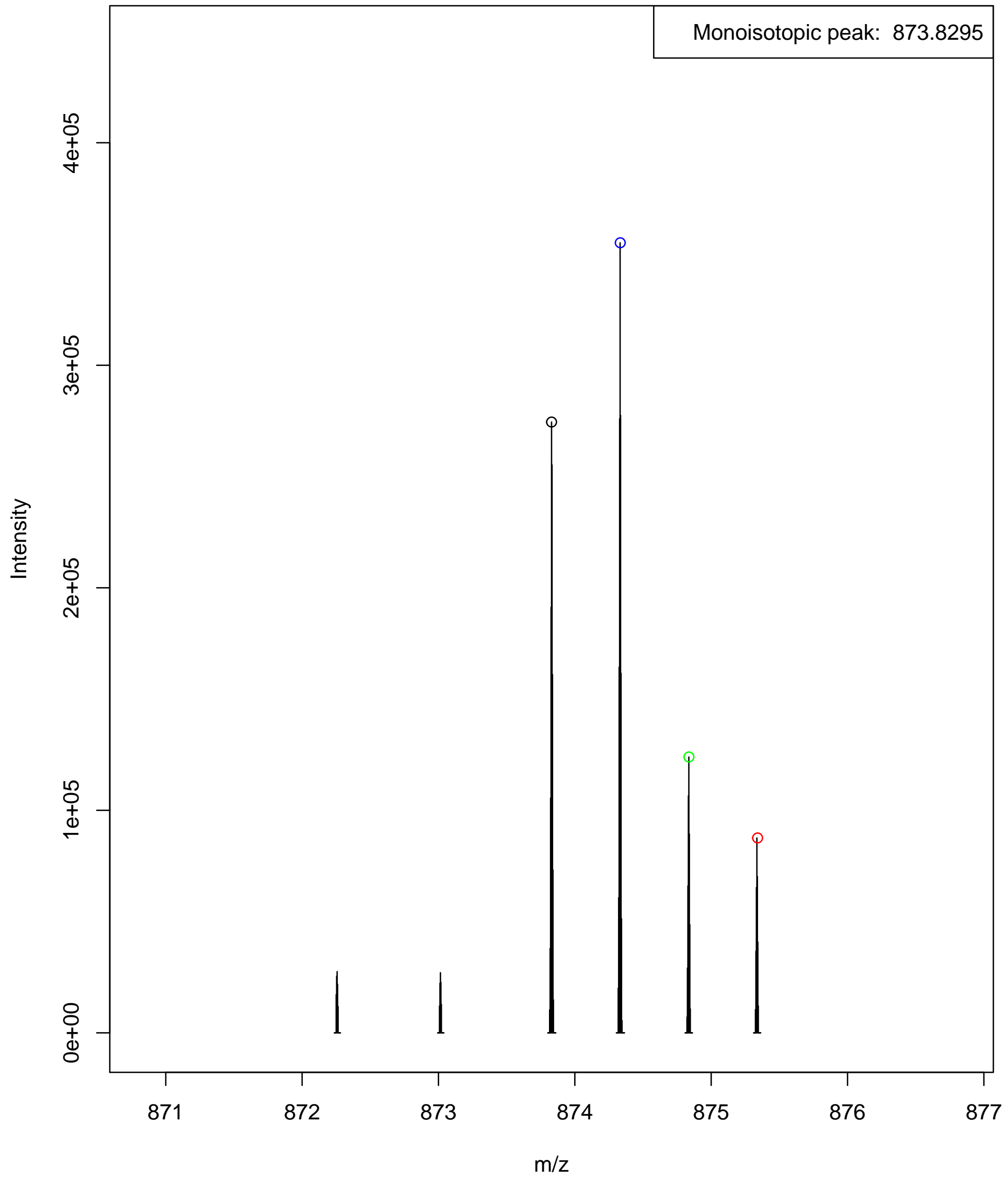


XIC of MS1

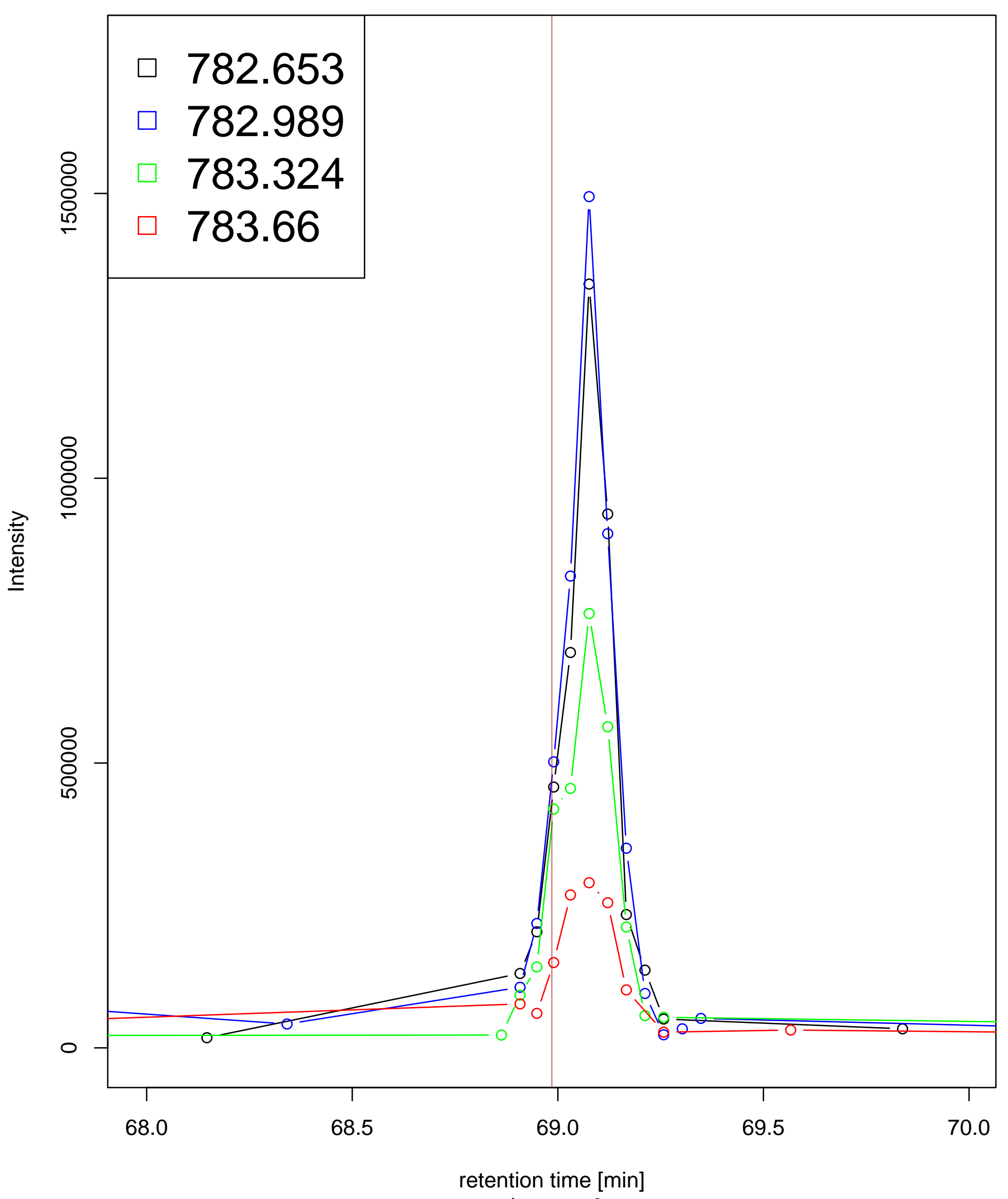

MS1 of ScanNo:12761

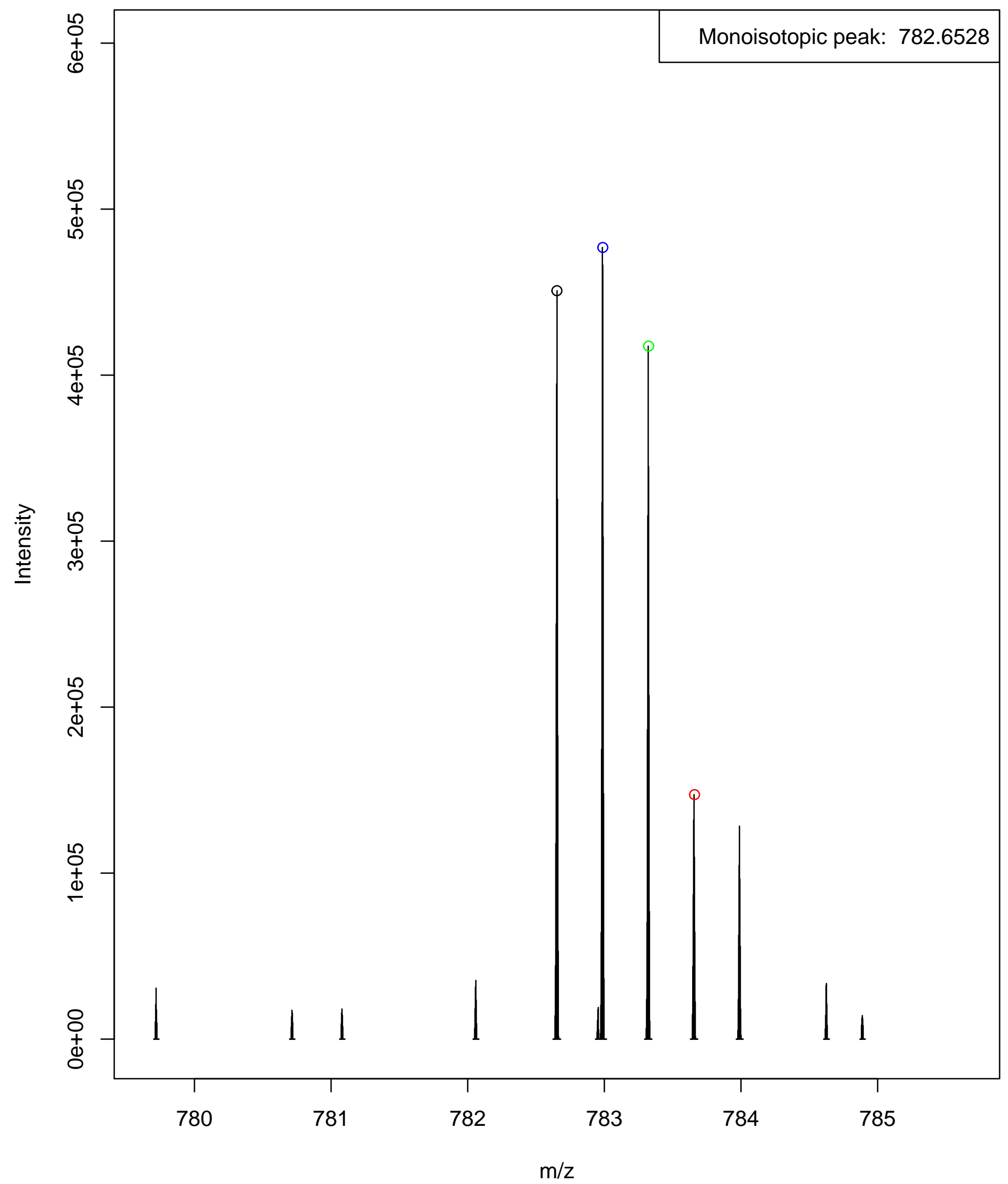


XIC of MS1

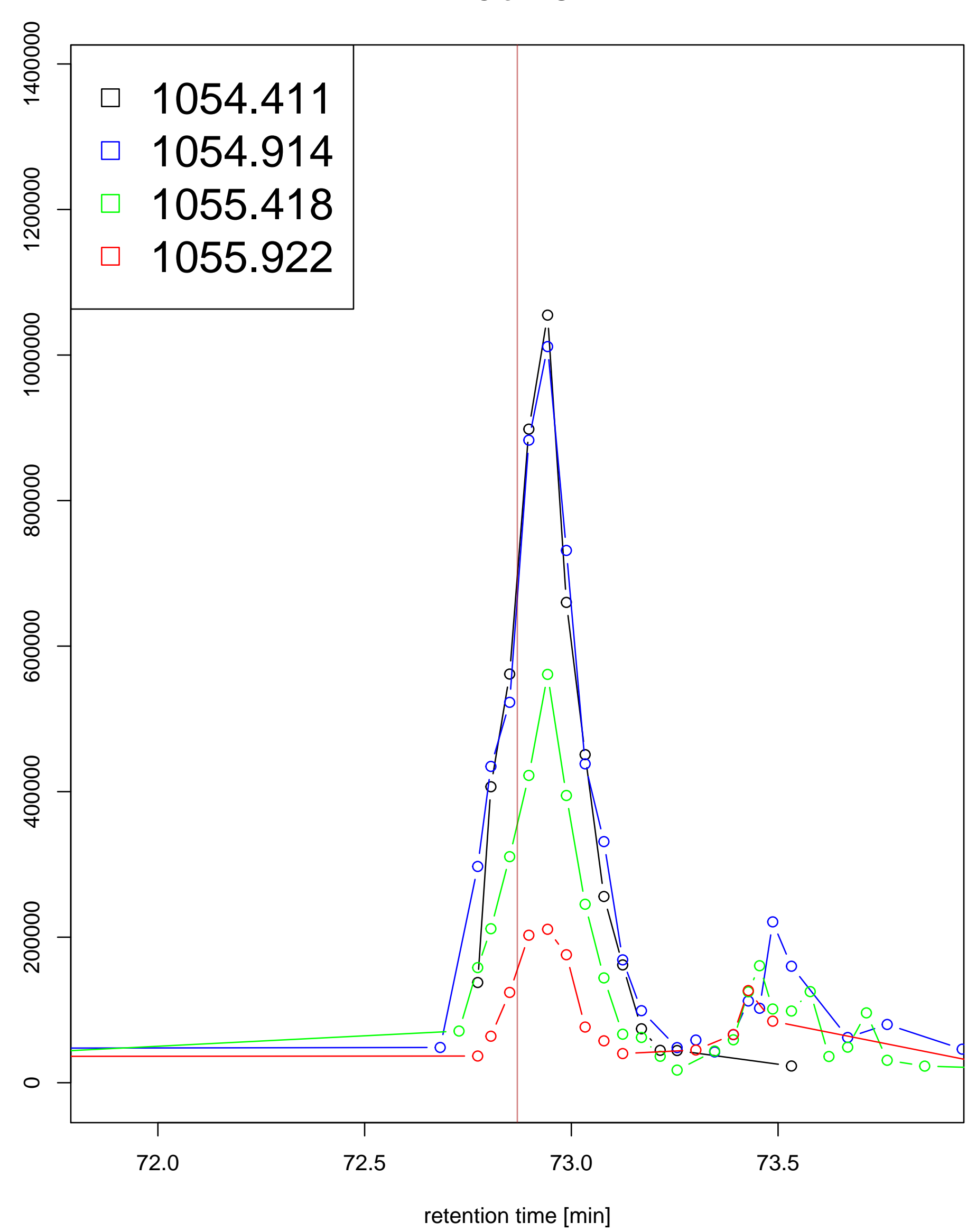

MS1 of ScanNo:13610

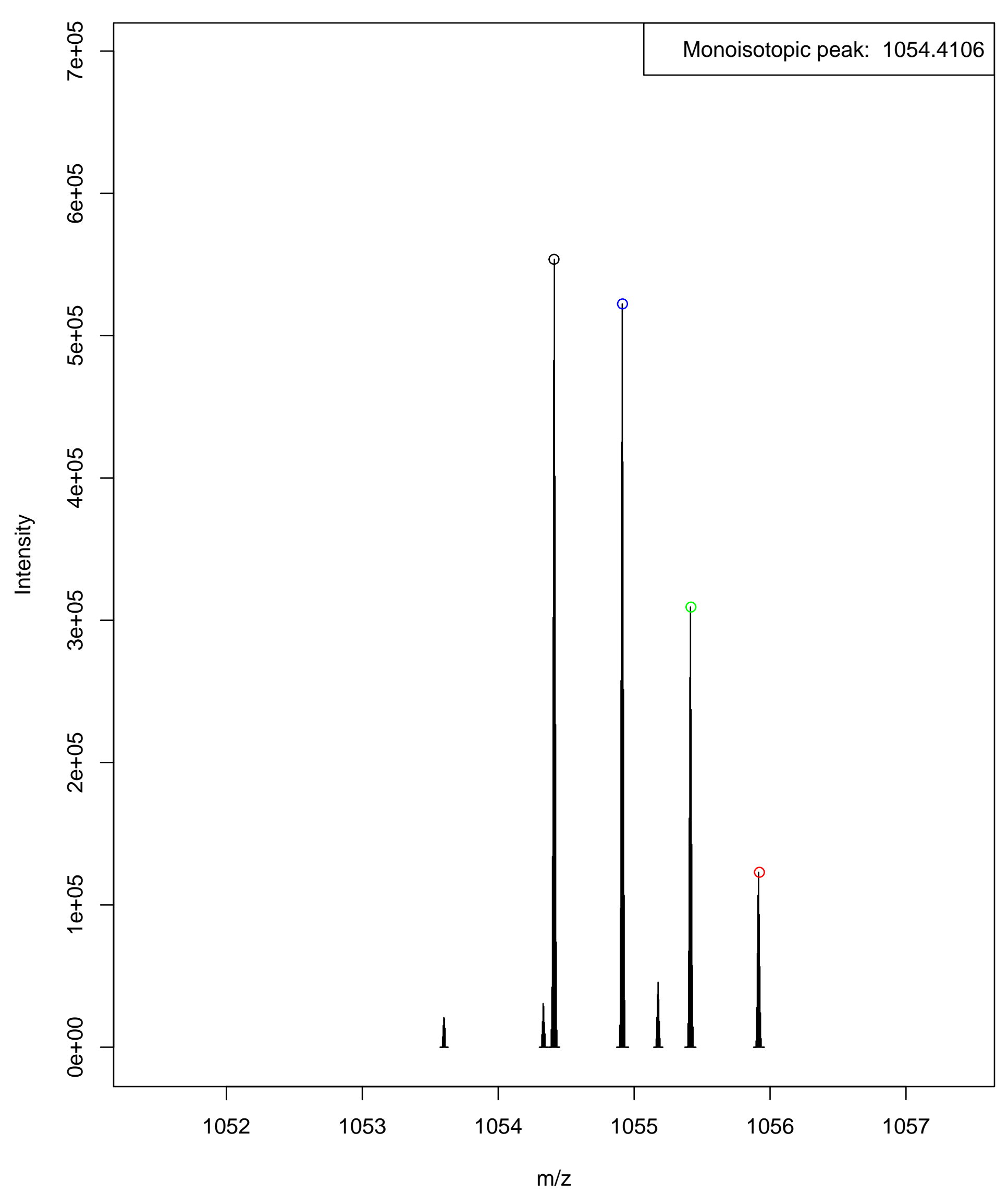


XIC of MS1

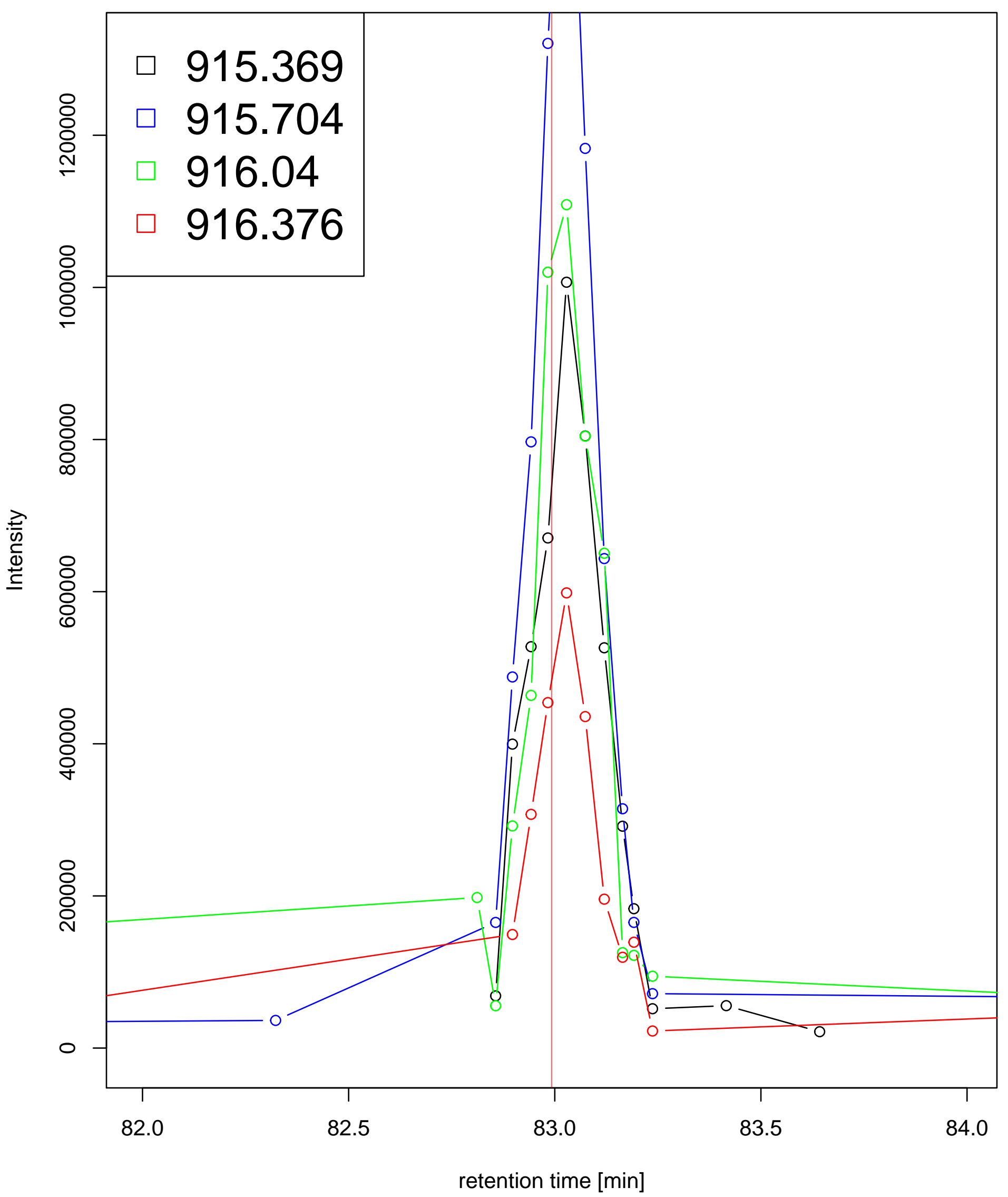

MS1 of ScanNo:15833

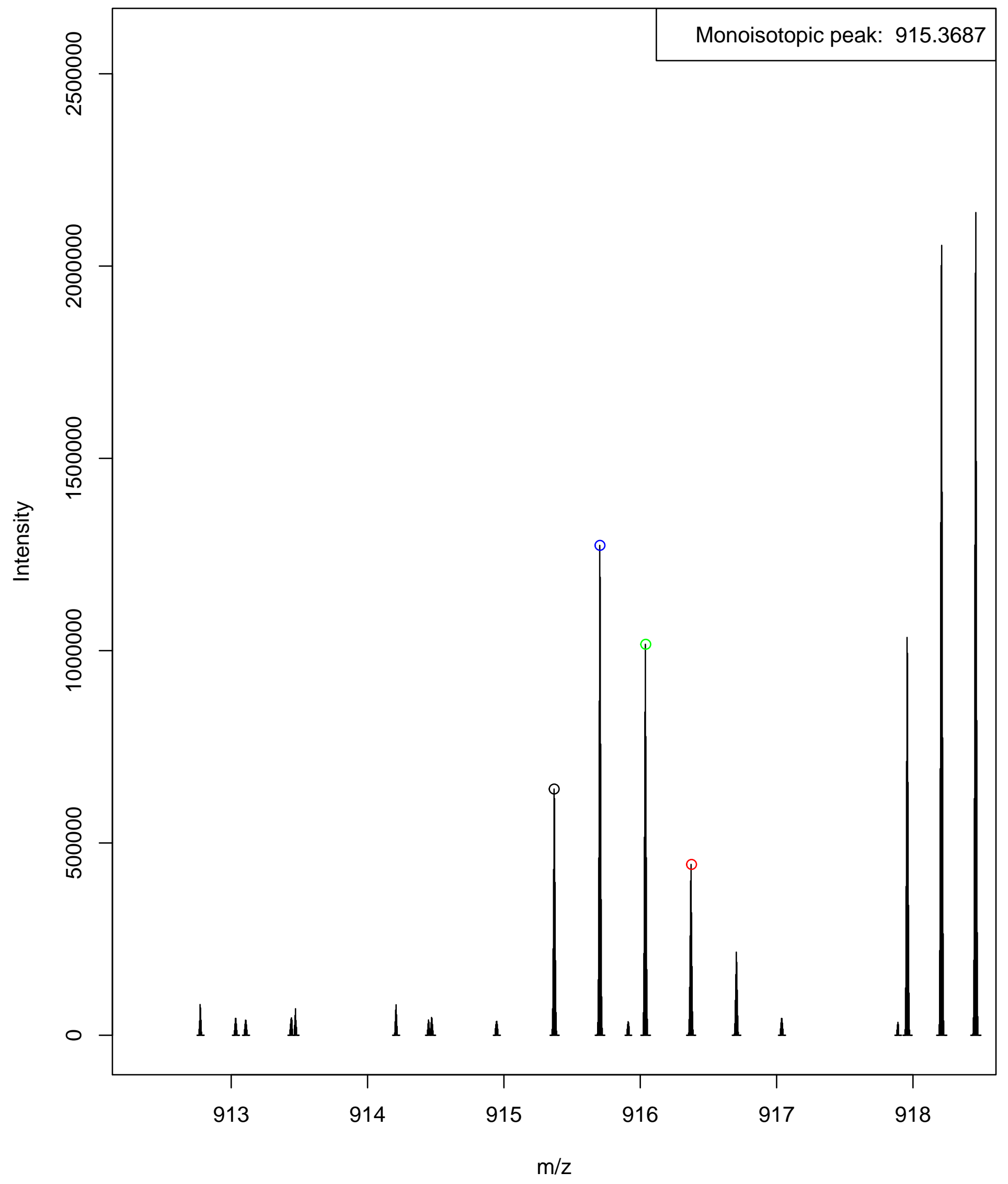


XIC of MS1

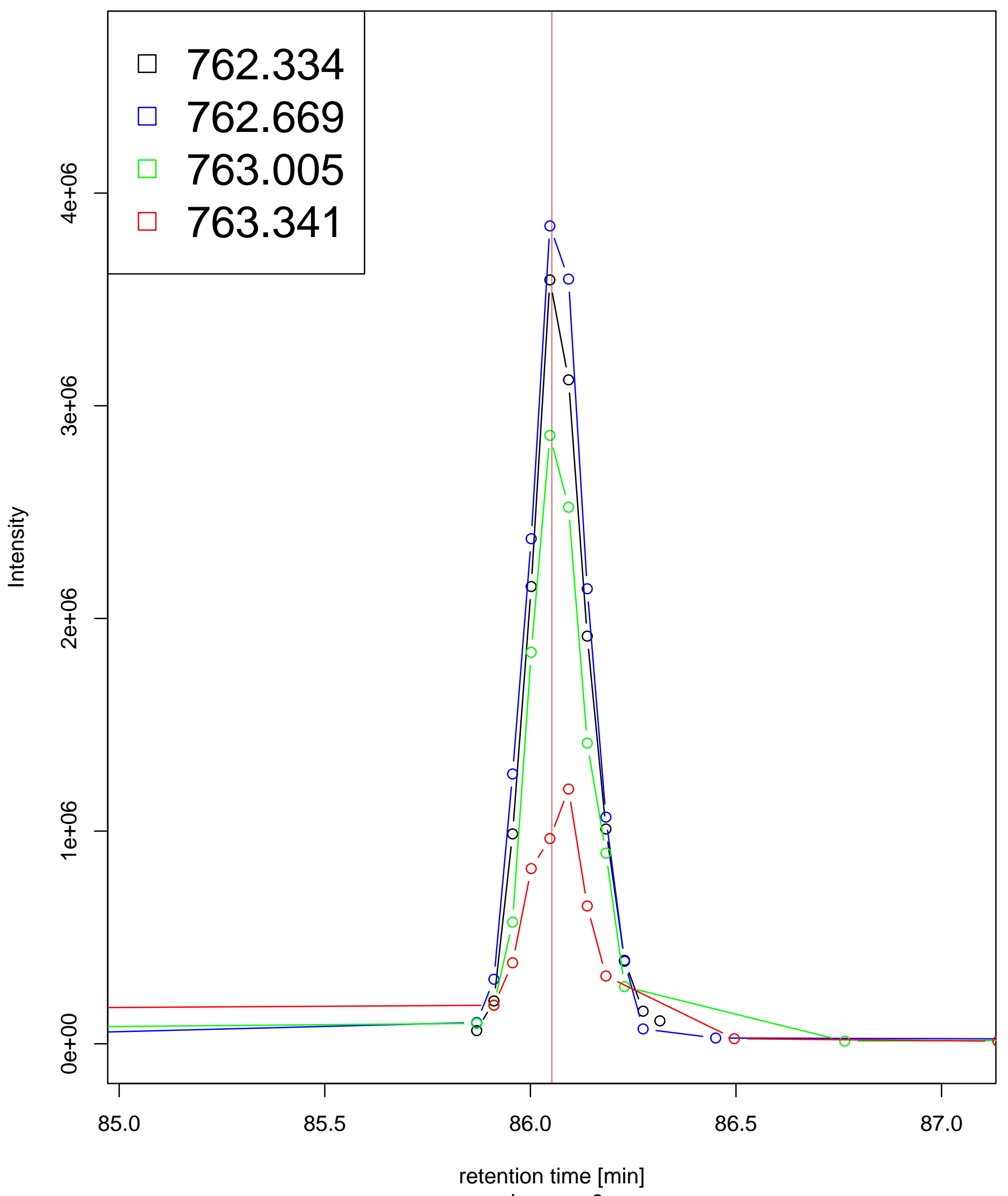

MS1 of ScanNo:16505

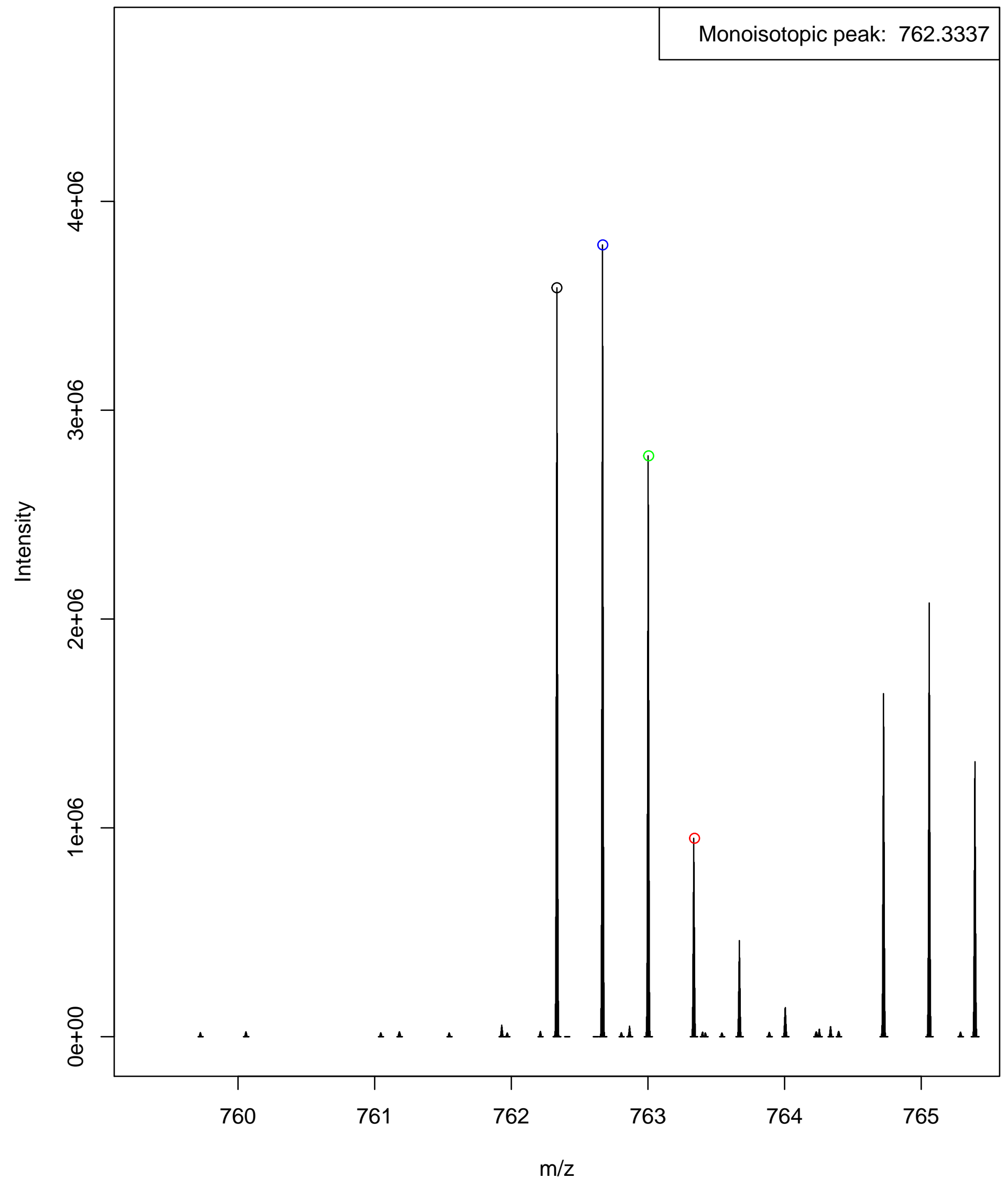

charge $=3$ 
XIC of MS1

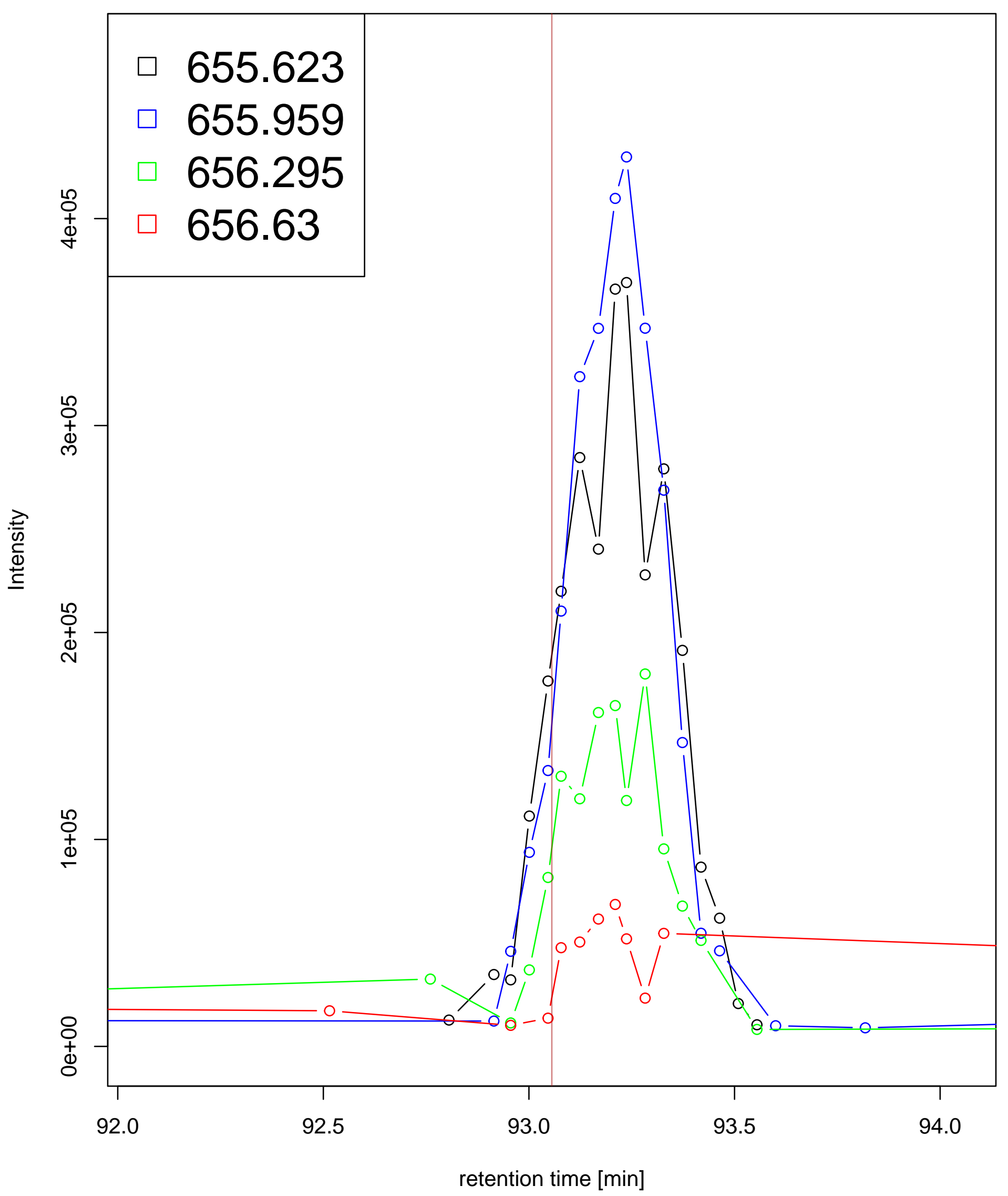

MS1 of ScanNo:18037

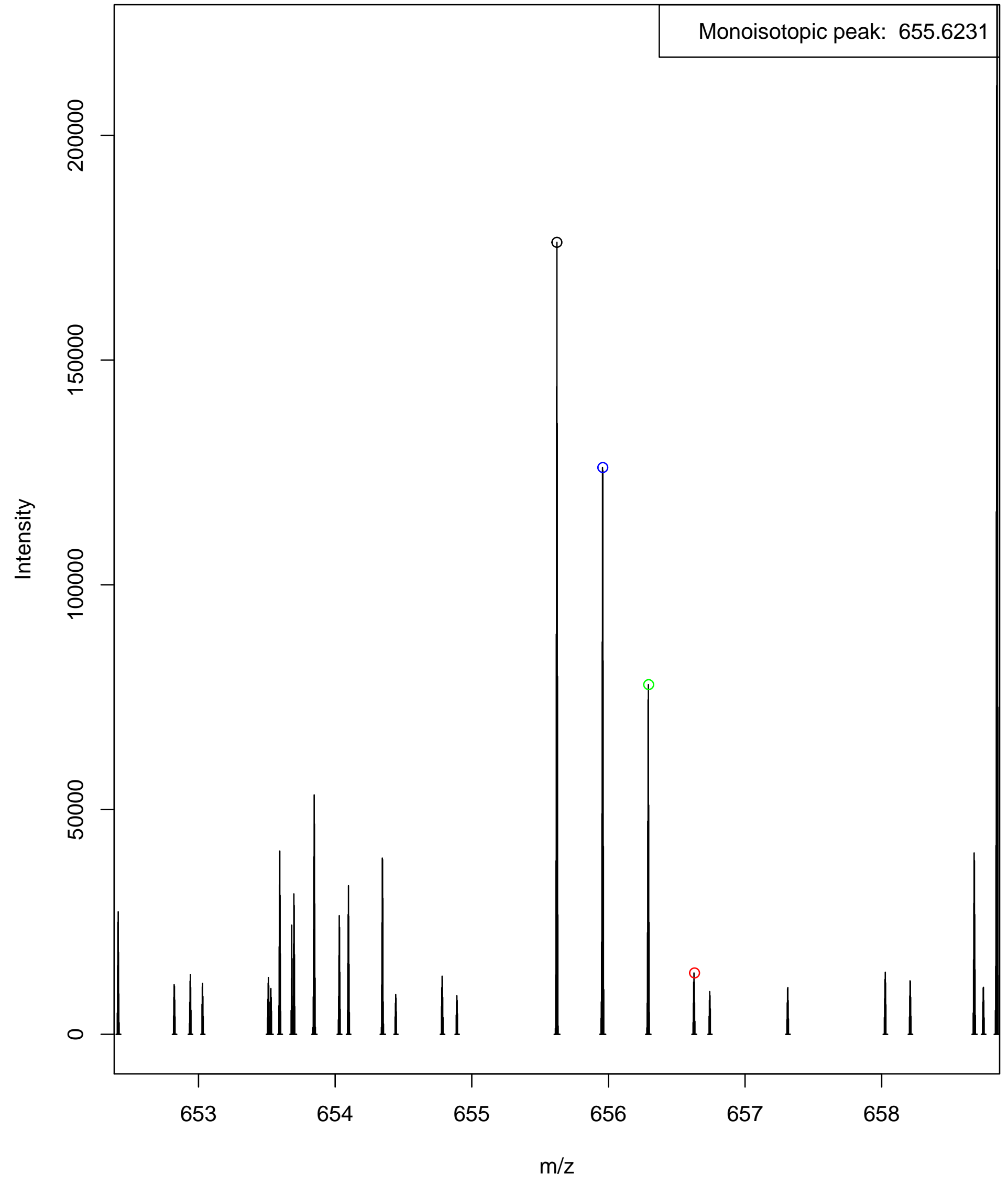

charge $=3$ 